REVUE FRANCAISE DE GEOTECHNTUE

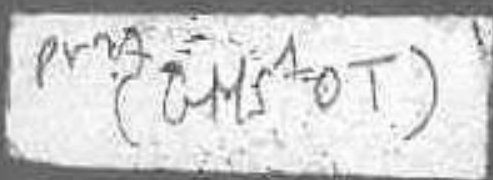

\title{
ETUDE GEOTECHINIQUE
}

ET

\section{RECONNAISSANCE DES SOLS}

Projet de DTU

(N.22)

Dresses 
Axisplsoly

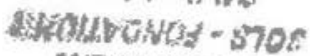

HNEKSLHVESO

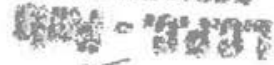

\section{ETUDE GEOTECHNIQUE}

\section{ET}

\section{RECONNAISSANCE DES SOLS}

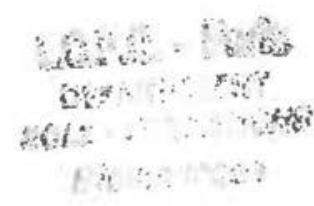




\section{Revue Française de Géotechnique \\ 4 numéros par an}

Editeur : Association Amicale des Ingénieurs Anciens Elèves de l'Ecole Nationale des Ponts et Chaussées

28 rue des Saints-Pères 75007 Paris Tél. 2603413 et 2601480

Directeur de la Publication : P. Habib

Président du Comité de Direction : A. Pasquet

Comité de Direction : P. Habib - P. Duffaut - C. Bordet - B. Mandagaran

Directeur du Comité de Rédaction : P. Londe

Comité de Rédaction :

E. Absi - F. Bonnechère - J. Goguel - J. Kérisel - P. La Rochelle

G. L'Hériteau - M. Panet - M. Rat - J. Salençon - F. Schlosser

Secrétaire : B. Mandagaran

Abonnement annuel : France : $350 \mathrm{FF}$ - étranger : $350 \mathrm{FF}$

Tous droits de reproduction, traduction, adaptation, totales ou partielies, sous quelque forme que ce soit, expressément réservés.

Les articles figurant au sommaire sont publiés sous I'entière responsabilité de leurs auteurs. 


\section{sommaire}

PREFACE Louis Parez 7

CHAPITRE I PRESCRIPTIONS GENERALES 11

CHAPITRE II METHODES DE SONDAGES

PAR FORAGES MECANIQUES

ET PRELEVEMENTS

CHAPITRE II LES SONDAGES ET LES ESSAIS "IN SITU»

A Standard penetration test 37

B Sondage au pénétromètre statique 41

C Sondage au pénétromètre dynamique 45

D Sondage pressiométrique normal $\quad 49$

E Sondage pressiométrique au pressiomètre autoforeur $\quad 59$

F L'essai au scissomètre $\quad 65$

G Les essais à la plaque $\quad 69$

H Les essais d'eau dans les sondages $\quad 83$

CHAPITRE IV LES METHODES GEOPHYSIQUES

ET LES ESSAIS DYNAMIQUES «IN SITU» 109

A Les méthodes géophysiques 111

B Les essais dynamiques «in situ» 117

CHAPITRE V LES ANALYSES

ET LES ESSAIS EN LABORATOIRE 119

A Essais d'identification des sols 121

$\begin{array}{ll}\text { B Essais de résistance mécanique } & 129\end{array}$

C Essais de compressibilité 135

D Essais de compactage $\quad 141$

E Essai de poinçonnement C8R 145

$\begin{array}{ll}F \quad \text { Essais cycliques et dynamiques } & 149\end{array}$

G Essais spécifiques sur les matériaux rocheux 151 


\section{préface}

Cet ouvrage n'est pas un Document Technique Unifié, mais, comme l'indique le titre choisi par ses auteurs, il rassemble plusieurs documents de travail élaborés par le groupe de spécialistes chargés de la rédaction d'un projet de modification du DTU 11-1 paru en décembre 1968 sous le titre "Travaux de sondage des sols de fondation».

En attendant la mise au point définitive de ce projet, l'édition de ces documents de travail par les Presses de I'Ecole Nationale des Ponts et Chaussées va donner aux Maîtres d'ouvrage, aux Maîtres d'œuvre et aux Concepteurs, l'opportunité de mieux apprécier la diversité des moyens actuellement mis à leur disposition pour parvenir à une meilleure définition des projets de fondation.

Les spécialistes y trouveront matière à réflexion sur le contenu et l'articulation de leurs interventions et sur la standardisation d'un certain nombre d'opérations et d'essais pour lesquels les «modes opératoires», déjà codifiés dans nombre de pays étrangers mais non encore rassemblés dans un seul document en France, feront l'objet, ici, de procédures suffisamment précises pour que l'on puisse, sans ambiguïté, bien identifier les résultats obtenus et les comparer entre eux.

A l'usage, de tels documents provoqueront sans doute quelques réactions dont les auteurs ne manqueront pas de tenir compte dans leurs travaux en cours, tant au niveau de l'achèvement de leur projet qu'au niveau des futures remises à jour.

Ceci n'est d'ailleurs pas le moindre intérêt d'une telle publication. 


\section{comité de rédaction}

\section{du projet de DTU}

Messieurs :

Michel ADAM

Directeur de la Réglementation à I'UTI

Samuel AMAR

Laboratoire Central des Ponts et Chaussées

Jean-Pierre BERGIN

Division Sols et Fondations du Bureau Véritas

Michel BOUCHERIE

Département Sols et Fondations de SOCOTEC

Jean-Raymond BRULOIS

Société Bachy

Maurice CASSAN ${ }^{*}$

Fondasol

Etienne de CAZENOVE

Société Solétanche

Robert CHADEISSON

Société Solétanche

Jean CLAUZON

Union Nationale de la Maçonnerie

Jean-Pierre COLLIN

CATED

Jean-Pierre DAUVISIS

Société ESF

Maurice FARHI

Société Mécasol

Michel GAMBIN

Société Techniques Louis Ménard

William GERY

Société de Conștruction Géry-Dutheil

Henri GONIN

Société Simecsol

Pierre HABIB

Laboratoire de Mécanique des Solides

(EP, ENSMP, ENPC)

André ISNARD

Division Sols et Fondations du Bureau Véritas

Jean LEBLANC

Société Etudes Pressiométriques Louis Ménard

Marc PANET

Laboratoire Central des Ponts et Chaussées

Louis PAREZ

Société Sol-Essais

Paul PFISTER

Société Sol-Expert International

Gérard PHILIPPONNAT

Service Géotechnique du CEBTP

Henri SORIA

Département des sols et Fondations du CEP 
chapitre I

\section{prescriptions générales}




\section{Objet}

Le présent cahier des charges a pour objet de définir les conditions techniques dans lesquelles doivent être conduites l'étude géotechnique relative à un projet de construction et corollairement la reconnaissance détaillée des sols de fondation nécessaire pour l'élaboration de cette étude.

\section{Domaine d'application}

Le présent cahier des charges est applicable aux études géotechniques et travaux de reconnaissance des sites de construction quelle que soit la destination de ces constructions.

\section{Définitions}

Cette étude confiée à l'ingénieur géotechnicien peut se dérouler en plusieurs étapes selon l'évolution des études du projet de construction envisagé. II est d'ailleurs utile qu'elle soit engagée dès le choix du site afin d'aider le maître d'cuuvre à la conception de son plan masse. II est recommandé de la poursuivre au moins jusqu'à la réalisation des fondations pour verifier la conformité des hypothèses et modèles de calcul pris en compte avec les résultats de l'observation directe en grand des sols mis à jour en cours de travaux.

\subsection{L'étude géotechnique}

L'étude géotechnique se rapporte aux sols et aux eaux souterraines en tant qu'éléments intervenant dans la stabilité et le bon comportement des constructions provisoires ou définitives.

Elle est destinée à fournir au maître d'œuvre les données relatives au comportement du sol qui lui sont nécessaires pour la conception et la construction des ouvrages et celles relatives à leur incidence sur l'environnement. 
Selon le programme de l'étude géotechnique, la reconnaissance des sols peut comporter plusieurs étapes et être modifiée en cours d'exécution après accord entre les parties.

Les qualifications $O P Q I B /$ correspondantes sont les qualifications $A 111$ ou A 121.

Les qualifications OPQIBI correspondantes sont les qualifications A 133 et A 134.

La qualification $O P Q I B I$ correspondante est la qualification A 131.

\subsection{La reconnaissance des sols}

Cette opération a pour objet la mise en œuvre des moyens nécessaires à une connaissance suffisante des divers éléments et formations composant le volume des sols concernés par le projet.

\subsection{L'ingénieur géotechnicien}

L'ingénieur géotechnicien est un ingénieur qualifié spécialisé en mécanique des sols et en conception des travaux de fondation.

II est chargé par le maître d'œuvre, pour le compte du maître d'ouvrage, d'une mission de conseil auprès du maître d'cuvre et des concepteurs pour :

- la définition du ou des programmes de reconnaissance,

- le contrôle de leur exécution,

- l'interprétation des résultats,

- la conception et le mode de réalisation des fondations adaptées à la structure et à la destination du ou des ouvrages à constrüire,

- I'étude et I'incidence des travaux à I'intérieur du périmètre géotechnique de protection.

\subsection{Le sondeur}

Le sondeur est l'exécutant qualifié de la campagne de reconnaissance et, à ce titre, il est responsable de la mise en cuvre des moyens nécessaires à l'accomplissement de cette reconnaissance telie que définie par l'ingénieur géotechnicien.

\subsection{Le laboratoire}

On désigne sous ce terme un laboratoire qualifié en mécanique des sols choisi par le maître d'ouvrage en accord avec l'ingénieur géotechnicien pour effectuer les essais demandés par ce dernier sur les échantillons de sol prélevés en cours de reconnaissance.

\subsection{Le périmètre géotechnique de protection (PGP)}

Le périmètre géotechnique de protection est la frontière de la zone dans laquelle :

- la bonne tenue du sol et des ouvrages existants d'une part,

- la bonne tenue des ouvrages à construire pendant et après les travaux d'autre part,

peuvent avoir des interactions qui doivent être étudiées. 


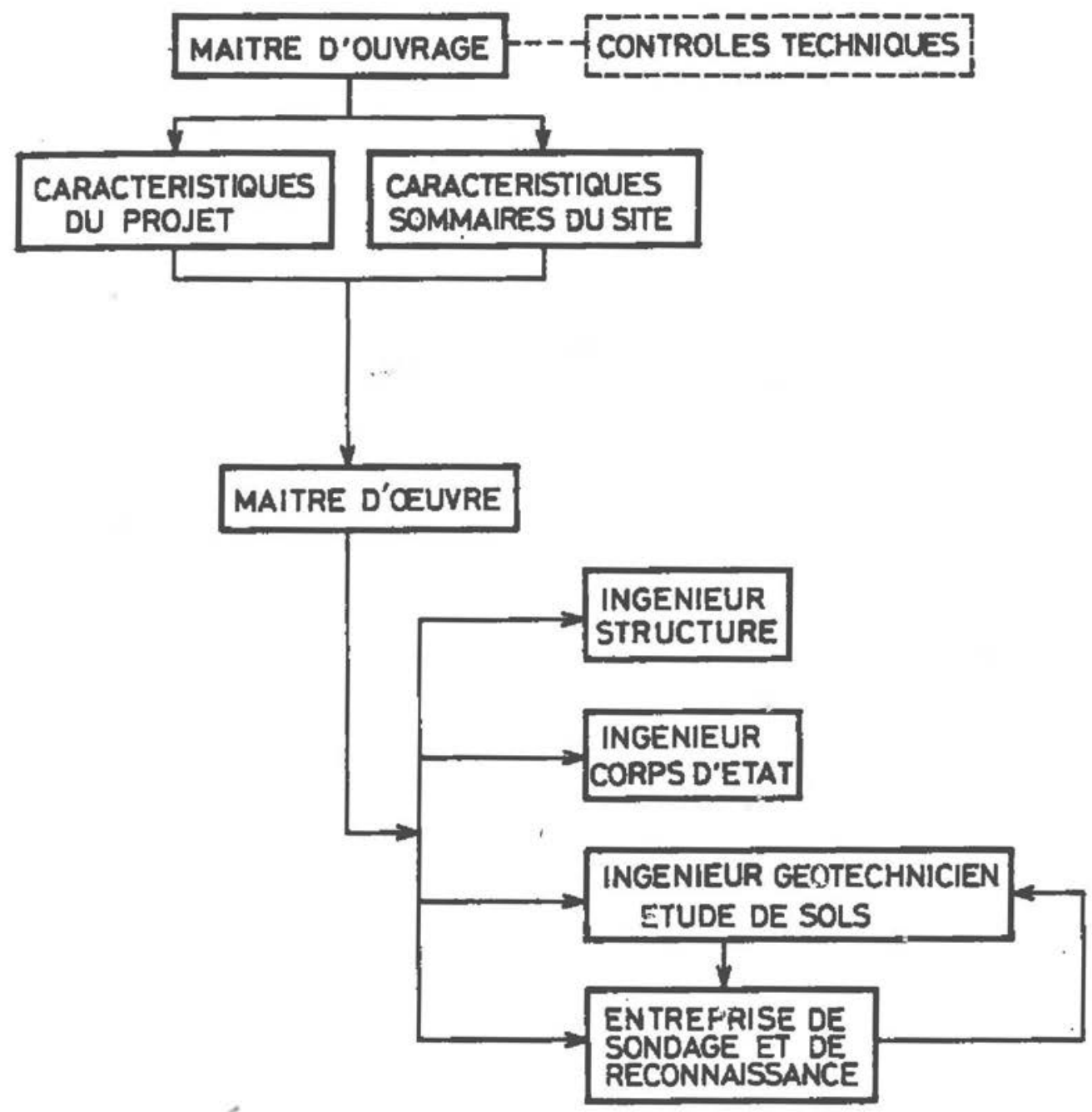


Les organismes officiels pouvant étre utilement consultés sont généralement les suivants :

- Directions Départementales de I'Equipement et de l'Agriculture,

- Services des Mines et de I'Inspection des Carrières,

- Service de la Protection Civile.

Les documents à consulter sont :

- les cartes topographiques at les photos aériennes IGN,

- les cartes géologiques,

- les cartes spécifiques : carrières, zermos, zonage sismique, etc.

On entend par "sondage» toute opération effectuée à la surface du sol et au sein du sous-sol pour permettre la reconnaissance des élements et formations constituant le sous-sol.

Le nombre de ces sondages est fonction :

- de l'étendue du périmètre géotechnique de protection et de l'hétérogénéité du sous-sol,

- de la sensibilité des structures à construire aux tassements différentiels.

L'interprétation correcte des résultats des essais in situ implique généralement l'examen approfondi des resultats d'un forage d'étalonnage assorti d'examens appropries pratiqués sur des échantillons représentatifs des formations sous-jacentes.

Dans le cas de fondations sur pieux, le DTU 13-2 "Fondations Profondes》 précise que cette profondeur doit dé-

\section{Le contenu de l'étude géotechnique}

\subsection{Enquête préalable}

Avant la mise en cauvre de la reconnaissance des sols proprement dite, il convient de procéder à une enquête préalable comprenant :

1) I'examen des caractéristiques principales du projet,

2) une visite détaillée des lieux,

3) la consultation des personnes et des documents susceptibles de fournir des informations sur le site, son environnement et sur le mode de fondation des ouvrages existants situés à proximité,

4) la rédaction d'une note de synthèse comprenant la définition d'un programme de reconnaissance adapté au site et au projet.

\subsection{Reconnaissance des sols de fondation}

La reconnaissance des sols de fondation dont le programme est défini par l'ingénieur géotechnicien doit comporter un certain nombre de sondages destinés à préciser sans ambiguïté :

- la position dans l'espace des divers éléments et formations constituant le sous-sol,

- I'identification géologique et physico-mécanique des sols soumis au champ de contraintes engendré par la construction à l'intérieur du périmètre géotechnique de protection.

Ces sondages comprennent des forages, des essais in situ et des prélèvements d'échantillons pour examens ou analyses en laboratoire.

La profondeur des sondages doit être supérieure à la profondeur des sols sur lesquels les fondations engendrent encore des efforts susceptibles de provoquer à 
passer d'au moins 7 diamètres lavec un minimum de $5 \mathrm{~m}$ l la cote prévue pour la pointe des pieux. La prise en compte des effets provoqués par des groupes de pieux peut conduire à augmenter cette profondeur bien audelà de la recommandation figurant ci-dessus. terme des déformations préjudiciabies à la bonne tenue de la construction.

Indépendamment des risques d'existence d'accidents naturels ou artificiels (cavités, zones décomprimées, effondrées ou remblayées, etc.l, cette profondeur doit être supérieure à celle des surfaces de glissement de masse les plus probables qui pourraient se manifester du fait des modifications temporaires ou définitives apportées par les tràvaux à la topographie et aux conditions hydrauliques naturelles du site.

4.3 Anialyse et synthèse de l'enquête préalable et de la reconnaissance

L'anaiyse des éléments recueillis au cours de l'enquê. te préalable et de la reconnaissance des sols conduit à une synthèse générale comportant :

1) des coupes et profils géologiques et géotechniques corrélant les résultats des sondages et essais et définissant la position dans l'espace et les dimensions des divers éléments et formations reconnus,

2) les diverses caractéristiques des sols nécessaires à I'élaboration du projet et à retenir en vue des calculs,

3) le régime des différentes circulations et nappes d'eau,

4) I'étude de la sensibilité au gel des sols de surface,

5) I'agressivité des sols et des eaux à l'égard des matériaux de construction prévus,

6) s'il y a lieu, l'étude du comportement des sols à l'égard des sollicitations dynamiques, (séismes, machines vibrantes, etc.).

4.4 Interprétation en vue du projet et de sa réalisation

L'interprétation porte sur les points suivants :

1) exécution des terrassements,

2) stabilité des talus provisoires ou définitifs et stabilité des soutènements,

3) drainage, rabattement de nappe et étanchéité des ouvrages enterrés,

4) modes de fondation des constructions :

- types et niveaux de fondations possibies,

- contraintes admissibles conseillées.

- évaluation approximative des tassements absolus et différentiels,

5) d'une façon générale, tous les points concernant I'interaction sol-structure. 
chapitre II

méthodes de sondages

par forages mécaniques

et prélèvements 


\section{Classification générale des forages}

Les forages peuvent être classés en trois catégories selon la méthode utilisée :

- les forages carottés,

- les forages semi-destructifs,

- les forages destructifs.

Le choix de cette méthode est conditionné par la nature des formations que l'on doit reconnaître ainsi que par le but recherché.

\section{Exécution des forages}

\subsection{Maintien des parois}

La méthode de maintien des parois doit prévenir tout éboulement.

\subsection{Forages en carottage continu}

\subsubsection{Définition}

Les forages carottés sont réalisés en découpant le terrain par rotation ou par poinçonnement à l'aide d'un tube creux appelé carottier muni à sa base d'une couronne ou d'une trousse coupante.

\subsubsection{Carottiers}

On distingue les carottiers rotatifs et les carottiers poinçonneurs.

Les carottiers rotatifs peuvent être de deux types : simples ou doubles avec ou sans gaine amovible. 
Les carottiers simples peuvent être parfois employés à sec dans les sols meubles s'ils permettent une récupération supérieure à $60 \%$.

Bentonite, amidon, etc.

Les deux premiers modes ne sont pas recommandés dans les sols à granulométrie grossière.

En revanche, le vibrofonçage peut être utilisé dans ces sols.

Si les gros eléments d'un terrain pulvérulent nécessitent pour leur traversée l'emploi du trépan, la technique du
L'usage des carottiers simples avec fluide de forage doit être limité aux reconnaissances sommaires en rocher sain. Il est interdit dans les terrains meubles, dans le rochér altéré et, d'une façon générale, dans tous les sols susceptibles d'être désorganisés par les fluides de forage.

Les carottiers poinçonneurs doivent être munis d'un dispositif qui maintienne la carotte en place pendant la remontée de l'outil.

D'une façon générale, tous les carottiers, qu'ils soient rotatifs ou poinçonneurs, doivent permettre, si les documents du marché le précisent, le prélèvement d'échantillons intacts. Dans ce cas, les opérations doivent être conduites selon les modalités du paragraphe «Prélèvement d'́ćchantillons intacts».

\subsubsection{Forages rotatifs}

Les forages rotatifs ne peuvent être réalisés efficacement que dans les sols fins doués d'une certaine cohésion ou dans le rocher.

Dans les sols à forte cohésiọn, tels que le rocher, les marnes, les argiles raides et même moyennes, le fluide de forage peut être l'eau claire. Dans les argi- les molles, les sables argileux, les grés friables, le rocher altéré, il faut utiliser une boue de forage.

Toutefois, il convient de ne pas perdre de vue que les fluides de forage peuvent masquer les phénomènes hydrauliques.

\subsubsection{Forages par poinçonnement}

Les forages par poinçonnement peuvent être réalisés soit par vérinage, soit par battage, soit par vibrofonçage.

\subsection{Forages semi-destructifs}

\subsubsection{Définition}

On appelle semi-destructif un procédé qui ne permet pas le prélèvement d'échantillons intacts au sens de la mécanique des sols, mais qui permet d'extraire le sol d'une façon continue sous forme remaniée.

\subsubsection{Différents procédés de forages semi-destructifs}

\section{- Forages à la soupape}

Cette méthode ne doit être utilisée que dans les terrains pulvérulents. Le diamètre de l'outil doit être supérieur au diamètre des plus gros éléments du sol. 
forage à la soupape pour leur extraction devient une methode destructive (voir 2.4).

L'emploi des forages a la tarière mecanique n'exclut pas l'utilisation de la tarière à main lorsque la profondeur a atteindre et la consistance des sols s'y prêtent.

L'implantation des forages a la benne doit etre, dans la mesure du possible, située en dehors des zones de fondation.

\section{Domaines d'utilisation :}

Les forages destructifs peuvent stre utilisés dans les cas suivants :

- reconnaissances géologiques rapides, - forages pour essais géotechniques spéciaux lessais pressiométriques, essais d'eau, diagraphies),

- recherche des cavites,

- terrains où le carottage est difficile.

On maintient constantes, autant que possible, la pression de l'outil et la vitesse de rotation, sinon on en notera les variations. On note par ailleurs la pression du fluide de perforation.
- Fórages à la tarière mécanique

Ce type de forage est applicable aux terrains doués d'une certaine cohésion, tels qu'argile molle à moyenne, sable argileux, grave argileuse.

Dans ce dernier cas, le diamètre doit être choisi en fonction des dimensions des plus gros éléments.

- Forages à la benne preneuse

Leur utilisation est particulièrement bien adaptée à l'étude des formations alluvionnaires à granulométrie grossière mais il faut alors maintenir les parois du forage.

La méthode peut également être utilisée en terrains cohérents si leur consistance permet la pénétration de l'outil.

\subsection{Forages destructifs}

\subsubsection{Definitions}

Les forages destructifs sorit réalisés, en général, par battage, rotation ou par vibration d'un outil spécial, le trépan, qui désorganise le matériau. Un fluide, air, eau ou boue, injecté par le train de tige, permet de remonter les sédiments, et de les identifier. Dans le cas contraire, les sédiments sont récupérés à l'aide d'une soupape.

\subsubsection{Relevé des parametres de forage}

Lorsque le forage est réalisé, soit à l'air comprimé par vibration-percussion (wagon-drill), soit au tricône en rotation, on doit mesurer, de façon quasi-continue, la vitesse d'avancement de l'outil. Cette vitesse est évaluée soit directement à partir de repères tracés sur le train de tige, soit par enregistrement continu avec un dispositif approprié.

\subsection{Rebouchage des trous}

Sauf dispositions particulières prévues au marché, une fois le forage terminé, les trous doivent impérativement être rebouchés par le foreur. 
En particulier en terrain aquifère, on doit effectuer cette opération à l'aide d'un mélange argile-ciment afin de rétablir la continuité des couches imperméables traversées. Les mêmes précautions sont à prendre pour les forages réalisés sur des pentes afin d'éviter que les eaux de précipitation pénètrent dans le sol, ce qui peut compromettre la stabilité du versant.

\section{Echantillons remaniés}

\subsection{Prélèvement}

Avant tout prélèvement d'échantillons, il est procédé au nettoyage de la surface sur laquelle le prélèvement est prévu.

Si les échantillons remaniés sont destinés à des essais simples d'identification géotechnique en laboratoire, la méthode de prélèvement doit permettre d'obtenir un échantillon de granulométrie représentative du terrain.

Les quantités prélevées doivent permettre l'exécution de l'ensemble des essais de laboratoire pour lesquels ils sont prévus.

\subsection{Conditionnement et étiquetage}

Aussitôt les prélèvements effectués, ceux-ci sont placés dans des emballages étanches.

A chaque échantillon est jointe une fiche sur laquelle sont portées d'une façon inaltérable les indications suivantes :

- nom du chantier,

- numéro du forage,

- date de prélèvement,

- niveaux ou profondeurs entre lesquels l'échantilIon a été prélevé.

- nature approximative du sol.

\subsection{Conservation et transport}

Les manutentions et le stockage doivent être conduits de façon à ne pas détériorer les emballages et les étiquetages.

\section{Echantillons intacts}

\subsection{Prélèvement}

\subsubsection{Précautions à prendre avant prélèvement}

L'action des outils de terrassement ou de forage et celle du tubage amènent un remaniement sensible des sols sur une certaine épaisseur qu'il y a tieu d'éliminer. 
- La gaine évito la manipulation directe de l'échantillon,

- le prélevement du sol se fait par poinconnement. Si la consistance du sol ne le permet pas, le prélèvement est fait à rotation.

Dans les sols très mous, il est recommandé de procéder à l'enfoncement du carottier par pression.

Si l'enfoncement se fait par pression, il est effectué en continu, sans aucune interruption ni mise en rotation du carottier.

La vitesse de fonçage est réglée en fonction de la consistance du sol et est généralement comprise entre 15 et $30 \mathrm{~cm} / \mathrm{seconde}$.

Les diametres adoptes pour les carottes sont en général compris entre 50 et $100 \mathrm{~mm}$. Chaque fois que cela est possible, il est conseille de choisir des diamètres supérieurs à $70 \mathrm{~mm}$, les carottes offrant alors de plus grandes facilités pour l'exécution des essais de laboratoire. Par ailleurs, toute chose égale, le remaniement latéral super. ficiel du au prélèvement est proportionnellement moins important pour les carottes de grand diamètre.

Le coefficient d'entrée ou jeu intévieur du carottier défini à l'annexe contrôle le frottement de la carotte à I'intérieur de l'appareil de prélèvement.

L'indice de surface ou coefficient de paroi du carottier défini ci-apres represente le rapport du volume de terrain déplacé par la trousse coupante au volume de l'échantillon.

\subsubsection{Exécution du prélèvement}

Compte tenu de la nature présumée des terrains, les appareils et les méthodes de prélèvement sont proposés par. l'entreprise de forage et approuvés par le maître d'ouvrage ou le géotechnicien qu'il aura délégué.

L'emploi du carottier rotatif simple est interdit, seul le carottier rotatif double est autorisé.

Dans le cas de sols cohérents allant de I'argile consistante au rocher dur, on utilise un carottier rotatif double.

Dans le cas de sols de consistance moyenne, le carottier doit comporter une gaine de conditionnement.

Dans le cas des sols mous, on ne doit utiliser que des carottiers munis d'une gaine ou faisant office de gaine enfoncés par poinçonnement.

4.1.2.1 Le type et les dimensions des appareils sont déterminés en fonction de la nature du sol et du type d'essai de laboratoire à réaliser.

4.1.2.2 Les caractéristiques géométriques des carottiers poinçonneurs destinés aux sols mous doivent être telles que le coefficient d'entrée ou jeu intérieur $\mathrm{Ci}$ soit compris entre 0,5 et $1,5 \%$ et l'indice de surface, ou coefficient de paroi $\mathrm{Ca}$, soit inférieur à $15 \%$.

Dans le cas des sols de consistance moyenne, ces coefficients deviennent

$\mathrm{Ci} \leqslant 4,5 \%$

$\mathrm{Ca} \leqslant 25 \%$ 


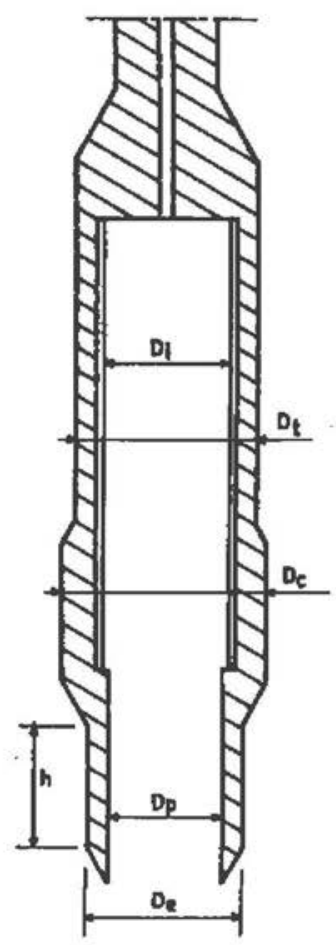

CARACTERISTIOUES GEOMETRIQUES DES CAROTTIERS PONCONEURS

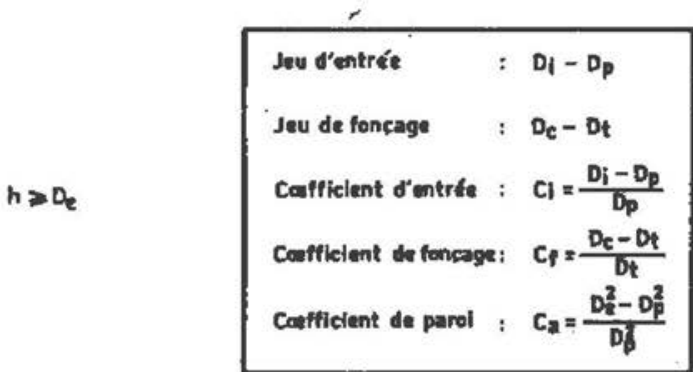

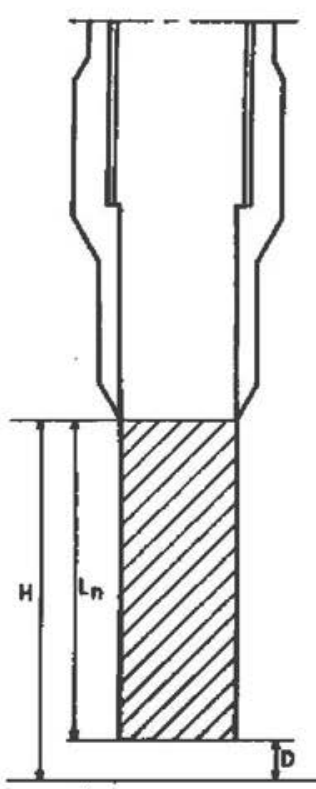

\section{DEFINITION DU POURCENTAGE ET DE L'INDICE DE CAROTTAGE.}

H ; Pénclration du carottier.

L ; Longueur de la caratte dans le carottier. Lnt longueur de la carottic dins le sol.

D : Déflexion des couches sous la trousse coupante.

Pourcentage de carotage : $\frac{1}{H} \times 100 \%$ Indier de carottage : $\frac{\Delta i}{\Delta H}$

AL i Longueur de la carotte dans le earottier correspondant à un enfoncement $\mathrm{AH}$. 
La longueur utile des carottes est genéralement comprise entre $0,4 \mathrm{~m}$ et 1 metre.

La partie coupante de la trousse est surmontee d'une partie cylindrique de hauteur "h" au moins égale à son diamétre extérieur a Dey.
4,1.2.3 La pénétration totale du carottier ne doit, en aucun cas, être supérieure à la longueur intérieure du carottier.

La mise en flexion ou en vibration du train de tige est intẹrdite pendant le prélèvement.

\subsection{Conditionnement et étiquetage}

Lorsque lè carottier comporte une gaine, on procède aux opérations suivantes :

- démontage du carottier,

- mise en place de bouchons étanches,

- étiquetage.

Dans le cas contraire qui ne doit être utilisé que pour des sols à très forte cohésion ou pour des roches, on place les échantilions recueillis dans une gaine de longueur et de diametre adaptés à la taille des échantillons. On procède ensuite à la mise en place des bouchons étanches et à l'étiquetage comme précédemment.

Une étiquette doit être collée sur la gaine, elle comporte d'une manière inaltérable les indications suivantes :

- référence du chantier,

- nom de l'entreprise de sondage,

- numéro de sondage,

- date du prélèvement,

- niveaux inférieur et supérieur du prélèvement,

- type de carottier utilisé - nombre de coups de mouton si le prélèvement a été fait par battage, - nature approximative du sol visible aux extrémités de l'échantilion.

D'autre part, le haut et le bas du prélèvement doivent être indiqués sur la gaine.

\subsection{Conservation et transport}

Sur le chantier, la conservation se fera dans un local à l'abri du gel mais également des températures trop élevées et des vibrations.

Les échantillons intacts sont acheminés rapidement au laboratoire:

Le transport des échantillons doit se faire en plaçant ceux-ci dans des caisses bourrées avec une matière amortissante (paille, copeaux de bois, mousses synthétiques...). II y a lieu de s'assurer qu'il n'y a aucun risque de gel pendant le transport et, en particulier, le transport en avion. 
On utilise des couvercles à glissière ou à charnière.

I/ est preférable de porter les inscriptions sur un c6té de la caisse.

Dans le cas de forages semi-destructifs ou destructifs, il n'est pas indispensable de mettre en caisse la totalite des materiaux extraits.

II suffit, à chaque changement de terrain, de choisir un echantillon d'apres son caractère représentatif et de le mettre, soit directement dans la caisse si la quantite de matière le permet, soit dans un sachet transparent que I'on place dans la caisse. A chaque sachet, doit être fixée une étiquette (voir article 3.2).

Ce carnet est tenu sur le chantier à la disposition du maitre de l'ouvrage ou de ses représentants.
5 Conservation et présentation des sols extraits

Les sols extraits sont soigneusement conservés dans des caisses compartimentées protégées par un couvercle.

Ces caisses doivent être suffisamment rigides et résistantes pour supporter.sans dommage les manutentions.

Sur chaque caisse, doivent être notés de façon inaltérable :

- le nom du chantier,

- le numéro du forage,

- le numéro d'ordre de la caisse pour le forage considéré,

- les profondeurs extrêmes des terrains contenus dans la caisse.

Dans le cas de forages carottés, la totalité des carottes est placée dans les caisses et, pour chaque passe de carottage, on note les profondeurs sur les cloisons longitudinales séparant les différents compartiments.

De plus, les différentes passes de caroțtage sont séparées par une cale transversale.

A l'emplacement de chaque échantillon envoyé au laboratoire, on met deux cales de séparation et on note sur la cloison longitudinale : "échantillon».

Pendant la durée de la campagne de reconnaissance, les caisses sont conservées à l'abri dans des conditions permettant d'éviter la dégradation, la destruction ou la disparition de leur contenu.

A l'issue de cette campagne, ces caisses sont remises aux bons soins du maître d'ouvrage.

\section{Carnet de forage}

Pour chaque forage, l'entrepreneur de forage tient à jour un carnet où sont consignées les observations sui- 
Les profondeurs sont mesurées à partir de la surface du terrain perfore $1 y$ compris an site aquatique).

On entend par "passe courte» des passes de l'ordre de $20 \mathrm{~cm}$.
On note en particulier les profondeurs où ont été décelés les éboulements accidentels de paroi, les chutes d'outils, les remontées de sol, etc., ainsi que l'importance de ces incidents. vantes faites pendant l'exécution du forage :

- la désignation du chantier,

- le nom de l'entreprise de forage,

- la numérotation du forage permettant de le repérer sur un plan de situation,

- son inclinaison par rapport à la verticale descendan-

te et son azimut,

- le norn du spécialiste responsable de la conduite de l'outillage,

- les dates de façon à faire apparaître quotidiennement les travaux effectués,

- les profondeurs,

- la qu les méthodes de perforation avec leur profondeur d'utilisation et la nature de l'outil utilisé,

- le ou les diamètres du trou,

- le ou les tubages avec leurs profondeurs respectives,

- l'indication du fluide de perforation.

- les indications nécessaires relatives aux carottes prélevées :

- la longueur de la passe de carottier,

. la longueur de carotte récupérée,

- la nature et la profondeur des différents essais réalisés "in situ»,

- dans le cas de forage destructif, les vitesses instantanées de perforation mesurées sur des passes courtes ou enregistrées,

- les profondeurs entre lesqueiles les échantillons ont été prélevés ainsi que le mode de prélèvement,

- la nature des terrains traversés estimée par le sondeur,

- toutes les observations concernant les arrivées d'eau et les pertes de fluide de perforation,

- la profondeur de l'eau ou du fluide de perforation dans le forage en début et fin de poste, - les incidents éventuels de forage.

\section{La coupe de forage}

Pour chaque forage, I'entrepreneur établit une coupe de forage qu'il remet au maître de l'ouvrage ou à ses représentants. Cette coupe de forage mentionne :

- la désignation du chantier,

- le nom et l'adresse de l'entreprise de forage,

- la numérotation du forage et la référence du plan de situation permettant de le repérer,

- en cas de forage non vertical, I'inclinaison par rapport à la verticale descendante et l'azimut, 
Les coordonnees du point de refférence fixe doivent être rapportées au système géodésique local officiellement en vigueur.

Les forages destructifs ne permettent pas l'identification géologique précise des dépôts et assises traversés.

Ce relevé peut figurer en annexe à la coupe de forage.

Leur confection exigeant des précautions exceptionnelles, les piézometres à prises de pression multiples sont à éviter.

A defaut d'autre méthode, la densité de fracturation est appréciée par 'le ROD qui s'exprime par la formule suivante :

$R O D(e n \%)=$

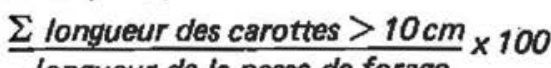

- la date du début et de la fin de l'exécution du forage.

La coupe de forage mentionne en particulier dans les colonnes spécifiques :

- le repérage en profondeur et en altitude par rapport à un point de référence fixe, des renseignements portés sur la coupe de forage,

- la description détaillée des terrains traversés,

- la représęntation symbolique des couches de terrain selon la grille annexée à ce chapitre,

- les attributions géologiques essentielles des dépôts et assises, cette attribution devant être faite, à la demande du maître de l'ouvrage ou de ses représentants, par un spécialiste,

- la ou les méthodes de perforation avec leur profondeur d'utilisation et la nature de l'outil utilisé,

- le ou les diamètres du trou,

- le ou les tubages avec leurs profondeurs et diamètres respectifs,

- I'indication du fluide de perforation,

- le pourcentage de récupération pour les forages carottés,

- les vitesses instantanées de perforation mesurées sur des passes courtes ou enregistrées pour les forages destructifs,

- l'indication des niveaux haut et bas des échantillons intacts prélevés et du mode de prélèvement utilisé,

- les observations concernant les arrivées d'eau et les pertes du fluide de perforation,

- le relevé du niveau de l'eau ou du fluide de perforation dans le forage, ainsi que la profondeur de la base du tubage et du fond du forage en débüt et en fin de journée ou de poste,

- les renseignements éventuels sur l'équipement piézométrique (diamètre du piézomètre - niveaux haut et bas de la crépine - dispositif d'étanchéité mesures datées du niveau hydrostatique, etc. $\}$,

- pour les forages en terrain rocheux, I'indication de la densité de fracturation,

- I'indication des niveaux des différents essais effectués en cours de forage (prélèvements d'eau, mesures de perméabilité, photographies, diagraphies, essais mécaniques in situ, etc.),

- I'indication des incidents de forage. 


\begin{tabular}{l|l|l}
\hline classe & $R Q D \%$ & description de la fracturation \\
\hline$R O D 1$ & $>90$ & densite de fracturation trés faible \\
$R O D 2$ & 90 d 75 & densite de fracturation faible \\
$R O D 3$ & 75 a 50 & densite de fracturation moyenne \\
$R O D 4$ & 50 d 25 & densite de fracturation forte \\
$R O D 5$ & $<25$ & densite de fracturation tres forte \\
\hline
\end{tabular}

Cette information peut être utilement complétte par l'estimation de l'indice de fracturation " $I_{f} \|$ correspondant à la longueur médiane des carottes prélevées sur une passe de carottage.

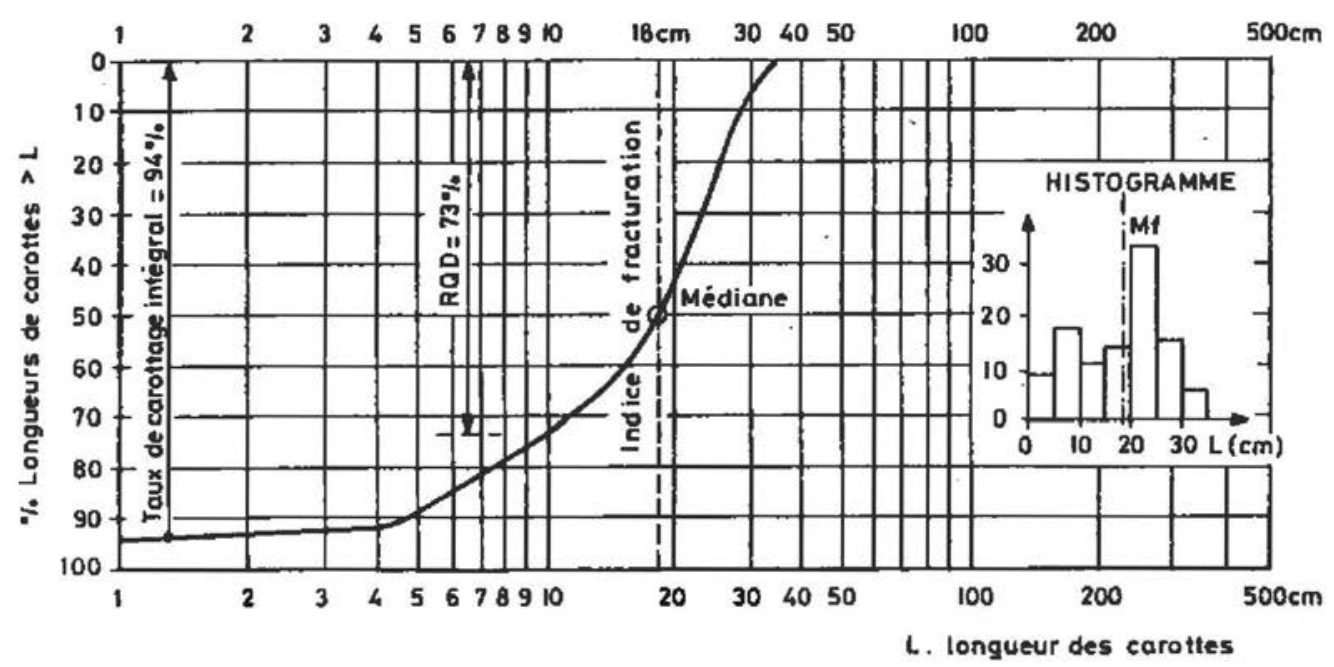




\section{TERRAINS D'APPORT - MACONNERIE}

\section{R Remblai}

\section{TERRAINS MEUBLES NON COHERENTS}

002000
0,00300
000

Sable grossier

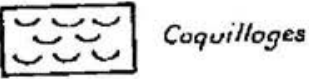

\section{TERRAINS MEUBLES COHERENTS}

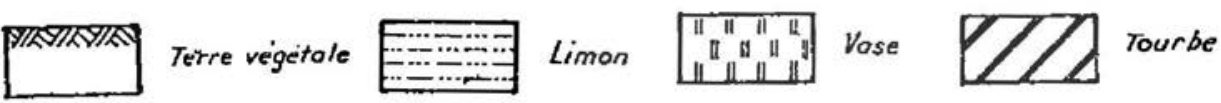

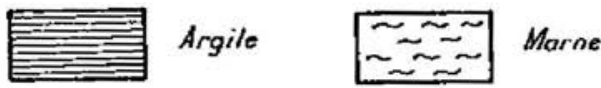

\section{ROCHES SEDIMENTAIRES}

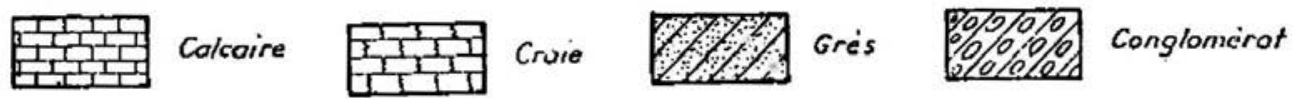

ROCHES VOLCANIQUES

$$
\begin{aligned}
& \text { Roches } \\
& \text { AA A A An geinéral }
\end{aligned}
$$

ROCHES METAMORPHIQUES

Roches

en genéral

\section{ROCHES CRISTALLINES}

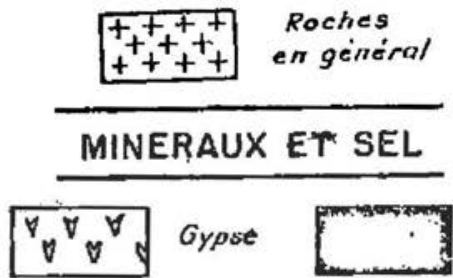

Charbon Liguite.

Grille de représentation symbolique des terrains 


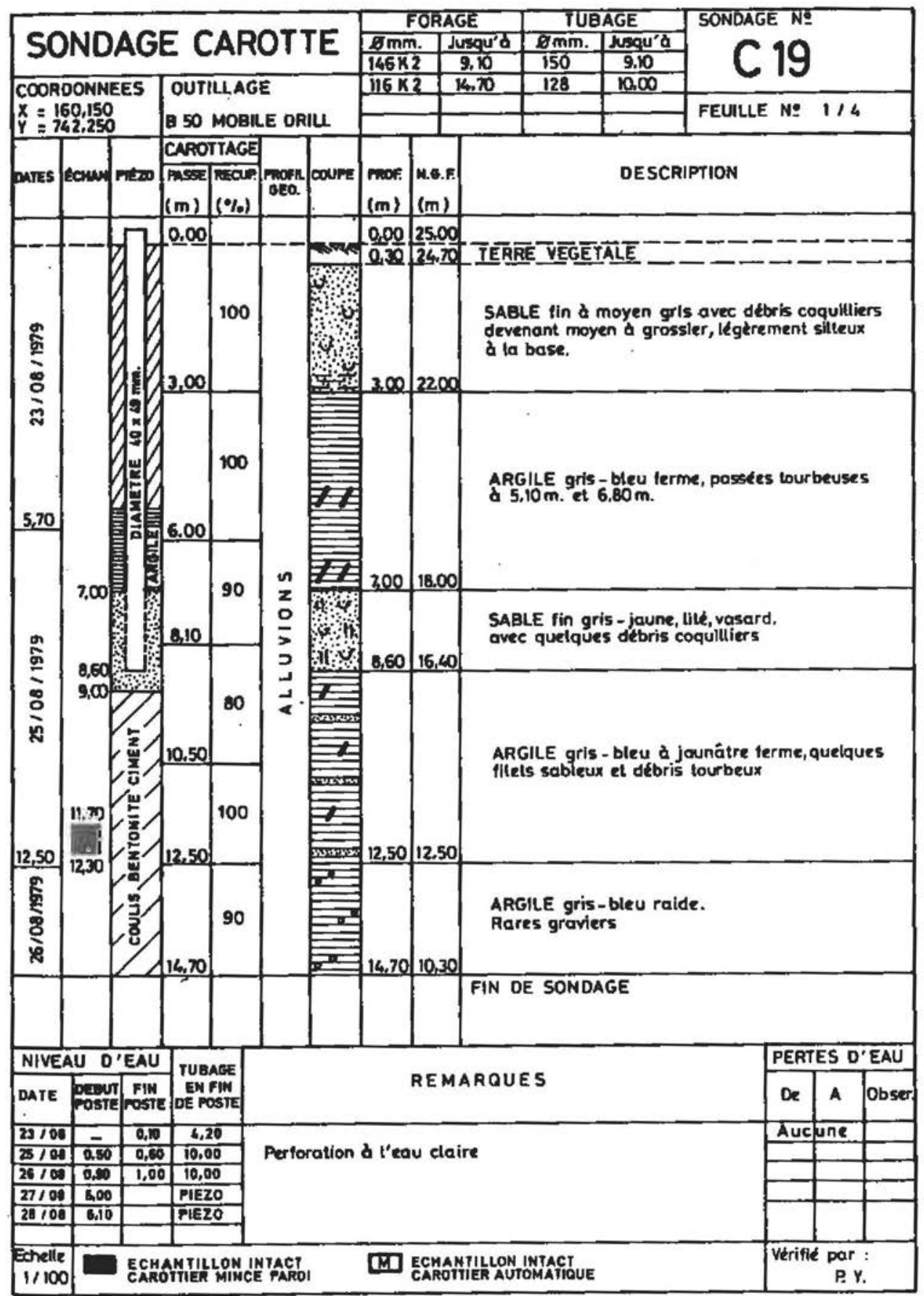

CHANTIER : SAINT NICOLAS _ Usine de sabots 
chapitre III

\section{les sondages et les essais "in situ"}




\section{A. standard penetration test}

Les intervalles seront de préférence choisis égaux à $1 \mathrm{~m}$.

Ce type d'essai est essentiellement adapté aux terrains à prédominance sableuse. L'interprétation ne peut valablement se reférer aux règles publiées par différents auteurs que si ces dernières se rapportent réellement à la valeur $N$ définie par la norme.

\section{Définitions}

1.1 L'essai SPT est un essai permettant de déterminer la résistance à la pénétration dans les terrains d'un tube échantillonneur fendu, enfoncé par battage dans un forage, et d'obtenir des échantilions remaniés du sol pour examen et identification. Cet essai se fait avec un matériel standard et suivant un processus normalisé. II doit être fait dans lẹs conditions fixées par la norme européenne en vigueur ci-après.

1.2 Un sondage SPT est un forage dans lequel les essais SPT sont effectués à des intervalles de pro: fondeur réguliers et compris entre $0,50 \mathrm{~m}$ au minimum et $1,50 \mathrm{~m}$ au maximum.

1.3 La valeur SPT «N» est une valeur caractérisant conventionnellement la résistance à la pénétration dans les terrains à prédominance sableuse dans les conditions suivantes :

- cette valeur résulte d'un essai SPT effectué selon les règles de la norme avec un matériel répondant aux critères qu'elle définit ;

- I'essai SPT correspondant a été effectué au fond d'un forage dans un terrain aussi peu remanié que possible, et notamment en évitant toute remontée ou éboulement de sol entre la fin de la perforation et le début de l'essai, dans les conditions suivantes : 
I/ est conseillé de faire apparaître le nombre de coups de mouton de l'essai préparatoire et les deux nombres obtenus au cours de l'essai proprement dit, par tranche de $15 \mathrm{~cm}$ d'enfoncement du carottier, dont la somme constitue la valeur SPT "N》.

Il est conseillé de reporter sur le graphique le ou les niveaux des nappes d'eau rencontrées au cours du forage, s'ils ont pu être relevés, ou d'indiquer dans un nota que ce renseignement n'est pas connu.

Dans le cas où les terrains sont saturés à faible profondeur, il est conseillé de faire, à côté du sondage SPT, un avant-
. Essal préparatoire de mise en place :

enfoncement du carottier de $0,15 \mathrm{~m}$ y compris la pénétration initiale sous le poids de l'échantillonneur et des tiges. Si cet enfoncement n'est pas obtenu après 50 coups de mouton, le battage de mise en place doit être arrêté.

\section{. Essai proprement dit :}

enfoncement de $0,30 \mathrm{~m}$ après la mise en place. $\mathrm{Si}$ la pénétration de $0,30 \mathrm{~m}$ n'est pas obtenue après 50 coups de mouton, l'essai est arrêté.

La vaieur SPT "N" est le nombre total de coups de mouton donnés pendant l'essai proprement dit ; elle est toujours inférieure ou égale à 50 . Dans le cas où elle est égale à 50, of doit noter l'enfoncement obtenu.

Le nombre de coups de l'essai préparatoire ainsi que les nombres de coups obtenus au cours de l'essai proprement dit pour obtenir les 15 premiers centimètres d'enfoncement et les 15 derniers, sont notés sur le chantier.

\section{Présentation des résultats}

\subsection{Sondages SPT - Graphique de pénétration}

Les résultats des essais SPT effectués dans un sondage SPT sont présentés sous forme de graphiques de pénétration, en fonction de la profondeur du début des essais, suivant une échelle arithmétique du nombre de coups de mouton donnés (allant de 0 à 50 ) :

- une première courbe, tracée en trait interrompu ou pointillé, donne la valeur des coups de mouton de I'essai préparatoire,

- une deuxième courbe, tracée en trait plein, donne les résultats de l'essai SPT lui-même,

- l'enfoncement obtenu pour les $\mathbf{5 0}$ derniers coups de mouton est indiqué s'il y a lieu.

2.2 Renseignements à porter en regard du graphique de pénétration

En regard du graphique de pénétration, on doit faire figurer :

- la coupe schématique des terrains traversés, avec la précision que permettent d'obtenir les renseignements recueillis,

- les dates auxquelles les essais ont été effectués.

- les tubages et modes de perforation utilisés,

- les profondeurs auxquelles ont été effectués les essais. 
trou jusqu'à la rencontre de l'eau pour noter de manière précise son niveau statique de surface au moment des essais.

Lorsque des essais d'identification ont été effectués au laboratoire sur les échantillons recueillis au cours des essais SPT, il est conseillé d'en faire figurer les resultats on regard du graphique de pénétration.

\subsection{Autres renseignements}

Le graphique de présentation des résultats doit comporțer le numéro d'identification du forage, les dates d'exécution, la cote de départ se référant à une altitude définie pour l'ensemble des sondages effectués sur un même site et éventuellement ses coordonnées on plan dans un système de référence précisé. 


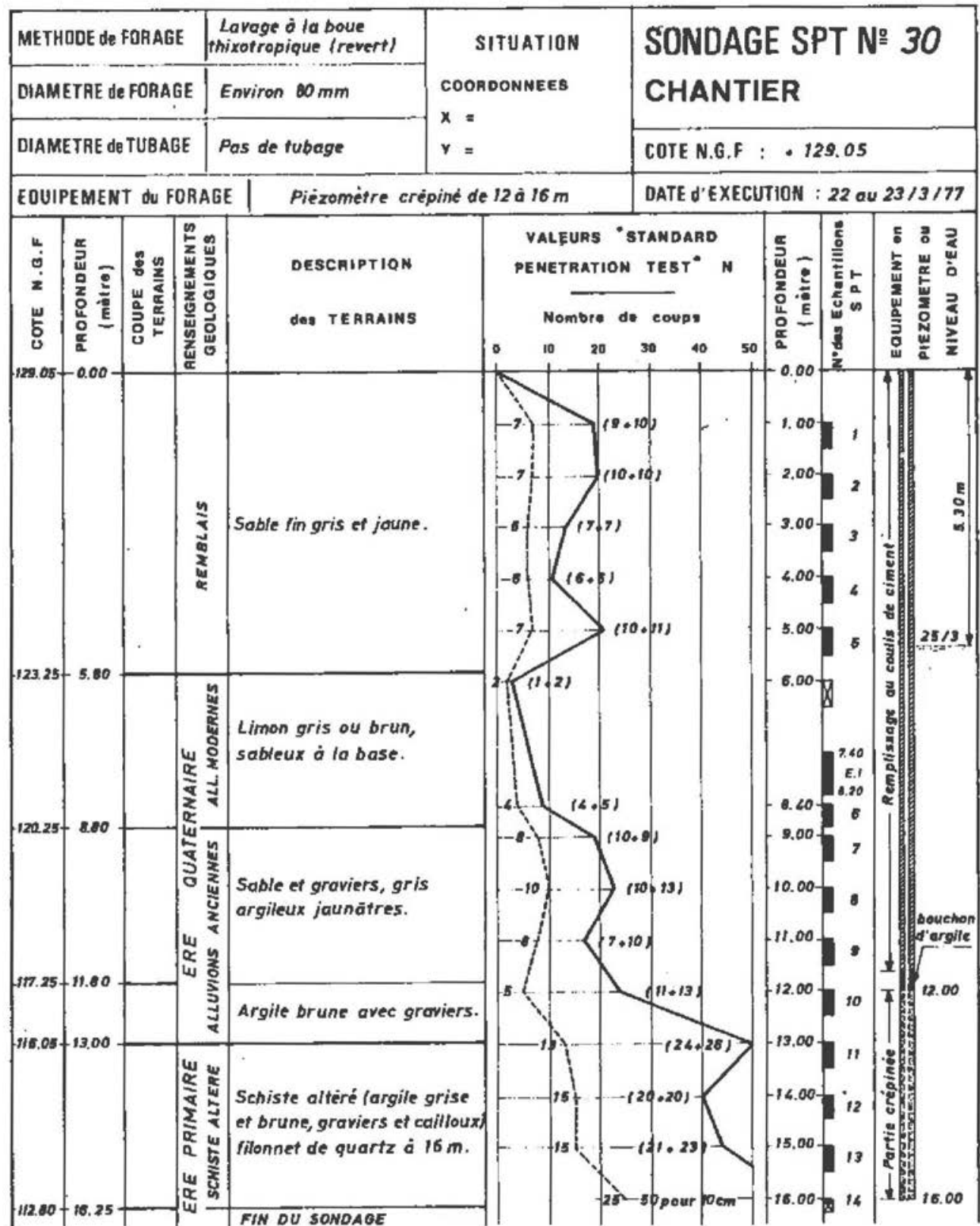

Ech. SPT recueilli (carottier plein\}

Ech, SPT non recueilli (carottier vide)

(E) Ech, SPT recueilli partiellement (caroltier non plein)

$\square_{2,20}^{2,40} \varepsilon_{, t}$ Ech. intact
Nombre de coups de 0 à $35 \mathrm{~cm}$

Nombre de coups de 15 à $45 \mathrm{~cm}$

(valeur SPT)

Les nombres de coups entre 15 et $30 \mathrm{~cm}$ ef 30 et $45 \mathrm{~cm}$ sont indiqués entre parenthèses 


\section{B. sondage au pénétromètre statique}

Peuvent également être mesurés :

-la force totale d'enfoncement et d'arrachage,

- le frottement latéral total,

- la force de frottement sur un manchon,

-la pression interstitielle au voisinage du cône,

- les déviations du train de tiges dans le sol.

Le penétrometre statique normalise, les pénétrometres traditionnels et le mode operatoire sont décrits par la norme européenne en vigueur, reproduite en annexe.

\section{Définitions}

1.1 Exécuter un sondage au pénétromètre statique consiste à faire pénétrer dans le sol, par vérinage à vitesse constante, une pointe conique portée par un train de tiges et à mesurer, de manière continue ou à intervalles déterminés, la résistance à la pénétration du cône.

1.2 La résistance à la pénétration du cône $q_{c}$ est obtenue en divisant la force sur le cône $Q_{c}$ lou effort de pointe) par la surface $A_{c}$ de la plus grande section droite de la pointe :

$a_{c}=\frac{Q_{c}}{A_{c}}$

\section{Caractéristiques et limites de l'appareillage}

2.1 La norme européenne recommande un pénétromètre-type. Si on utilise un autre appareil, on doit mentionner sur tes feuilles d'essai les caractéristiques qui le différencient du pénétromètre-type. 
Beaucoup d'appareils sont portés par un camion lesté dont le poids sert de réaction au vérin de fonçage.

L'attention est attirée sur les conditions d'accès et de mise en station du matériel aux emplacements prévus.

Lorsque la résistance totale du sol devient supérieure à la capacité de réaction du lest du verin de fonçage, le pénétromètre ne s'enfonce plus et I'essai statique est arrêté.

Dans certains cas, l'essai statique peut être repris dans le même sondage après pénétration par une autre méthode.

\section{On rappelle que :}

- la vitesse de fonçage ou vitesse de pénétration est fixée à $2 \mathrm{~cm} / \mathrm{s}$ $\pm 0,5 \mathrm{~cm} / \mathrm{s}$,

- I'intervalle entre deux lectures ne doit pas excéder $0,20 \mathrm{~m}$ de pénétration,

- les appareils de mesure doivent avoir les précisions définies par la normie européenne.

Un exemple de graphique est donné ci-après dans un cas où $q_{c}$ est supérieur à $2 \mathrm{MPa}$.

Cette indication ne prejuge pas du niveau statique de la nappe.
2.2 Le diamètre du cône, la force totale maximale et la force maximale sur le cône suisceptibles d'être développées par l'appareil, doivent figurer obligatoirement sur la proposition de l'entrepreneur et sur les feuilles d'essai.

2.3 Chaque sondage est conduit jusqu'à la profondeur indiquée par les documents particuliers du marché, exception faite des cas où la résistance du sol ạtteint les valeurs maximales définies en 2.2 ci-dessus.

Si un sondage ne peut atteindre la profondeur prévue, l'entrepreneur en avise le maître d'ouvrage, lequel prend les décisions nécessaires après consultation du géotechnicien.

\section{Procédure d'essai}

3.1 La procédure d'essai est fixée dans le mode opératoire de l'essai normalisé de la norme européenne.

3.2 Lorsqu'un appareil mécanique (à transmission par tiges) est utilisé, la valeur de la résistance de pointe doit être corrigée en tenant compte du poids des tiges.

\section{Présentation des résultats}

4.1 Les résultats d'un sondage au pénétromètre statiquée sont présentés sous forme d'un graphique donnant, exclusivement en échelle arithmétique, en fonction de la profondeur atteinte par le cône de pointe, la résistance à la pénétration du cône $q_{c}$ ainsi que les valeurs des autres paramétres qui peuvent avoir également été mesturés.

Le choix de l'échelle pour la représentation de $q_{C}$ doit permettre d'apprécier à $10^{5} \mathrm{~Pa}$ près les valeurs inférieures à $2 \mathrm{MPa}$.

\subsection{Le graphique porte les indications suivantes :}

- le type de l'appareil et de son système de mesure,

- le numéro d'identification du sondage,

- la ou les dates d'exécution,

- la cote de départ et, éventueliement, les coordonnées dans un système de référence précisé,

- l'indication des profondeurs et durées des éventuel-

les interruptions,

- le niveau de l'eau dans le sondage après extraction. 


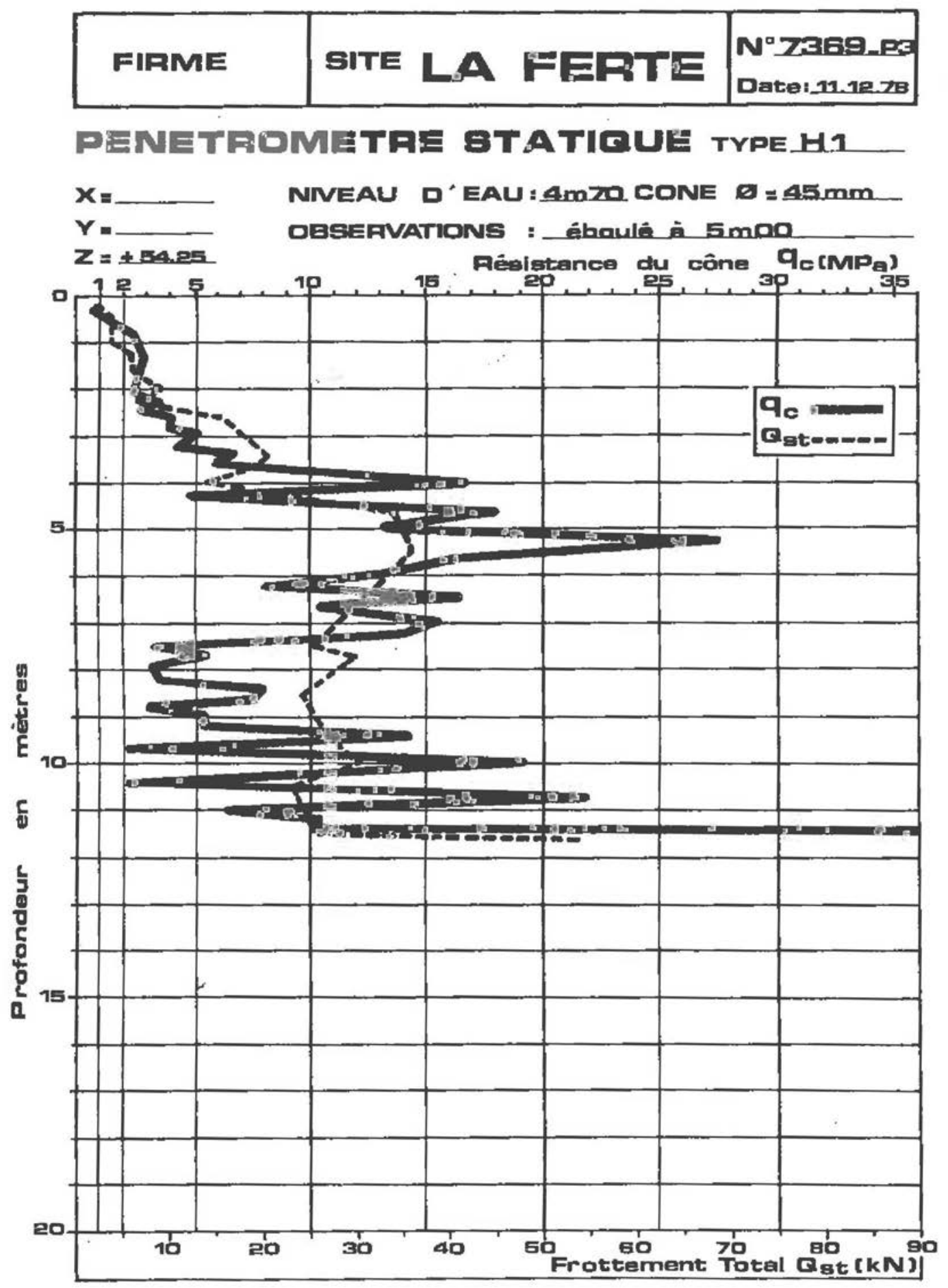




\section{C. sondage au pénétromètre dynamique}

Avec le type A, la procédure de l'essai supprime le frottement latéral du sol sur les tiges.

Toutefois, dans les argiles gonflantes ou dans les alluvions grossieres, le frottement latéral n'est pas toujours complètement éliminé par l'injection de bentonite surtout à grande profondeur.

Par ailleurs, avec les pénétromètres à tube de protection, des frottements parasites peuvent se manifester au voisinage de la pointe entre le tube de protection et les tiges intérieures, surtout dans les sols fins sous la nappe.

L'utilisation du nombre de coups " $N_{d a}$ défini dans la norme européen-

\section{Définitions}

1.1 Exécuter un sondage au pénétromètre dynamique consiste à faíre pénétrer dans le sol par battage une pointe métallique portée par un train de tiges et à mesurer à intervalles d'enfoncement réguliers l'énergie nécessaire correspondante.

1.2 Il existe un pénétromètre et un essai de référence dits de type $\mathrm{A}$ et décrits par la norme européenne en vigueur. Celle-ci définit également un essai simplifié dit de type $B$.

1.3 Tout pénétromètre dynamique comprend un mouton de masse $M$ tombant d'une hauteur fixe $H$. Le mouton frappe une enclume solidaire du train de tiges et produit ainsi l'enfoncement de la pointe. On note le nombre de coups " $\mathrm{N}_{\mathrm{d}}$ " nécessaire pour faire pénétrer la pointe sur une hauteur "h».

La résistance dynamique apparente $q_{d}$ est calculée par la formule des Hollandais qui s'écrit : 
ne n'est admise que dans le cas de l'utilisation d'un pénétromètre de type $A$. La résistance dynamique du sol $q_{d}$ doit être considérée comme purement conventionnelle.

On peut également utiliser la 2ème formule présentée dans la norme européenne à condition de désigner

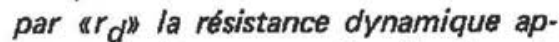
parente obtenue par l'application de ladite formule.
Dans le cas des pénétromètres à tube de protection, cette résistance dynamique apparente "limite» au-delà de laquelle le sondage ne peut généralement plus être poursuivi peut être celle correspondant au battage de l'ensemble tiges-tube.

Dans le cas où l'appareil et l'essai sont strictement conformes au type $A$ ou $B$, il suffit de noter sur la feuille d'essai : "essai DPA» ou "essai DPB».
$q_{d}=\frac{M}{M+M^{\prime}} \times \frac{M \cdot g \cdot H}{A \cdot \theta}$

dans laquelle :

$M^{\prime}$ est la masse frappée (somme des masses des tiges, de l'enclume, de la.tige guide et de la pointe),

A est la surface de la plus grande section droite de la pointe,

g est l'accélération de la pesanteur,

e est l'intervalie moyen d'enfoncement égal à $\frac{h}{N_{d}}$

\section{Caractéristiques et limites de l'appareillage}

2.1 Quel que soit le pénétromètre utilisé, le diamètre du cône, celui des tiges, la masse du mouton le plus lourd et la hauteur de chute maximale doivent figurer obligatoirement sur la proposition de l'entrepreneur.

2.2 Chaque sondage au pénétromètre dynamique est conduit jusqu'à la profondeur indiquée par les documents particuliers du marché, exception faite des cas où la résistance dynamique apparente $\mathrm{du}$ sol " $\mathrm{q}_{\mathrm{d}}$ " dépasse $100 \mathrm{MPa}$.

Si un sondage ne peut atteindre la profondeur prévue, I'entrepreneur en avise le maître d'ouvrage, lequel prend les décisions nécessaires après consultation du géotechnicien.

2.3 On doit mentionner sur les feuilles d'essai, les caractéristiques essentielles de I'appareil et du processus d'essai, en particulier les valeurs de tous les paramètres entrant dans le calcul de $\mathrm{q}_{\mathrm{d}}$.

\subsection{Mouton}

Le mouton est levé par un dispositif qui le libère automatiquement en position haute de façon que sa hauteur de chute soit constante et sa vitesse initiale négligeable. La chute du mouton est absolument libre.

\subsection{Enclume ot tige-guide}

L'enclume et la tige-guide sont parfaitement solidaires entre elles et avec le train de tiges. L'axe de l'enclume, de la tige-guide et du train de tiges est unique et vertical. 
II est conseillé que les raccords aient une section d'acier la plus voisine possible de celle du train de tiges correspondant.
L'annulation du frottement latéral étant aléatoire dans le cas de la procédure du type $B$, le sondage de type $B$ ne doit étre utilisé que comme un moyen de reconnaissance sommaire.

La présentation d'un graphique du nombre de coups $N_{d a}$ pour l'essai de type $A$ de la norme européenne, ne dispense pas de la présentation d'un graphique de $q_{d}$.

Une représentation logarithmique peut être utilisée en complément si les variations de résistance dynamique sont très grandes d'un niveau de sol au suivant.

On indiquera également, si c'est le cas, l'absence d'eau et/ou le niveau auquel le sondage est obstrué après extraction des tiges.

Cette indication ne préjuge pas du niveau statique de la nappe.

\subsection{Tiges}

Les tiges sont lisses, bien droites et leur assemblage se fait de manière à éviter tout jeu au niveau des raccords. Les raccords ont le même diamètre extérieur que les tiges. Celles-ci sont marquées, soit de manière permanente, soit avant leur battage, à intervalles réguliers correspondant à «h».

\subsection{Pointe}

La pointe a une forme cylindro-conique. Le cylindre a un diamètre égal à sa hauteur. Le cône a un angle au sommet de $90^{\circ}$. Le diamètre de la pointe est supérieur à celui du train de tiges de $20 \mathrm{~mm} \pm 2 \mathrm{~mm}$.

\subsection{Procédures d'essai}

Les procédures d'essai sont fixées par la norme européenne.

\section{Présentation des résultats}

3.1 Les résultats d'un sondage au pénétromètre dynamique sont présentés sous forme d'un graphique donnant en échelle arithmétique les variations de la résistance dynamique apparente $q_{d}$ en fonction de la profondeur atteinte par la pointe.

Le choix de l'échelle pour la représentation de $q_{c}$ doit permettre d'apprécier à $10^{5} \mathrm{~Pa}$ près les valeurs inférieures à $2 \mathrm{MPa}$.

\subsection{Le graphique porte les indications suivantes:}

- le type de l'appareil et de son système de mesure,

- le numéro d'identification du sondage,

- la ou les dates d'exécution,

- la cote de départ et, éventueilement, les coordonnées dans un système de référence précisé,

- l'indication des profondeurs et durées des éventuelles interruptions,

- le niveau de l'eau dans le sondage après extraction. 


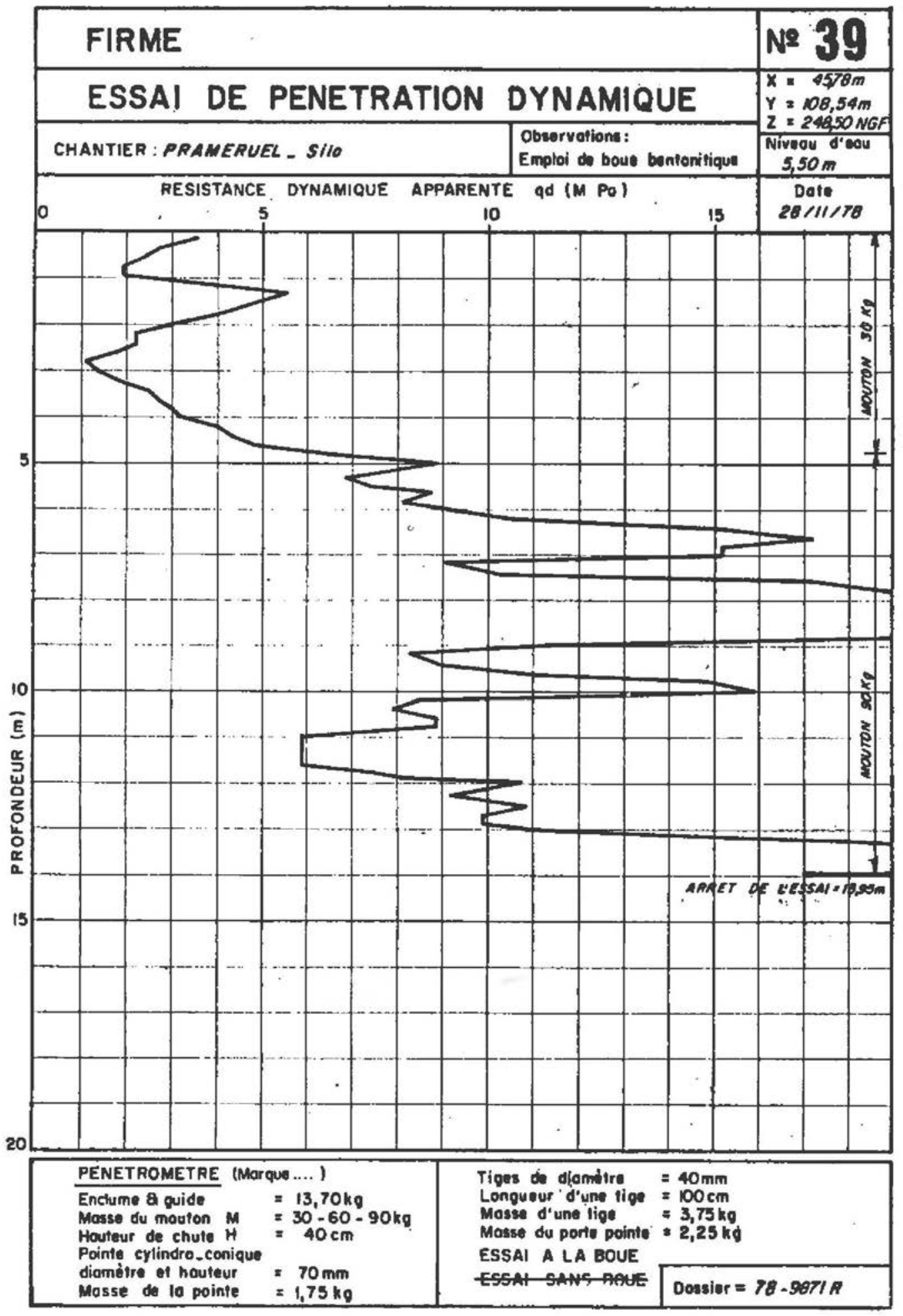




\section{D. sondage pressiométrique normal}

\section{Définitions}

\subsection{Sondage pressiométrique normal}

On appelle sondage pressiométrique l'ensemble des deux opérations suivantes :

- le forage permettant la mise en place correcte de la sonde pressiométrique normale,

- la réalisation des essais pressiométriques propre. ment dits.

\subsection{Essai pressiométrique normal}

L'essai pressiométrique normal est un essai de chargement statique du sol en place, réalisé à l'aide d'une sonde cylindrique dilatable radialement (voir article 3.1). II permet d'obtenir une relation contrain. te-déformation du sol en place dans l'hypothèse d'une déformation plane et de déterminer les trois para. mètres usuels suivants :

Certaines procédures d'essai permet- un module de déformation du sol, tent la détermination de modules particuliers (alterné, retour, etc.).

- une pression dite "pression de fluage»,

- une pression dite «pression limite».

\section{Mise en place des sondes}

\subsection{Prescriptions générales}

2.1.1 La méthode et le matériel utilisés doivent ètre choisis de telle sorte que :

- le sol à la paroi demeure pratiquement intact,

- le diamètre du forage soit adapté au diamètre de la sonde, 
Un tableau récapitulatif donné en annexe précise les différentes méthodes de forage à amployer selon la nature des sols.

Les valeurs maximales tolérées des parametres de forage sont les suivantes:

- couple : 200 m.N

- vitesse de rotation : $60 \mathrm{tr} / \mathrm{mn}$

- pression sur l'outil : 0,2 MPa

-pression d'injection de boue :

$0,5 \mathrm{MPa}$

- débit d'injection : 15 litres/mn
Dans le cas de sondages à la tariere, il faut veiller à ce que loutil soit parfaitement affutté et à ce que le sol soit découpé et non refoulé.
- la réalisation du forage soit conforme aux prescrip tions de l'article suivant 2.2.

2.1.2 Les essais doivent être réalisés immédiatemen après l'exécution d'une longueur de forage. La lon gueur maximale de forage autorisée avant réalisatior des essais correspondants est limitée dans les para graphes suivants en fonction de la nature des terrains traversés.

\subsection{Réalisation du forage}

Les méthodes de réalisation du forage dépendent de la nature des sols étudiés :

\subsubsection{Vases et argiles molles}

2.2.1.1 Le forage est réalisé soit à la tarière à main avec injection axiale simultanée de boue sous faible pression, soit éventuellement avec un outil rotatif désagrégateur dans des conditions analogues à celles de la tarière à main.

L'injection de boue est maintenue pendant la remontée de l'outil.

2.2.1.2 Tout forage avec injection d'eau claire est interdit.

2.2.1.3 La mise en place de la sonde par refoulement du sol est interdite.

2.2.1.4 La longueur maximale de forage autorisée ne sera pas supérieure à l'intervalle entre essais.

En cas de déformation de la paroi du forage empêchant l'introduction libre de la sonde, le tubage préalable de la partie du forage située au-dessus de la zone d'essai est indispensable.

\subsubsection{Argiles moyennement compactes}

2.2.2.1 Le forage est réalisé à la tarière à main à sec ou avec injection de boue, à la tarière hélicoïdale continue à outil d'attaque légèrement débordant ou à l'outil désagrégateur avec injection de boue.

2.2.2.2 Tout forage avec injection d'eau claire est interdit.

2.2.2.3 La mise en place de la sonde par refoulement du sol est interdite.

2.2.2.4 La longueur maximale de forage autorisée avant réalisation des essais correspondants est de $5 \mathrm{~m}$. 
Le soufflage axial d'air comprimé est inopérant au-delà d'une certaine teneur en eau du sol.

On veille en particulier à éviter toute interruption de la circulation du fluide de forage.

Les valeurs maximales tolérées des parametres de forage sont les suivantes:

- couple : 200 m.N

- vitesse de rotation : $60 \mathrm{tr} / \mathrm{mn}$

- pression sur l'outil :0,2 MPa

-pression d'injection de boue :

$0,5 \mathrm{MPa}$

- débit d'injection : 15 litres/mn

Les valeurs maximales tolérées des parametres de forage sont les suivantes:

- couple : 200 m.N

- vitesse de rotation : $60 \mathrm{tr} / \mathrm{mn}$

- pression sur l'autil : 0,2 MPa

- pression d'injection de boue :

$0,5 \mathrm{MPa}$

- débit d'injection : 15 litres/mn

On veille en particulier à éviter toute interruption de la circulation du fluide de forage.

\subsubsection{Argiles compactes - marnes raides}

2.2.3.1 Le forage est réalisé soit à l'outil désagrégateur rotatif ou éventueliement rotopercutant avec soufflage axial d'air comprimé ou injection de boue, soit à la tarière hélicoïdale continue avec outil d'attaque légèrement débordant, soit par earottage avec injection de boue.

2.2.3.2 Tout forage avec injection d'eau claire est interdit.

2.2.3.3 La mise en place de la sonde par refoulement du sol est interdite.

2.2.3.4 Tout forage par refoulement du soi est interdit.

2.2.3.5 La longueur maximale de forage autorisée est celle qui permet la réalisation de tous les essais pressiométriques correspondants durant le même poste de travail.

\subsubsection{Limons}

\subsubsection{Limons au-dessus de la nappe}

Le forage est réalisé soit à la tarière à main, à sec ou éventuellement avec injection de boue, soit à la tarière hélicoïdale continue ou, éventuellement, à l'outil désagrégateur avec injection de boue.

Le battage ou le vibrofonçage d'un carottier est toléré.

\subsubsection{Limons sous la nappe}

Le forage est réalisé à la tarière à main avec injection de boue ou éventuellement soit à l'outil désagrégateur avec injection, soit par carottage avec injection de boue.

2.2.4.3 Tout forage avec injection d'eau claire est interdit.

2.2.4.4 La mise en place de la sonde par refoulement du sol est interdite.

2.2.4.5 La longueur maximale de forage autorisée est ceile qui permet la réalisation de tous les essais pressiométriques correspondants durant le même poste de travail. 
En cas d'utilisation de la tarière helicoidale continue, la vitesse de pénétration, lente, est choisie de manière à ne provoquer aucun refoulement de terrain.

Les valeurs maximales tolérées des paramètres de forage à l'outil rotatif désagrégateur sont les suivantes :

- couple : $200 \mathrm{~m} . \mathrm{N}$

- vitesse de rotation : $60 \mathrm{tr} / \mathrm{mn}$

-pression d'injection de boue : $0,5 \mathrm{MPa}$

- débit d'injection : 15 litres/mn

- pression sur l'outil : 0,2 MPa

Lorsque ces matériaux peu compacts contiennent de gros éléments, il est nécessaire de battre le pressiomètre sans avant-trou, l'essai se faisant à I'interieur d'un tube fendu.

Les valeurs maximales tolérées des parametres de forage à l'outil rotatif désagrégateur sont les suivantes :

- couple : 200 m.N

- vitesse de rotation : $60 \mathrm{tr} / \mathrm{mn}$

- pression d'injection de boue

$0,5 \mathrm{MPa}$

- debit d'injection : 15 litres/mn

- pression sur l'outil : 0,2 MPa

Les valeurs maximales tolérées des paramètres de forage à l'outil rotatif désagrégateur sont les suivantes :

- couple : 200 m.N

- vitesse de rotation : $60 \mathrm{tr} / \mathrm{mn}$

-pression d'injection de boue :

$0,5 \mathrm{MPa}$

- débit d'injection : 15 litres/mn

- pression sur l'outil : 0,2 MPa

\subsubsection{Sables láches}

\subsubsection{Situés au-dessus de la nappe}

Le forage est réalisé à la tarière à main avec ou sans injection de boue ou éventuellement soit avec une tarière hélicoïdale continue à sec en maintenant la rotation aussi bien à la descente qu'à la remontée, soit à l'outil rotatif désagrégateur avec injection de boue dans des conditions analogues à celles de la tarière à main.

\subsubsection{Situés sous la nappe}

Le forage est réalisé à la tarière à main avec injection axiale simultanée de boue sous faible pression, ou, éventuellement, avec un outil rotatif désagrégateur dans des conditions analogues à celles de la tarière à main.

2.2.5.3 Tout forage avec injection d'eau claire est interdit.

2.2.5.4 La longueur maximale de forage autorisée avant réalisation des essais correspondants est de $5 \mathrm{~m}$.

\subsubsection{Sables moyennement compacts et compacts}

2.2.6.1 Le forage est réalisé soit à la tarière à main, avec ou sans injection de boue, soit à la tarière hélicoïdale continue, soit à l'outil désagrégateur avec injection axiale de boue.

Dans les sables compacts, on peut utiliser la rotopercussion soit avec soufflage axial d'air comprimé, soit avec injection de boue lorsque les sables sont sous la nappe.

Le battage ou le vibrofonçage d'un carottier sont tolérés. 
Le soufflage axial d'air comprimé est inopérant au-delà d'une certaine teneur en eau du sol.

Lorsque ces matériaux, sous la nappe, contiennent de gros éléments, on peut réaliser l'essai dans un tube fendu mis en place par battage ou par vibrofoncage.

Lorsque la mise en place du tube fen. du ne peut plus être faite par battage ou par vibrofonçage, on réalise au préalable un forage pilote de plus petit diametre, puis on reprend la mise en place normale du tube fendu.

Lorsque la mise en place du tube fendu ne peut plus être faite par battage ou par vibrofonçage, on réalise au préalable un forage pilote de plus petit diametre, puis on reprend la mise en place normale du tube fendu.

Dans les roches fracturées, la sonde peut être descendue à l'abri d'un tube fendu de protection.
2.2.6.2 La longueur maximale de forage autorisée est celle qui permet la réalisation de tous les essais pressiométriques correspondants durant le même poste de travail.

\subsubsection{Sols grossiers}

2.2.7.1 Entrent dans cette catégorie :

- les sables et graviers,

- les galets, cailioux et blocs,

- les remblais grossiers.

2.2.7.2 Le forage est réalisé par l'une des méthodes suivantes :

- tarière hélicoïdale continue,

- outil désagrégateur rotatif avec injection centrale de boue,

- outil désagrégateur rotopercutant avec soufflage axial d'air comprimé,

- battage ou vibrofonçage d'ún carottier.

Lorsque l'équilibre des parois n'est pas assuré, il faut recourir à l'emploi du tube fendu. Sa mise en place est faite par battage ou par vibrofonçage.

2.2.7.3 La longueur maximale de forage autorisée est celle qui permet la réalisation de tous les essais pressiométriques correspondants durant le même poste de travail.

\subsubsection{Roches}

2.2.8.1 Le forage est réalisé par toute méthode habituelle permettant d'obtenir un trou bien calibré.

2.2.8.2 La longueur maximale de forage autorisée est celle qui permet la réalisation de tous les essais pressiométriques correspondants durant le même poste de travail. 
On peut se référer au mode opératoire MS.IS.2 publié par le Laboratoire Central des Ponts et Chaussées sous le titre "Essai Pressiométrique Norma/».

\section{Réalisation de l'essai pressiométrique normal}

\subsection{Sonde pressiométrique normale}

La sonde pressiométrique normale est une sonde constituée de trois parties agissant simultanément durant I'essai sur la paroi du forage :

- une cellute centrale de mesure,

- deux cellules de garde, de même diamètre que la précédente, situées de part et d'autre de la cellule centrale, destinées à assurer un champ de déformation cylindrique sur la hauteur de la cellule centrale.

La hauteur de la cellule de mesure doit être au moins égale à 3 (trois) fois le diamètre nominal de la sonde et inférieure à $50 \mathrm{~cm}$.

La hauteur de chaque cellule de garde doit être au moins égale à 1,5 (un virgule cinq) fois le diamètre nominal de la sonde.

\subsection{Déroulement de l'essai}

3.2.1 Une fois la sonde mise en place, on lui applique des paliers de pression croissants, chaque palier étant maintenu une minute, et on note les déforma: tions volumétriques correspondantes, à 30 secondes et une minute au cours de ce palier.

3.2.2 Le nombre de paliers doit être au moins égal à 7 (sept).

3.2.3 Pour chaque essai, on trace la courbe pressiométrique qui traduit la variation de la déformation volumétrique de la cellule de mesure en fonction de la pression appliquée.

3.2.4 Pour s'assurer de la validité de l'essai, on vérifie l'ordre de grandeur de la déformation volumétrique de la sonde correspondant au contact avec la paroi du forage. Cette déformation volumétrique doit être inférieure à la moitié du volume initial de la sonde $\left\{\frac{\Delta V}{V_{0}} \leqslant 0,5\right\}$

3.2.5 Les corrections à réaliser sont les suivantes :

3.2.5.1 Correction de pression pour un volume donné, afin de tenir compte de la pression propre de déformation de l'ensemble membrane-gaine plus éventuellement tube fendu (courbe d'étalonnage).

3.2.5.2 Correction de volume pour une pression donnée, destinée à éliminer les déformations internes des différents organes constituant l'appareil. 
Aucune correction n'est nécessaire à I'́gard du niveau statique de la ou des nappes d'eau, les mesures étant exprimées en contraintes totales.

Un espacement de $1,00 \mathrm{~m}$ entre essai représente l'espacement souhaitable.

On peut, sur ce point, se reférer au chapitre "Calculs et resultats" du mode opératoire MS.IS.2 publié par le Laboratoire Central des Ponts et Chausaées sous le titre «Essai Pressiométrique Normaly.

Un exemple de coupe de sondage pressiométrique est donné en annexe.

Les coordonnées du point de référence fixe doivent être rapportées au système gédésique local officiellement en vigueur.
3.2.5.3 Les pressions lues doivent être corrigées de la différence de charge hydrostatique correspondant à la position relative du manomètre et de la cellule de mesure.

\section{Fréquence des essais}

La fréquence des essais, c'est-à-dire le nombre d'essais pressiométriques par sondage, est fixée par le maître d'ouvrage ou ses représentants sur proposition du géotechṇicien.

Les essais ne doivent pas être espacés de plus de 1,5 m au cours d'un même sondage.

\section{Dépouillement des essais}

Le dépouillement des essais et le calcul des caractéristiques pressiométriques définies à l'article 1 du présent sous-chapitre. sont faits par l'entreprise qui a réalisé les essais.

\section{Présentation des résultats}

Les résultats des essais, remis au maître d'ouvrage ou à ses représentants, sont présentés sous forme d'une coupe de sondage pressiométrique comportant les éléments suivants :

- la désignation du chantier,

- le nom et l'adresse de l'entreprise de sondage,

- la numérotation du sondage et la référence du plan de situation permettant de le repérer,

- la date du début et de la fin de l'exécution du sondage,

- le repérage en profondeur et en altitude par rapport à un point de référence fixe, des renseignements portés sur la coupe de sondage,

- la description sommaire des terrains traversés,

- la ou les méthodes de perforation avec leur profondeur d'utilisation et la nature de l'outil utilisé,

- le ou les diamètres du trou,

- l'indication du fluide de perforation,

- la résistance à l'avancement (nombre de coups pour 'un enfoncement donné) en cas d'utilisation d'un dispositif de forage par battage,

- les observations concernant les arrivées d'eau

et les pertes du fluide de perforation,

- l'indication des niveaux des essais pressiométriques

effectués,

- le ou les types de sonde utilisée, 
Cette indication ne prejuge pas du niveau statique de la nappe.
- les valeurs des caractéristiques pressịométriques calculées à chacun de ces niveaux,

- le niveau de l'eau ou de la boue dans le sondage en fin d'exécution,

- les renseignements éventuels sur l'équipement piézométrique (voir article 7 du chapitre II du présent cahier des charges).

\section{Carnet de mesures}

Si le mâtre d'ouvrage ou ses représentants l'exigent, I'entrepreneur est tenu de fournir les carnets de mesures sur lesquels figurent en particulier les mesures relatives à l'étalonnage des sondes.

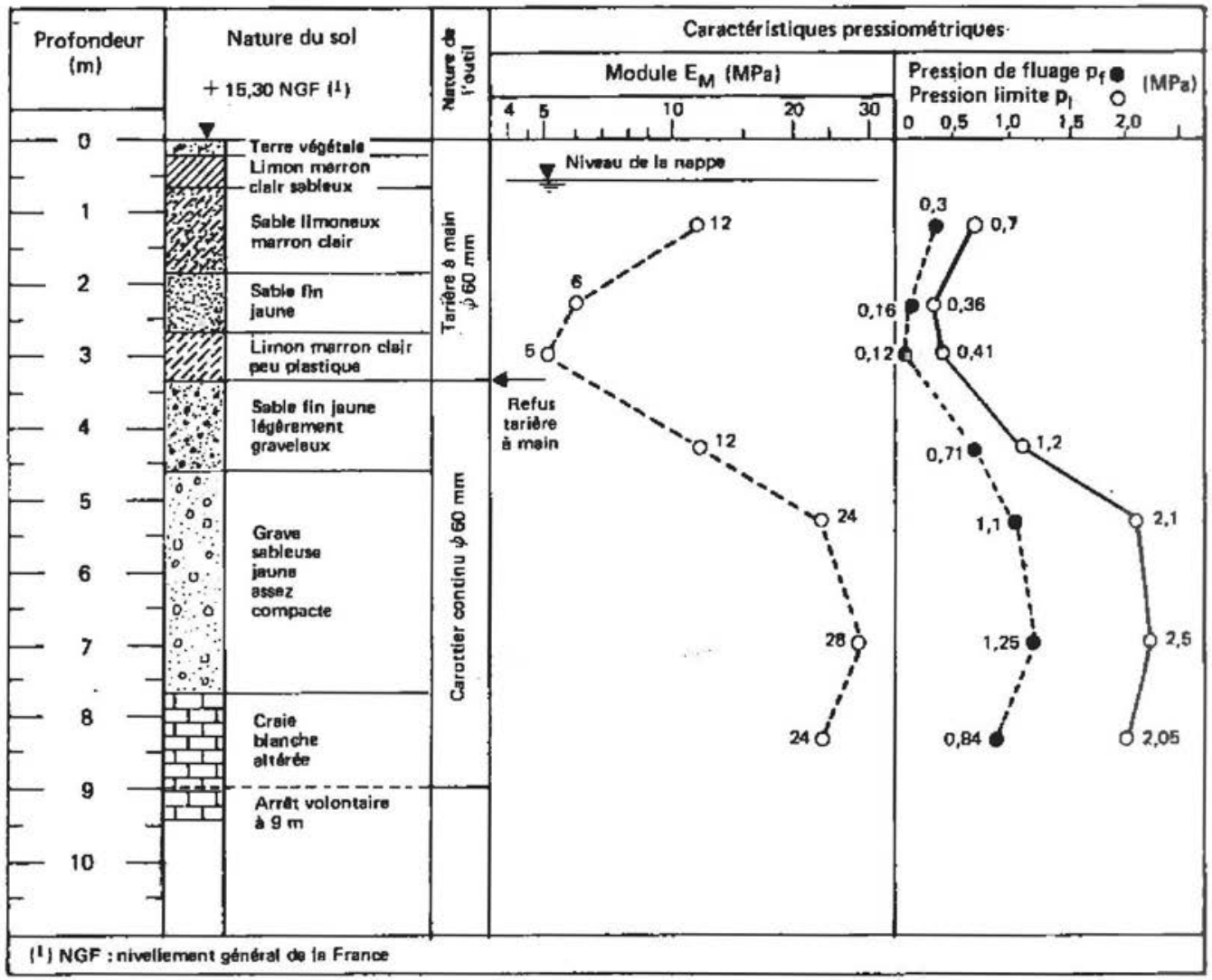




\begin{tabular}{|c|c|c|c|c|c|c|c|c|c|}
\hline \multirow{2}{*}{$\begin{array}{l}\text { MATURR DES } \\
\text { TRRRANINS }\end{array}$} & \multicolumn{5}{|c|}{ HOTATIOM } & \multicolumn{4}{|c|}{ MATTAS:P, RT AITTRKS } \\
\hline & TNM.S & TMA.IN & $\mathrm{mic}$ & $0 . \mathrm{NG}, \mathrm{IN}$ & CAN. & W.D. ROTOP & CNA. ANT & CAR. VUP & Tr ant/vir \\
\hline VNSR WT NRGTLES MOLINS & $=$ & & $=$ & & & & & & \\
\hline $\begin{array}{l}\text { ARGLLES MOYRNNFORSTIT } \\
\text { COMPNCTRS }\end{array}$ & & & & & & 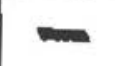 & & & \\
\hline $\begin{array}{l}\text { ARGTLSS COMPACTES } \\
\text { MARARS RAIDRS }\end{array}$ & & & & & & & & & \\
\hline $\begin{array}{l}\text { LIMOHS } \\
- \text { - AV-DESSOB DE SA MAPPR }\end{array}$ & & & & & & & & & \\
\hline - SOUS LA MAPPZ & & & & & & & & $\rightarrow$ & \\
\hline $\begin{array}{l}\text { BABLLES LACHES } \\
\text { - AU-DRESUS DE LA MAPPE }\end{array}$ & & & & & & 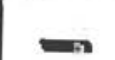 & & & \\
\hline - SOUS LA RAPPR & & & con & & & & & & \\
\hline $\begin{array}{l}\text { SALLRS MOVENHERENT } \\
\text { COMPACTS ET COMPACTS }\end{array}$ & & & & & & 0 & & & \\
\hline $\begin{array}{l}\text { 80LS GROBSIKRS - } \\
\text { GRAVIRRS, GALRTS, ETC. }\end{array}$ & & & & & & & & & \\
\hline $\begin{array}{l}\text { ROCHES } \\
\text { - MLTERRES }\end{array}$ & & & & & & & & & \\
\hline - satmps & & & & & & , & & & \\
\hline
\end{tabular}

- dann certaines conditions (voir texte)

\section{ABREVIATIONS}

I recommande

() TOlere

- INTERDIT

/inadapte

o

VOIR TEXTE
TAM.S TAReiplek A MAIN

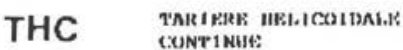

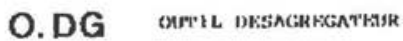

W.D.ROTOP. WAcion ins Il.t.

BAT. marracis

TF TAM.IN TARIIEE: A MAIN

CAR. canorrtikie

IN. AVRC tN.HECrato

POINCุ P(Inçonnmun

VBF vinkorongcasik 
Courbe d'expansion

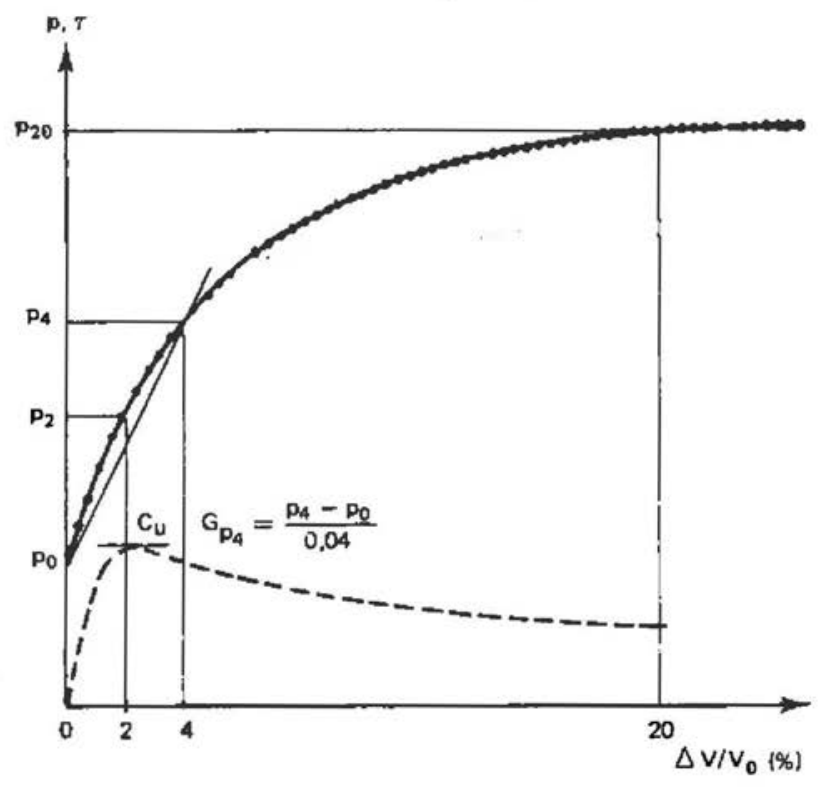




\section{E. sondage pressiométrique}

\section{au pressiomètre autoforeur}

$V_{0}$ représente le volume initial de la sonde,

$\Delta V$ représente la variation du volume de cette sonde soumise à une pression p.

Le pressiometre autoforeur permet d'étudier le comportement du sol dans des conditions de chargement variées : essais rapides ou lonts, essais cycliques, etc.

Un essai normalisé dit "essai de référencey a été défini et fait l'objet d'un mode operatoire publié par le Laboratoire Central des Ponts et Chaussées.

\section{Définitions}

1.1 L'essai pressiométrique au pressiomètre autoforeur

L'essai au pressiomètre autoforeur est un essai de chargement du sol en place. II consiste à dilater une sonde cylindrique dans le terrain, ce qui permet de déterminer une relation pression-déformation $\left(\mathrm{p}, \frac{\Delta \mathrm{V}}{\mathrm{V}_{0}}\right.$ ).

De cette relation, on déduit les paramètres suivants :

$-p_{0}$ : pression initiale

- $p_{5}$ et $p_{20}$ : pression correspondant à des déformations $\frac{\Delta V}{V_{0}}$ respectivement de 5 et $20 \%$,

- les modules de cisaillement $G_{p 2}$ et $G_{p 5}$ correspondant à des déformations $\frac{\Delta V}{V_{0}}$ respectivement
de 2 et $5 \%$,

- $\beta$ coefficient d'identification des sols défini par la relation: $\quad \beta=\frac{p_{20}-p_{5}}{p_{20}-p_{0}} \quad$ (Figure ci-contre)

1.2 Le sondage pressiométrique au pressiomètre autoforeur

On appelle sondage pressiométrique au pressiomètre autoforeur l'ensemble des deux opérations suivantes :

- la mise en place de la sonde par autoforage,

- l'essai d'expansion proprement dit. 
Les caractéristiques de la pompe d'injection du fluide de forage sont :

- débit : 60 litres/minute

- pression : de l'ordre de $3000 \mathrm{kPa}$.

L'autoforage est inopérant dans les sols grossiers et les roches saines.

La traversée des couches de sols comportant des graves ou des galets est faite sous protection d'un tubage.

La rotation de l'outil est obtenue par un moteur hydraulique incorporé au module lui-merme.

L'élancement de ce module est généralement voisin de 2.

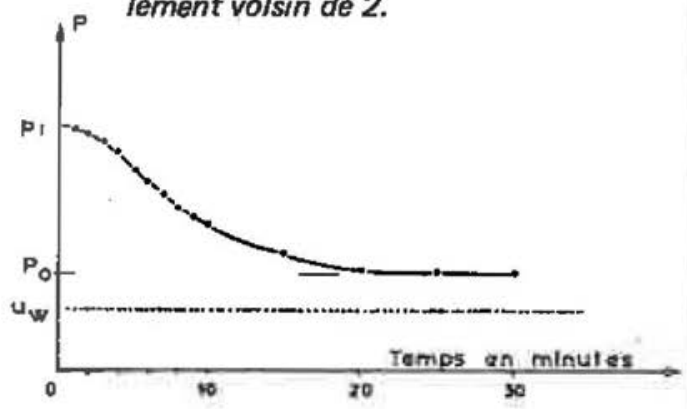

Cette stabilisation est généralement obtenue au bout de 10 minutes. Les pressions sont lues aux temps 0-1-2. 3-4-5 et 10 minutes puis toutes les 10 minutes au-delà.

Cette vitesse est contrôlese avec une précision de 1 \% sur l'étendue de l'échelle de la mesure.

\section{Mise en place de la sonde.}

La mise en place de la sonde se fait par autoforage, c'est-à-dire sans forage préalable, I'appareil étant équipé pour assurer par lui-même cette fonction d'introduction dans le sol.

Les sédiments produits par l'opération d'autoforage remontent à l'air libre à l'intérieur de la sonde à l'aide d'un fluide ou boue de forage.

\section{Réalisation de l'essai}

\subsection{Sonde du pressiomètre autoforeur}

La sonde du pressiomètre autoforeur comprend un empilement de 3 modules séparés :

- le module d'autoforage

C'est la partie inférieure de la sonde. If est constitué par un carottier à paroi mince à l'intérieur duquẹl se trouve l'outil désagrégateur permettant la destruction du sol.

- le module de mesure

II s'agit d'une cellule dilatable éventuellement protégée par un revêtement métailique.

- le module de poussée

C'est la partie supérieure de la sonde sur laquelle viennent se visser les tiges de forage.

\subsection{Déroulement de l'essai normalisé}

\subsubsection{Relaxation}

Une fois la cote d'essai atteinte, on arrête, dans l'ordre, le fonçage, la rotation, I'injection et on suit l'évolution de la pression de la cellule jusqu'à stabilisation.

\subsubsection{Essai d'expansion de référence}

Une fois l'opération de relaxation terminée, on procède à l'essai d'expansion. Celui-ci consiste à dilater la cellule à déformation contrôlée en lui appliquant une vitesse régulière de déformation $\frac{\Delta V}{V_{0}}=2 \%$ par minute jusqu'à une déformation maximale de $25 \%$. 
Cette vitesse correspond à un essai rapide de type non drainé dans les sols cohérents et drainé dans les sables propres.

Un espacement de 1,00 m entre essai represente l'espacement souhaitable.

On pout, sur ce point, se référer au chapitre "Calculs et resultatsy du mode operatoire publié par le Laboratoire Central des Ponts et Chaussées.

Un exemple de coupe de sondage au pressiometre autoforeur est donné en annexe.
3.2.3 Pour chaque essai, on trace la courbe de relaxation en fonction du temps et la courbe d'expansion.

3.2.4 On doit vérifier que les trois critères suivants sont respectés :

- la pression $p_{i}$ dans la sonde au moment de l'interruption du fonçage et la pression $p_{o}$ dans la sonde en fin de relaxation sont supérieures à la pression hydrostatique régnant dans le sol-à la profondeur de l'essai,

$-p_{i}-p_{0}$ est inférieure ou au plus égale à $\Delta p_{m}$. $\Delta \mathrm{p}_{\mathrm{m}}$ correspondant à la pression obtenue pour le premier incrément de volume,

- les coubes de relaxation et d'expansion doivent présenter une allure monotone, telle que celles représentées sur les figures précédentes sans point singulier.

3.2.5 Les courbes d'expansion doivent être corrigées pour tenir compte de la pression propre de déformation de la cellule de mesure.

Cette pression doit être déterminée par étalonnage.

\section{Fréquence des essais}

La fréquence des essais, c'est-à-dire le nombre d'expansions par sondage, est fixée par le maître d'ouvrage ou ses représentants sur proposition du géotechnicien.

Les essais ne doivent pas être espacés de plus de 1,5 m au cours d'un même sondage.

\section{Dépouillement des essais}

Le dépouillement des essais et le calcul des paramètres définis à l'article 1 du présent sous-chapitre sont faits par l'entreprise qui a réalisé les essais.

\section{Présentation des résultats}

Les résultats des essais remis au maître d'ouvrage ou à ses représentant's sont présentés sous forme d'une coupe de sondage au pressiomètre autoforeur comportant les éléments suivants : 
Les coordonnées du point de référence fixe doivent Gtre rapportées au systè. me géodésique local officiellement en vigueur.

Cette indication ne prejuge pas du niveau statique de la nappe.
- la désignation du chantier.

- le nom et l'adresse de l'entreprise de sondage,

- la numérotation du sondage et la référence du plan de situation permettant de le repérer,

- la date du début et de la fin de l'exécution du sondage,

- le repérage en profondeur et en al titude, par rapport à un point de référence fixe des renseignements portés sur la coupe de sondage,

- la description sommaire des terrains traversés,

- le type et le diamètre du pressiomètre autoforeur utilisé,

- I'indication du fluide de forage,

- le niveau où a été effectué chaque essai d'expansion,

- les valeurs des paramètres mesurés à chaque niveau testé,

- le niveau de l'eau ou de la boue dans le sondage après remontée de la sonde,

- les renseignements éventuels sur l'équipement piézométrique (voir article 7 du chapitre 11 du présent cahier des charges).

\section{Carnet de mesures}

Si le maître d'ouvrage ou ses représentants l'exigent, I'entrepreneur est tenu de fournir les carnets de mesures sur lesquels figurent en particulier les mesures relatives à l'étalonnage. 


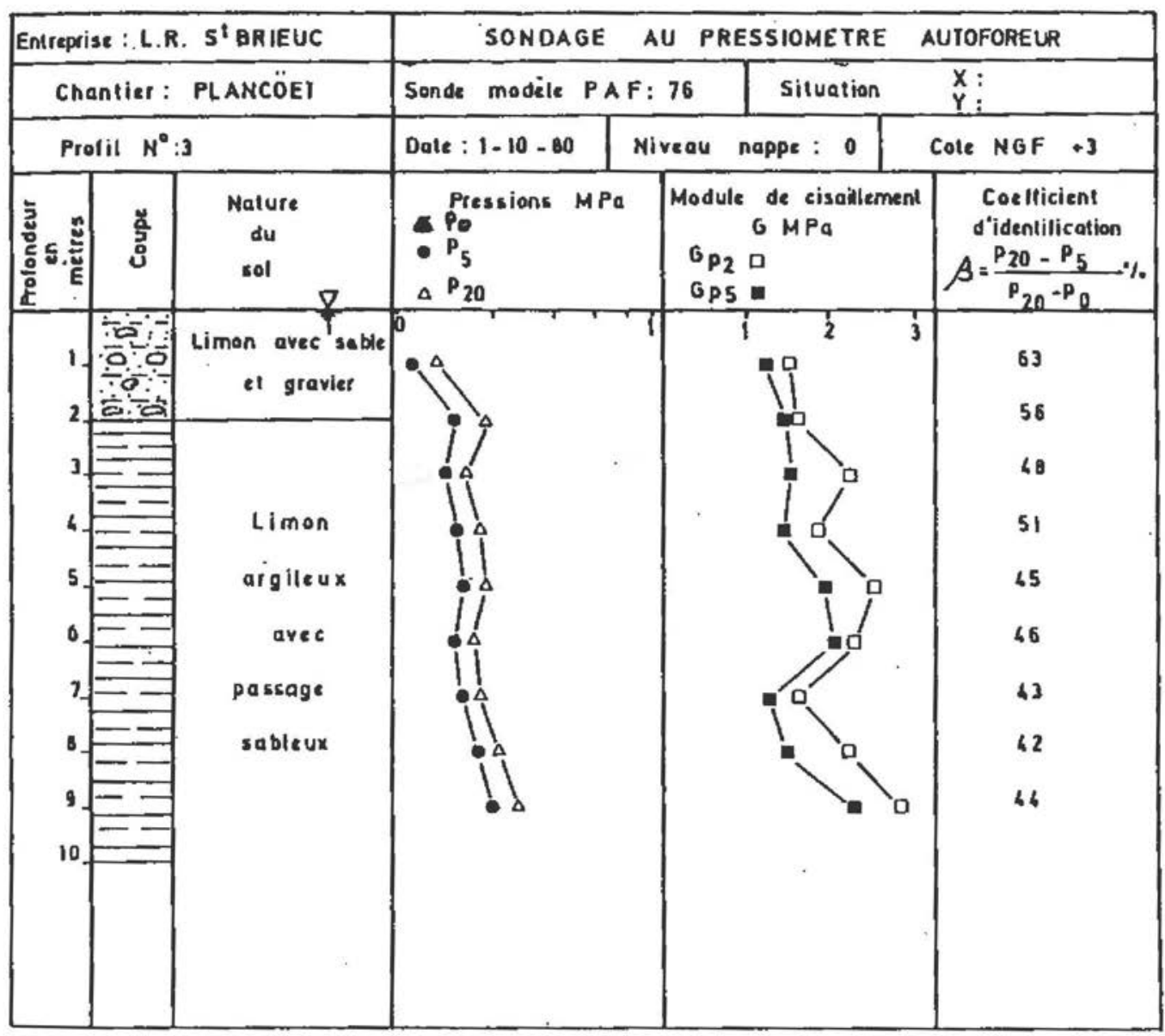




\section{F. l'essai au scissomètre}

L'essai au scissometre est limité aux sols fins cohérents dont la cohésion non drainée est $\leqslant 0,1 \mathrm{MPa}$.
Les principales hypothèses sont :

- l'essai est non drainé, :

- aucune perturbation n'est produite par le forage ou la mise' en place du scissometre,

- la zone remaniée autour des pales est très petite,

- II n'y a pas de rupture progressive,

- la résistance du sol est isotrope,

- la surface de rupture correspond à la géométrie des pales.

On peut se referer au mode opératoire du Laboratoire Central des Ponts et Chaussées.

\section{Définitions}

1.1 Exécuter un essai au scissomètre consiste à faire pénétrer dans un sol fin cohérent un moulinet à quatre pales orthogonales entre elles porté par un train de tiges puis à cisailler ce sol autour de ces pales par une mise en rotation de ce moulinet.

1.2 Cet essai permet de mesurer :

1.2.1 un couple maximum de réponse " $M_{M}$ "

1.2.2. un couple résiduel de réponse « $M_{R}$ "

1.3 Des valeurs de ces couples sont déduites deux résistances au cisailiement non drainé du sol sur la surface de rupture considérée :

1.3.1 une résistance maximum $\tau_{M}$ correspondant au maximum de réponse,

1.3.2 une résistance résiduelle $\tau_{R}$ correspondant au couple résiduel de réponse. 

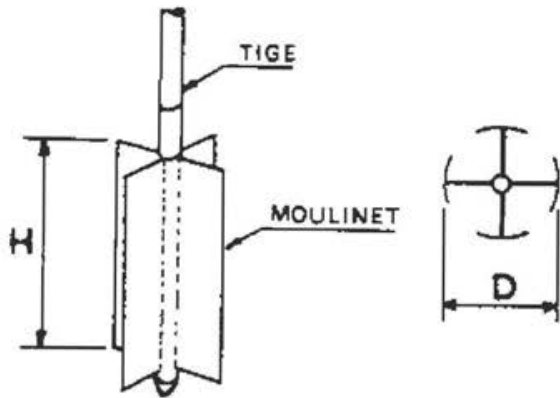

Les diametres usuels sont 60,70 , $100 \mathrm{~mm}$.

Le moulinet $\emptyset 100$ est géneralement réservé à des mesures dans des sols très mous (cohésion non drainée inférieure ou égale à 0,02 MPa).

Le petit moulinet $\emptyset 60 \mathrm{~mm}$ au contraire est utilisé dans des argiles consistantes (cohésion non drainée de l'ordre de $0,1 \mathrm{MPa}$ ).

La résistance à la pénétration du moulinet est mesurese si l'appareillage le permet.

Cette disposition vise à maintenir constantes les conditions de consolidation.

\section{Caractéristiques et limites de l'appareil}

2.1 II existe une grande variété de scissomètres qui diffèrent par la géométrie des pales, le système de train de tiges et de tubes ainsi que par le type de mise en place et le système d'application du couple.

L'entrepreneur doit faire figurer dans sa proposition les caractéristiques du scissomètre utilisé (marque, géométrie et dimensions des pales, systèmes de mesure, etc.) ainsi que l'effort de fonçage maximal de son appareil.

2.2 Le rapport de la hauteur des pales au diamètre du moulinet doit être au moins égal à 2.

2.3 Les essais au scissomètre sont conduits jusqu'à la profondeur indiquée par les documents particuliers du marché, exception faite des cas où la résistance au cisaillement du sol interdit la pénétration du moulinet.

Si un essai ne peut être réalisé à la profondeur prévue, l'entrepreneur en avise lo maître d'ouvrage, lequel prend les décisions nécessaires après consultation du géotechnicien.

\section{Procédure d'essai}

3.1 L'appareil est amené au niveau du sol à tester :

3.1.1 soit par fonçage direct,

3.1.2 soit par mise en place dans un forage préalable exécuté de telle façon que le sol n'ait pu être remanié au niveau de l'essai à réaliser.

3.2 La pénétration minimale dans le sol avant mise en rotation doit être telle que la partie supérieure du moulinet se situe à plus de $0,5 \mathrm{~m}$ en-dessous du niveau de départ du fonçage.

3.3 Cette pénétration ne doit s'accompagner d'aucune rotation.

3.4 L'installation de la tête de mesure et l'exécution de l'essai doivent être réalisées immédiatement après l'introduction du moulinet dans le sol. 
Sur certains appareils, l'influence du frottement des tiges dans le sol au-dessus du moulinet n'est pas négligeable.

A chaque changement de terrain, il convient alors de procéder prealablement à un étalonnage du frottement de ces tiges avant fixation du moulinet dans les mêmes conditions que précédemment.

II est recommandé de faire des essais tous les metres.

Un exemple de graphique de représentation est donné en annexe.

Les coordonnées du point de référence fixe doivent être rapportées au système géodésique local officiellement en vigueur.
3.5 La vitesse de rotation en tête du train de tige doit être comprise entre 6 et 18 degrés sexagésimaux par minute.

3.6 Les mesures sont faites toutes les 10 secondes et une fois le couple maximum obtenu, poursuivies pour avoir 6 lectures au-delà de ce maximum.

\section{Fréquence des essais}

La fréquence des essais, c'est-à-dire le nombre d'essais au scissomètre de chantier par forage, est fixée par le maître d'ouvrage en accord avec le géotechnicien.

\section{Dépouillement des essais}

Le dépouillement des essais et la détermination de la résistance maximum $\tau_{M}$ au cisaillement et de la résistance résiduelle $\tau_{R}$ doivent être faits par l'entreprise qui a réalisé les essais, en indiquant d'autre part les résultats des étalonnages du frottement des tiges et la résistance à la pénétration lorsqu'elle a pu être mesurée.

\section{Présentation des résultats}

Les résuitats des essais sont présentés au maître d'ouvrage sous la forme de graphiques comportant les éléments suivants :

- la désignation du chantier,

- le nom et l'adresse de l'entreprise de forage,

- la numérotation du forage et la référence du plan de situation permettant de le repérer,

- la date du début et de la fin d'exécution du forage,

- la date d'exécution des essais,

- le repérage en profondeur ot en altitude par rapport à un point de référence fixe, des renseignements portés sur le graphique,

- la description sommaire des terrains traversés,

- la ou les méthodes de perforation avec leur profondeur d'utilisation et la nature de f'outil utilisé, - le ou les diamètres du trou. 
Cette indication ne préjuge pas du niveau statique de la nappe.
- I'indication du fluide de perforation,

- les observations coricernant les arrivées d'eau et les pertes de fluide de perforation,

- l'indication des niveaux des essais au scissomètre effectués,

- à chaque niveau d'essai, les valeurs :

. de la résistance maximum au cisaillement $\tau_{\mathrm{M}}$,

. de la résistance résiduelie $\tau_{R}$,

. du couple de frottement des tiges $\sharp \mathrm{M}_{\tau}$ " si celuici a été mesuré avant l'essai,

. de la résistance à la pénétration du moulinet si celle-ci a été mesurée,

- le niveau d'eau ou de boue dans le forage en fin d'exécution.

\section{Carnet de mesures}

Si le maître d'ouvrage ou ses représentants l'exigent I'entrepreneur est tenu de fournir les carnets de mesu res que lesquels figurent en particulier les mesure: relatives au couple de frottement des tiges.

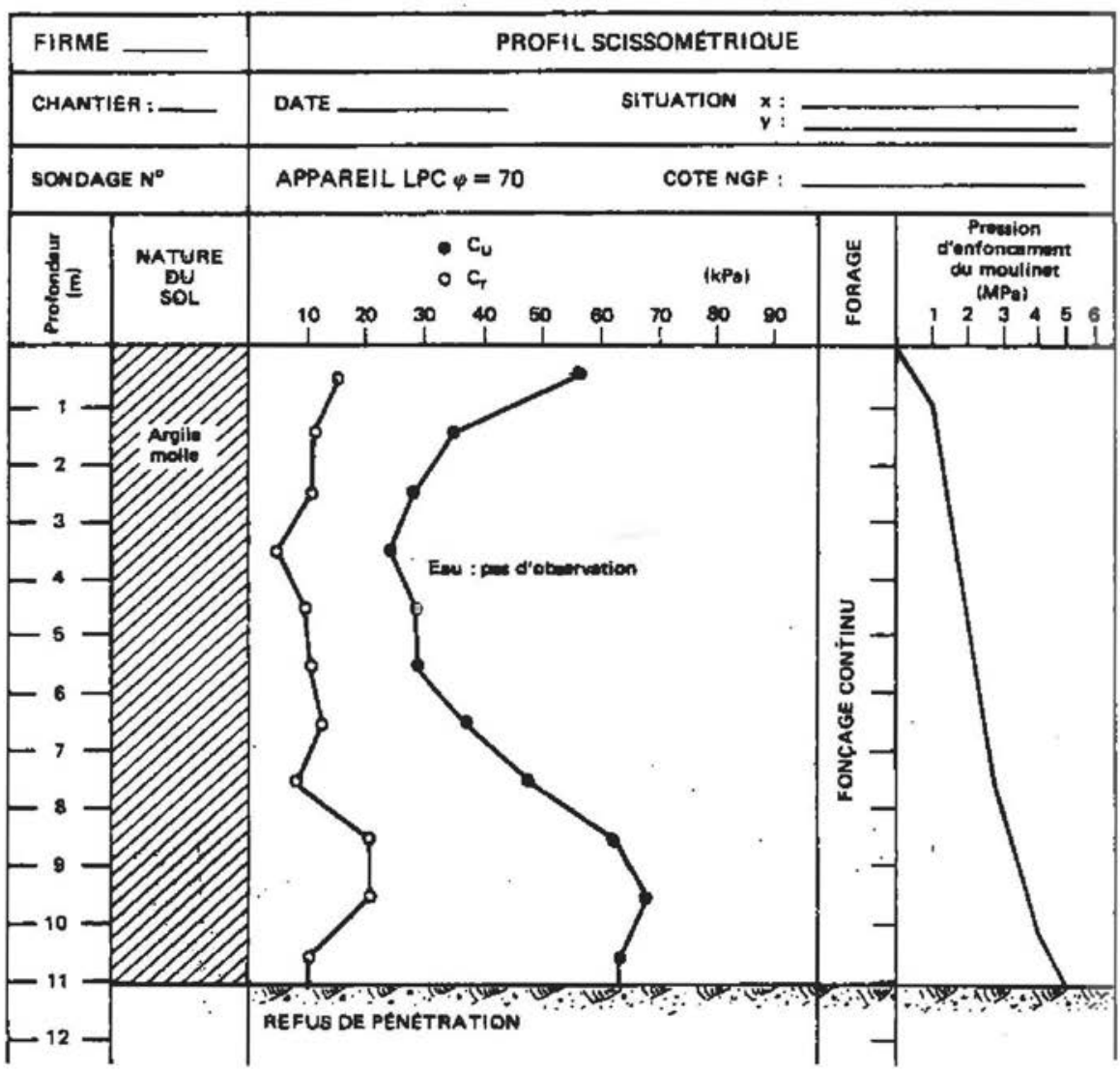




\section{G. les essais à la plaque}

Le degré de rigidité de la plaque utilisée et la position des points au droit desquels on mesure le deplacement ont une influence non négligeable sur la valeur des "modules» calculés à partir des résultats des essais de chargement surtout lorsque ces essais sont effectués sur des sols peu déformables.

Le terme aplaque rigidey désigne donc conventionnellement pour l'essai "Westergaard" un type de plaque d'une rigidité au moins équivalente à celle de la plaque decrite dans le mode opératoire en annexe et, pour l'essai de deformabilité, un type de plaque de rigidité au moins equivalente à celle de la plaque nervuré décrite par le mode opératoire CT2 du Laboratoire Central des Ponts et Chaussées.

La position des points de mesure de la deflexion est conventionnellement fixée dans la zone médiane de la plaque pour l'essai Westergaard et au centre de la plaque pour l'essai de deformabilité.

Dans le cas de l'essai de chargement, le geotechnicien tient compte, pour I'interprétation de I'essai, de la rigidité réelle de la plaque et de la position des points de mesure.

\section{Définitions}

Les essais à la plaque consistent à déterminer le déplacement vertical moyen de la surface du sol située sous une plaque rigide circulaire chargée.

Ce déplacement sous la charge correspondante est appelé déflexion et est désigné par «s».

On distingue trois types d'essais à la plaque ayant chacun un mode opératoire différent. Ce sont :

- I'essai Westergaard,

- l'essai de déformabilité,

- I'essai de chargement. 
La valeur exacte du coefficient numérique figurant au numérateur du deuxieme membre de cette formule est $6,867 \times 10^{4}$. I/ est arrondi conventionnellement à $7 \times 10^{4}$.

La distance entre les appuis du cadre et le centre de la plaque d'une part, entre les appuis du cadre et les appuis du lest d'autre part, doit être supérieured $2 \mathrm{~m}$.

On peut remplacer les 3 comparateurs par un dispositif permettant le charge ment et la mesure au centre de la plaque.

Le coefficient numérique figurant au deuxième membre de cette formule est une valeur approchée de l'expression :

$\frac{\pi}{4}\left(1-\nu^{2}\right)$

où $\nu$ est le coefficient de Poisson du sol pris égal à 0,33 dans cette approximation.

$1 /$ existe plusieurs méthodes de mesure et plusieurs modes d'interprétation de ce module de deformation à la plaque. Le plus souvent, on effectue l'essai au même point et avec la même plaque en imposant deux cycles de chargement successifs qui permettent de déterminer deux modules notés $E_{v 1}$ et $E_{v 2}$.
1.1 L'essai Westergaard est exécuté selon un mode opératoire donné en annexe. Il permet de déterminer un module conventionnel designé par $\mathrm{K}$ et appelé «module de Westergaard".

Ce module est le rapport d'une contrainte moyenne de $70 \mathrm{kPa}$ exercée sur le sol par une plaque rigide circulaire de $0,75 \pm 0,01$ mètre de diamètre, à la déflexion «s») correspondante.

$\mathrm{K}(\mathrm{Pa} / \mathrm{m})=\frac{7 \times 10^{4}(\mathrm{~Pa})}{\mathrm{s}(\mathrm{m})}$

La déflexion «s》) est mesurée à l'aide d'au moins trois comparateurs sensibles au centième de millimètre dont les palpeurs sont uniformément répartis autour de la plaque et dont les points d'attache sont fixés à un cadre indéformable prenant appui sur le sol en des points devant rester immobiles en cours d'essai.

1.2 L'essai de déformabilité à la plaque permet de déterminer un module de déformation défini conventionnellement par I'expression :

$E_{v}=0,7 \frac{q B}{s}$

où :

B est le diamètre de la plaque circulaire rigide utilisée (en m).

q est la pression moyenne appliquée sur le sol sous la surface de la plaque (en Pä),

s est la déflexion moyenne de la plaque sous la pression $\mathrm{q}(\mathrm{en} \mathrm{m}$ ).

Ce module dépend :

- du diamétre $B$ de la plaque utilisée,

- de la pression q.

- du mode opératoire suivi pour la mise en charge de la plaque.

Ces trois paramètres doivent donc être clairement explicités pour définir le module de déformation $\mathrm{E}_{\mathrm{v}}$ mesuré. 
On peut se référer au mode opératoire CT2 du Laboratoire Central des Ponts et Chaussées.

La plupart des modes opératoires existants permettent en outre, grâce à quelques aménagements et à l'usage d'une corrélation relative au diametre de la plaque utilisé, la détermination approximative du module de Westergaard.

Un minimum de 4 points de mesure alignés sur une durée maximale d'une heure est suffisant.

Si pour $q<q_{f}$ on réalise des cycles de chargement et de dechargement, on peut alors calculer un module de déformátion pour chaque cycle.

On utilise donc dans ce cas le module de Westergaard.

On utilise généralement dans ce cas les mesures faites au cours de 2 cycles de chargement successifs (modules de déformation $E_{v 1}$ et $E_{v 2}$ ).
1.3 L'essai de chargement à la plaque consiste à mesurer les déflexions «s» correspondant à une succession de pressions q appliquées au sol par la plaque suivant un programme fixé par le géotechnicien en accord avec le maître d'ouvrage.

L'essai est poursuivi :

- soit jusqu'au poinçonnement du sol,

- soit jusqu'à la limite du dispositif de réaction.

Chaque pression q est maintenue constante jusqu'à ce que la courbe représentative de la déflexion en fonction du logarithme du temps d'application soit linéaire.

L'essai permet alors de déterminer les paramètres suivants :

- le coefficient angulaire «m» de la droite traduisant pour chaque pression l'évolution de la déflexion en fonction du logarithme du temps,

- une pression critique appelée quelquefois «pression de fluagey $q_{f}$ correspondant au premier point de changement de pente de la courbe traduisant l'évolution de «m» en fonction des pressions "q",

- un module de déformation calculé pour $q<q_{f}$ par la relation :

$E_{c}=0,70 \frac{q B}{s}$

où les notations sont celles du paragraphe 1.2.

\section{Domaines d'application}

Les essais à la plaque ont essentiellement pour buts :

- soit de permettre le dimensionnement des revêtements de chaussée des pistes rigides et des dallages soumis à des charges roulantes par la méthode empirique de Westergaard,

- soit de mesurer la déformabilité des plateformes de terrassement constituées par des matériaux dont les plus gros éléments ne dépassent pas $200 \mathrm{~mm}$, 
On utilise dans ce cas l'essai de chargement.

En ce qui concerne l'auscultation d'un sol support de chaussee, de piste rigide ou de dallage soumis à des charges roulantes pour le dimensionnement de cette chaussée, de cette piste ou de ce dallage, on procède généralement au minimum a 3 essais type Westergaard et, en tout cas, à au moins un essai tous les $2000 \mathrm{~m}^{2}$ ou tous les $200 \mathrm{ml}$ selon les caractéristiques géométriques de l'ouvrage à réaliser. Cette fréquence suppose un sol homogène tant en épaisseur qu'en plan et doit donc être modifiée en cas d'hétérogénéité en décomposant la zone auscultee en "regionsy à caractéristiques homogènes.

Dans le cas de mesures de déformabilité d'une plateforme par des essais à la plaque type CT2 LCPC, les fréquences minimales d'essais sont genéralement les suivantes :

- en remblai : 3 essais au minimum et en tout cas au moins un essai tous les $1000 \mathrm{~m}^{2}$,

- en couche de forme : un essai tous les $200 \mathrm{ml}$ par couche élémentaire de compactage,

- en couche de fondation : au moins un essai tous les $2000 \mathrm{~m}^{2}$ par couche elémentaire de compactage,

- en couche de base : au moins 3 essais par couche élémentaire de compactage.

Pour l'essai Westergaard, cette plaque doit avoir un diametre de $0,75 \pm 0,01 \mathrm{~m}$. En général, cette plaque est en acier et d'une seule épaisseur; dans ce cas, elle doit avoir au minimum $25 \mathrm{~mm}$ d'épaisseur. On peut aussi realiser des empilements de plaques de $10 \mathrm{~mm}$ au moins d'épaisseur et de diamètres décroissants $0,75 \mathrm{~m}, 0,60 \mathrm{~m}, 0,45 \mathrm{~m}$ et $0,30 \mathrm{~m}$.
- soit de contrôler les fonds de fouille de fondations ou d'apporter des éléments complémentaires sur le comportement d'une fondation.

\section{Fréquence des essais}

La fréquence des essais à la plaque, c'est-à-dire le nombre d'essais à la plaque qui doivent être réalisés sur une aire déterminée en fonction du but recherché, est fixée par le maître d'ouvrage ou ses représentants sur proposition du géotechnicien.

\section{Réalisation des essais}

\subsection{Appareillage}

4.1.1 Une plaque circulaire rigide. 
Pour l'essai Westergaard, ce vérin doit pouvoir engendrer une force $d^{\prime} a u$ moins $50 \mathrm{kN}$.

Ce portique peut être simplement constitué par un profilé de 5 à $6 \mathrm{~m}$ prenant appui sous deux camions chargés, disposés parallèlement, ou encore par un seul camion de grande dimension. II est nécessaire que les roues du ou des camions soient placées a plus de $2 \mathrm{~m}$ du bord de la plaque d'essai.

Pour des essais de chargement de longue durée, il faut se prémunir des déformations dues aux variations climatiques.

On peut remplacer les trois comparateurs par un dispositif permettant le chargement et la mesure au centre de la plaque.

L'emplacement de la plaque est soigneusement débarrassé de tout matériau foisonné et réglé aussi soigneusement que possible.

Dans les terrains peu deformables, il est préférable d'utiliser le platre ou le mortier de ciment prompt.

Pour l'essai de Westergaard et pour l'essai de déformabilité, on note le temps de stabilisation de la déflexion.
4.1.2 Un vérin hydraulique, s'appuyant sur la plaque citée ci-dessus, en son centre. Ce vérin est muni d'un manomètre récemment étalonné ou, mieux, est équipé d'un dispositif dynamométrique.

4.1.3 Un portique, sous lequel le vérin prend appui par l'intermédiaire d'un dispositif formant rotule, pour appliquer la charge sur la plaque.

Les appuis au sol du lest de chargement ne doivent pas influencer les résultats de l'essai.

4.1.4 Trois ou quatre comparateurs au centième de millimètre disposés symétriquement sur la plaque dans la zone médiane de celle-ci. Un cadre indéformable approprié sert de support aux comparateurs et doit prendre appui sur le sol à deux mètres au moins du centre de la plaque et des appuis du lest de chargement.

\subsection{Préparation des essais}

4.2.1 Le sol est décapé jusqu'au niveau projeté pour l'essai. Pour assurer un bon contact du sol et de la plaque, on dispose sous la plaque une couche d'environ $1 \mathrm{~cm}$ d'épaisseur de sable fin, de plâtre, ou de mortier de ciment prompt.

4.2.2 On applique tout d'abord et pendant 30 secondes une pression de $10 \mathrm{kPa}$ sur la plaque, pour bien mettre en place tous les éléments du dispositif. Puis on supprime cette pression et dès que les comparateurs sont stabilisés, on note leurs indications. $\mathrm{C}_{\mathrm{o}}$, la moyenne de ces lectures initiales, constitue l'origine des mesures ultérieures de la déflexion.

\subsection{Réalisation de l'essai}

L'essai se fait par paliers de pression croissante seIon les indications des modes opératoires en vigueur (essai de Westergaard et essai de déformabilité), ou conformément aux indications du paragraphe 1.3.

Pour l'essai de chargement, le nombre de paliers doit être supérieur ou égal à 7. 
Un exemple d'un tel graphique est donné en annexe.

Il est souvent utile de mesurer la teneur en eau du sol à l'aplomb de la plaque.

Si la plaque n'est pas nervurée, on en précise l'épaisseur dans le cas d'une plaque unique. Dans le cas d'un empilement, on indique l'épaisseur et le diamètre de chaque plaque élémentaire.

Un exemple de chacun de ces graphiques est donné en annexe.

\section{Présentation des résultats}

5.1 Les résultats d'un essai à la plaque sont présentés sous forme d'un graphique donnant les déflexions moyennes en fonction des pressions appliquées. Ce graphique doit faire apparaître clairement les points expérimentaux correspondant à chaque palier de chargement.

5.2 Ce graphique porte en outre les indications suivantes :

- la désignation du chantier,

- le nom et l'adresse de l'entreprise ou du laboratoire qui a procédé à l'essai,

- la date à laquelle l'essai a été réalisé,

- le repérage en plan et en altitude par rapport à un repère de référence fixe du point sur lequel l'essai a été exécuté,

- la description géotechnique aussi précise que possible du sol sur lequel I'essai a été exécuté,

- le mode opératoire suivi,

- le diamètre et le type de la plaque utilisée,

- le mode de préparation de la surface du sol avant mise en place de la plaque,

- les détails notés en cours d'essai : pluie, passage d'engin lourd à proximité du point d'essai, vent, soleil, etc.

5.3 Pour l'essai de chargement, on fournit en outre les deux graphiques suivants :

- un graphique d'évolution des déflexions à pression constante en fonction du logarithme du temps qui se présente sous la forme d'un réseau de courbes d'équation :

$\mathrm{s}=\mathrm{s}_{\mathrm{o}}+\alpha \log \mathrm{t}$

- un graphique d'évolution du coefficient $\alpha$ en fonction de la pression q sous la plaque. 


\section{annexe}

\section{"l'essai Westergaard"} mode opératoire

\section{Appareillage}

1 Une plaque rigide en acier de $0,75 \mathrm{~m}$ de diamètre.

Pour une évaluation sommaire du module de Westergaard, cette plaque peut être monolithe et d'épaisseur constante pourvu que cette épaisseur soit égale ou supérieure à $25 \mathrm{~mm}$.

Pour la détermination du module en vue d'un dimensionnement de chaussées rigide ou de dallage, il est recommandé d'utiliser un empilement d'au moins quatre plaques élémentaires ayant chacune une épaisseur minimale de $10 \mathrm{~mm}$ et de diamètres décroissants $0,75-0,60-0,45$ et $0,30 \mathrm{~m}$. La différence de diamètre entre deux plaques successives ne doit en aucun cas excéder $0,15 \mathrm{~m}$.

2 Un vérin hydraulique d'une puissance minimale de 50 kN, s'appuyant sur la plaque ci-dessus en son centre et comportant une rotule sphérique.

Ce vérin doit être muni d'un manomètre récemment étalonné permettant de mesurer la pression appliquée à la plaque à $\pm 10 \mathrm{kPa}$ près ou, mieux, d'un anneau dynamométrique récemment étałonné.

3 Un portique de chargement sous lequel la rotule du vérin doit prendre appui. Les points d'appui au sol de ce portique doivent être situés à. plus de $2,00 \mathrm{~m}$ du bord de la plaque d'essai.

4 Un cadre de référence indéformable sur lequel sont fixés les comparateurs destinés à mesurer la déflexion de la plaque. Les appuis au sol de ce ca- 
dre de référence doivent être distants d'au moins $2,00 \mathrm{~m}$ des bords de la plaque et des appuis du portique.

Ce cadre doit être protégé de l'insolation et des intempéries par des dispositions appropriées.

5 Au moins trois comparateurs au $1 / 100$ de millimètre et capables d'une course de $30 \mathrm{~mm}$ disposés symétriquement sur la plaque dans la zone médiane de celle-ci.

\section{Mode opératoire}

1 Le sol est décapé jusqu'au niveau projeté pour I'essai. Pour assurer un bón contact du sol et de la plaque, on dispose, sous la plaque, une couche d'environ $1 \mathrm{~cm}$ d'épaisseur de sable fin, de plâtre ou de mortier de ciment prompt. Dans les terrains pêu déformables, il est préférable d'utiliser le plâtre ou le mortier de ciment prompt.

2 On applique une pression de $10 \mathrm{kPa}$ et on la maintient constante jusqu'à ce que la variation des indications données par les comparateurs soit inférieure à $5 / 100$ de millimètre par minute.

3 On décharge alors la plaque et dès que les comparateurs se sont stabilisés, on note leurs indications : soit $\mathrm{C}_{0}$ la moyenne de ces indications.

4 On applique alors sur la plaque une pression de $70 \mathrm{KPa}$ et on la maintient constante jusqu'à ce que la variation des indications données par les comparateurs soit inférieure à $5 / 100$ de millimètre par minute. Soit $\mathbf{C}_{1}$ les indications des comparateurs à cet instant. On a :

$$
K=\frac{7 \times 10^{4}(P a)}{C_{1}(m)-C_{0}(m)}
$$




\section{Essais à la plaque $\varnothing=600 \mathrm{~mm}$}

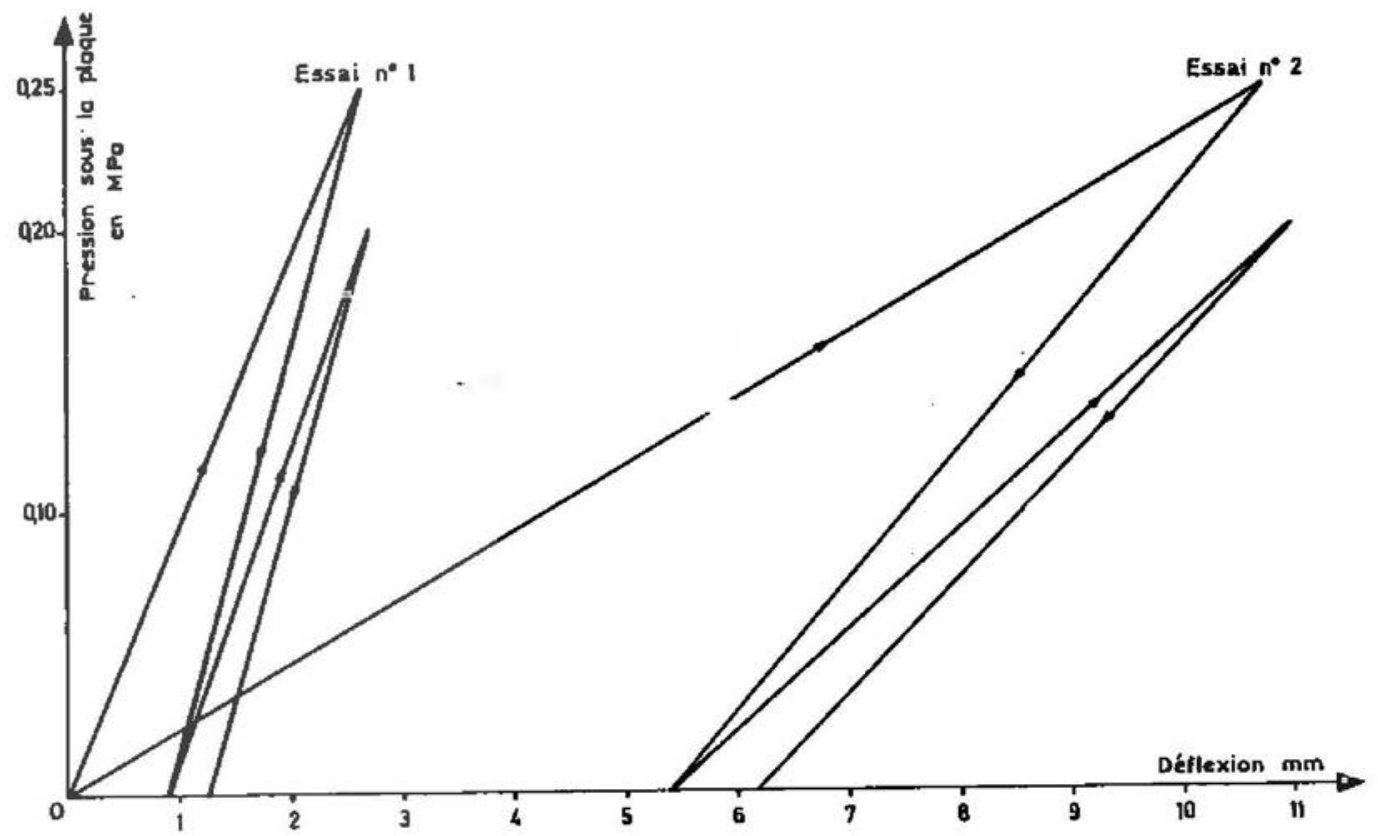




\begin{tabular}{|c|c|c|c|c|c|}
\hline \multirow{5}{*}{\multicolumn{2}{|c|}{$\begin{array}{l}\text { Essai de plaque } \mathrm{N}^{\circ} \text { : } \\
\text { Dossier } \mathrm{N}^{\circ}: 2 \\
\text { Chantier : deviation de } \\
\mathrm{S}^{\prime} \operatorname{Martin}(16) \\
\text { Opérateur: } \\
\text { Date: }\end{array}$}} & \multirow{4}{*}{\multicolumn{3}{|c|}{$\begin{array}{l}\text { Nature du matériau: Argile à silex } \\
\text { Teneur en eau sous la plaque: } 10 \% \\
\text { Teneur en eau OP: } \\
\text { Limites d'atterberg: } W_{1}=\quad I_{P=}\end{array}$}} & \multirow{5}{*}{$\begin{array}{l}\varnothing \text { de la plaque: } 600 \mathrm{~mm} \\
\text { Position de l'essai }\end{array}$} \\
\hline & & & & & \\
\hline & & & & & \\
\hline & & & & & \\
\hline & & $\begin{array}{l}\text { Valeurs } \\
\text { recommandées: }\end{array}$ & $\mathrm{Ev}_{2}>$ & $k \leqslant$ & \\
\hline \multirow{2}{*}{$\begin{array}{l}W_{1}=2,63 \mathrm{~mm} \\
W_{2}=1,56 \mathrm{~mm}\end{array}$} & \multicolumn{2}{|c|}{$\mathrm{Ev}_{1} \frac{112,5}{2,63}=62,8 \mathrm{MPa}$} & $\mathrm{Ev}_{2}$ & \multirow{2}{*}{$=1,35$} & \\
\hline & \multicolumn{2}{|c|}{$E v_{2} \frac{90,0}{1,56}=57,7 \mathrm{MPa}$} & $E v_{1}$ & & \\
\hline
\end{tabular}

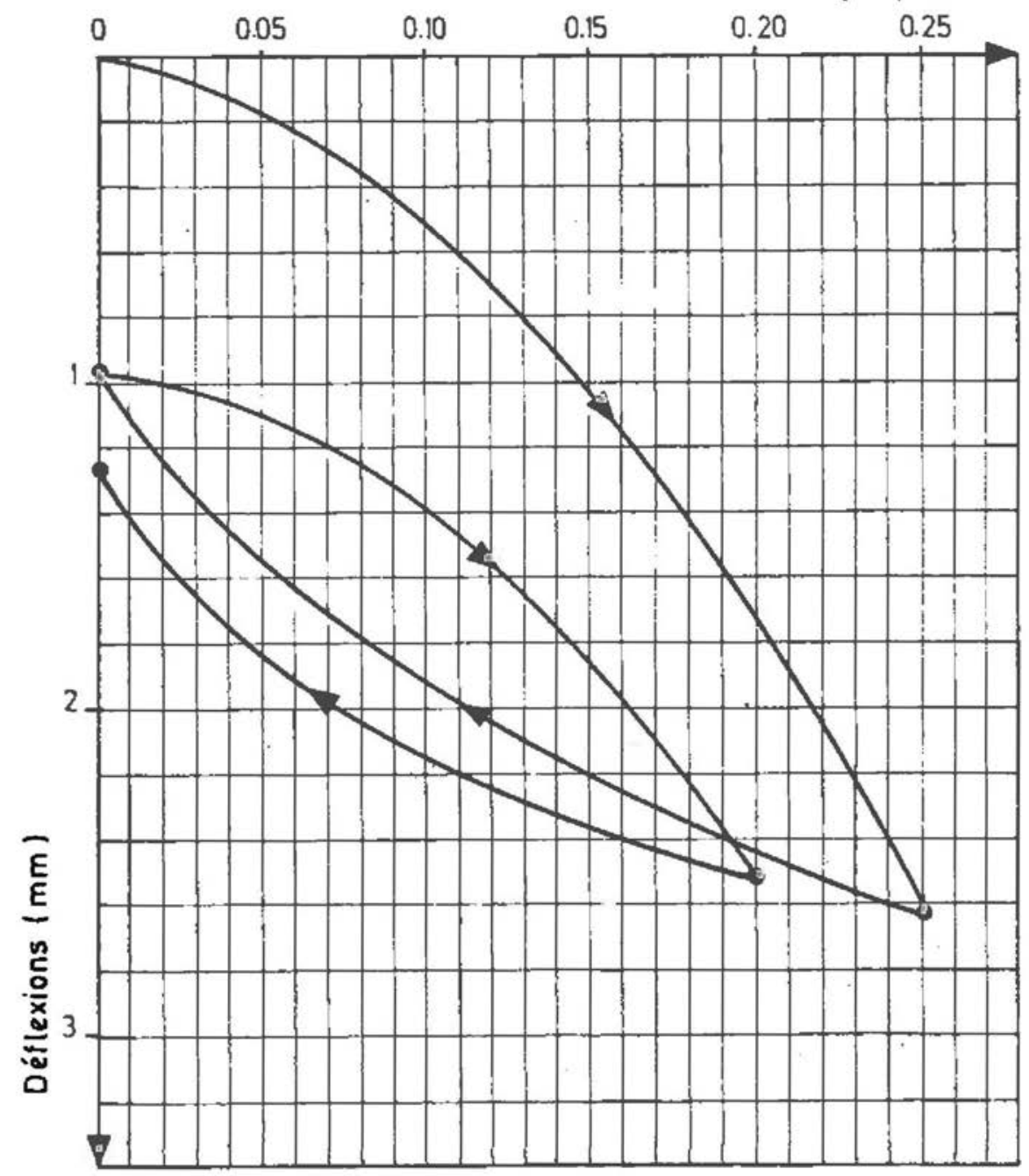

Résultat d'un essai de plaque 


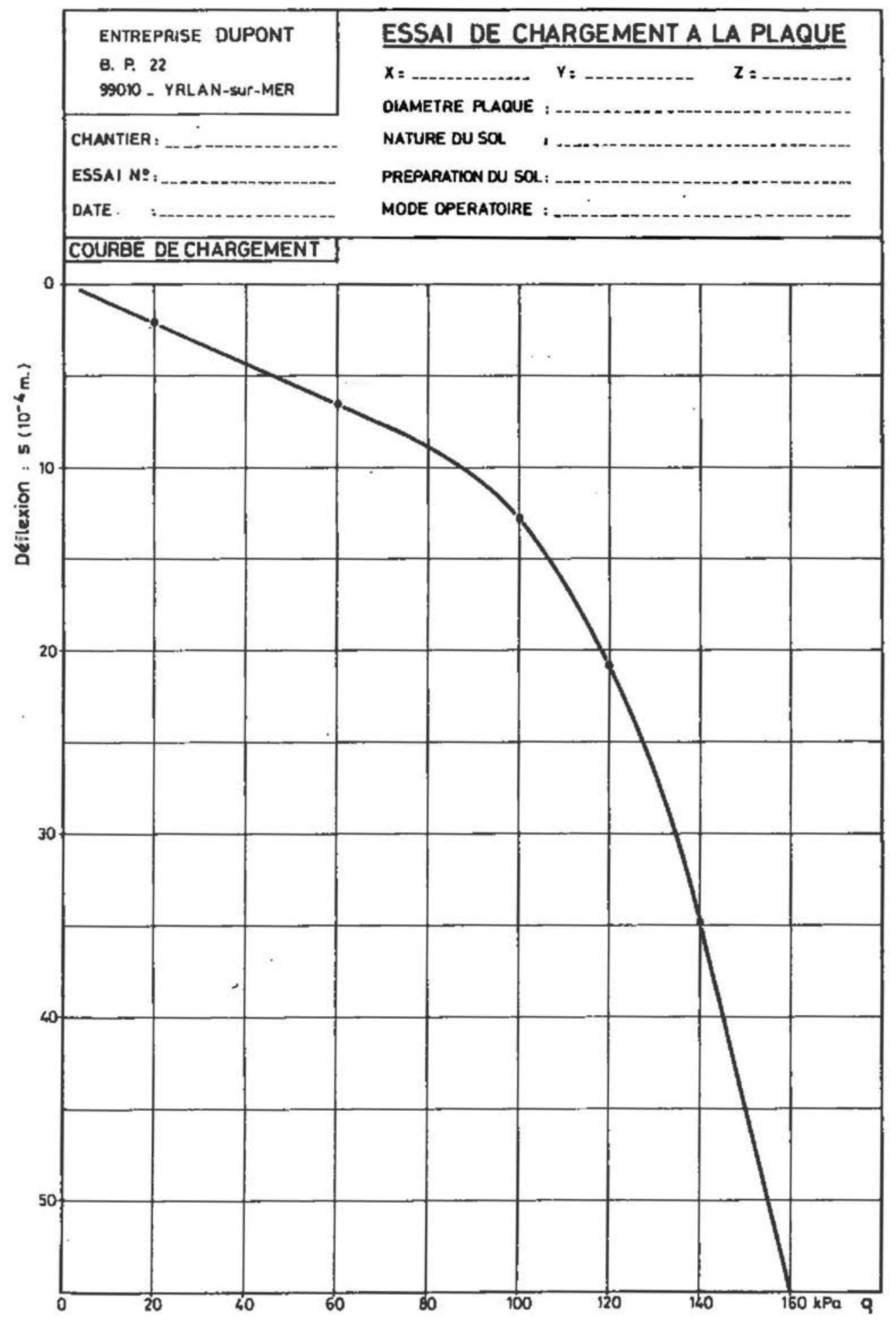




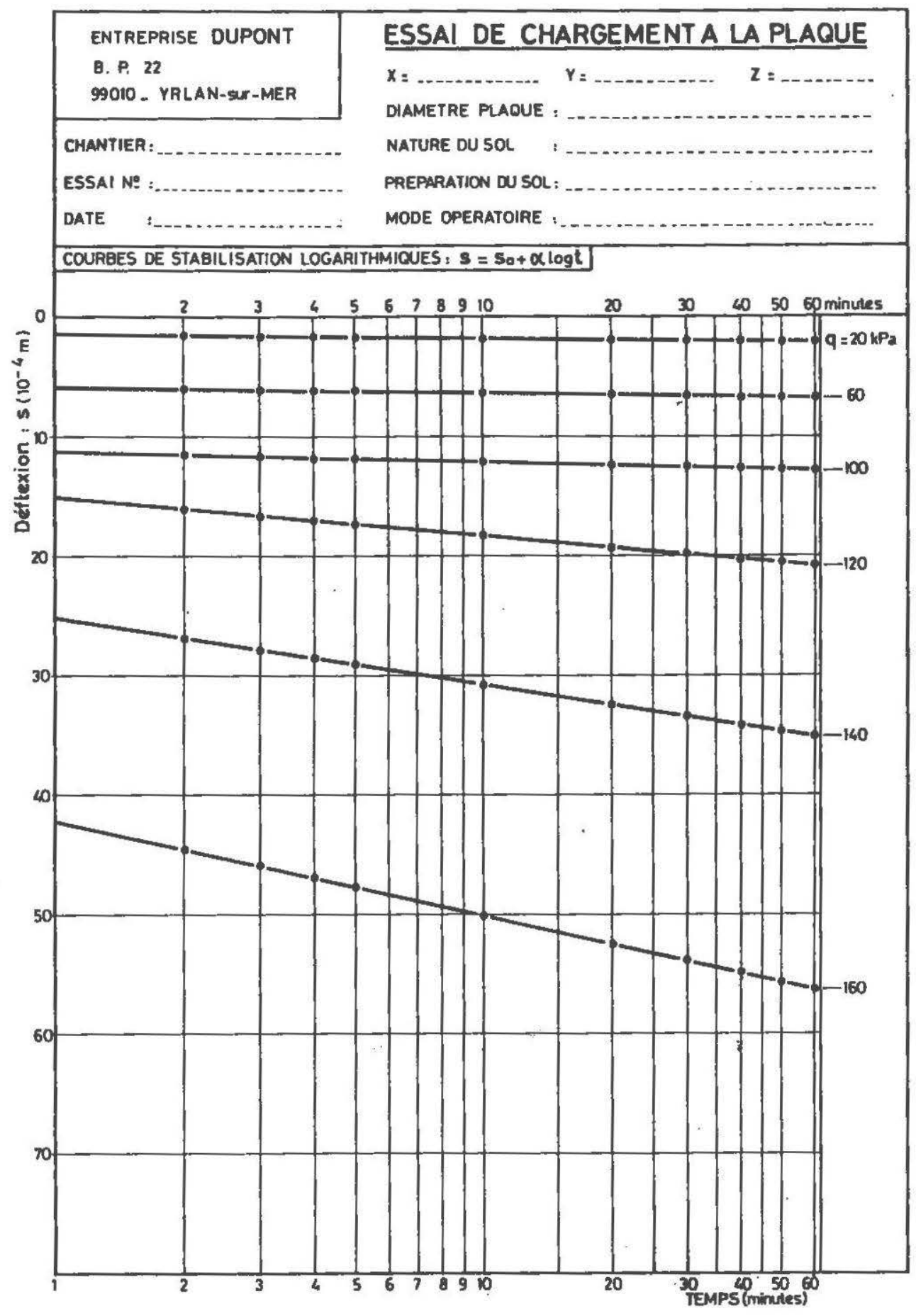




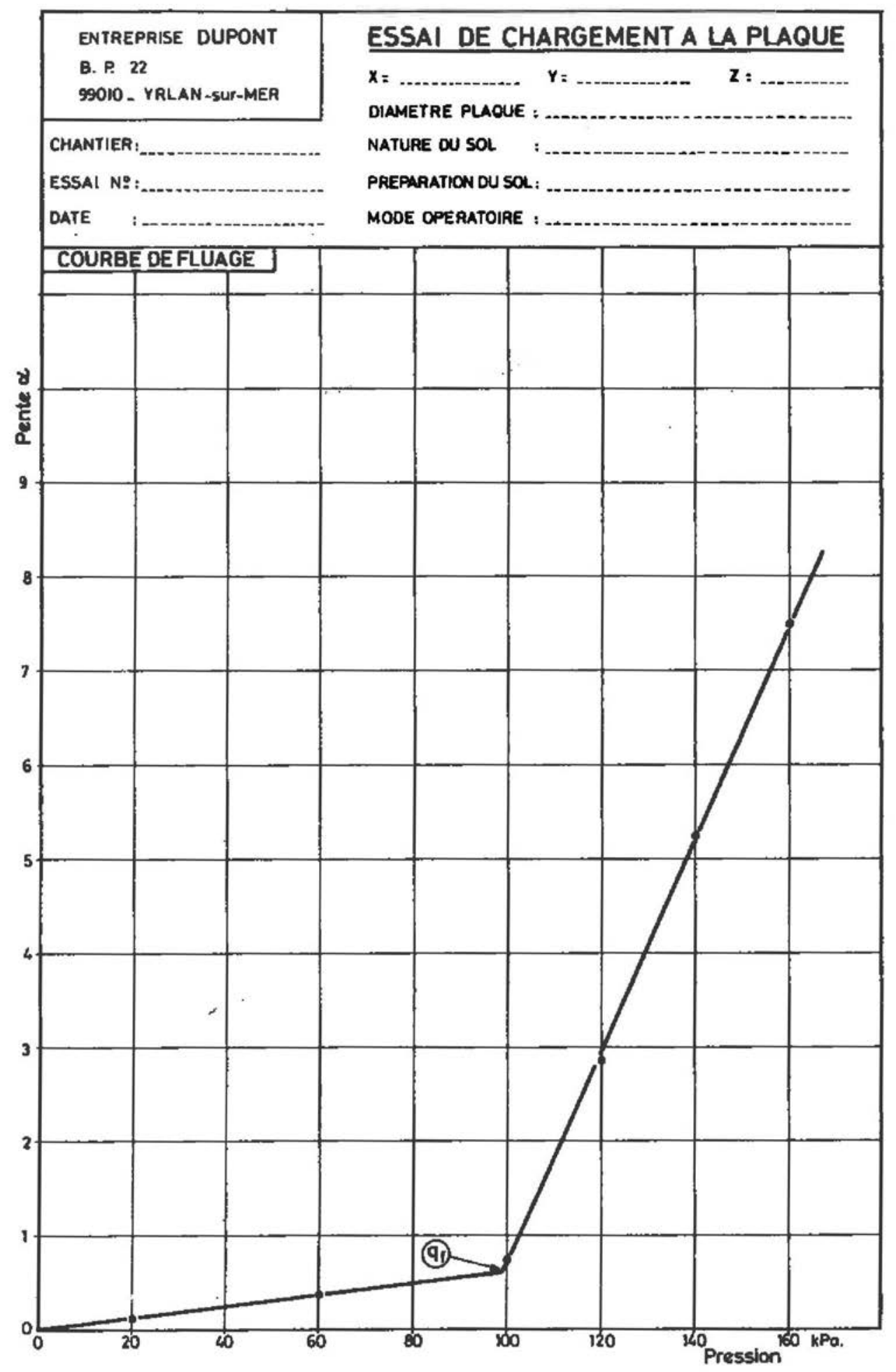




\section{H. essais d'eau dans les sondages}

Le présent document ne fixe pas de spécifications pour d'autres essais $d^{\prime} h y d r a u l i q u e$ souterraine faits à partir de sondages:

- essais ponctuels pour l'évaluation des perméabilités tels que les «caractéristiques des puits" ou micropuits et les mesures au micromoulinet,

- évaluation des transmissivités par pompages d'essai avec piezomètres, - essais de traceurs,

- prélèvements d'eau pour analyse, etc.

Le débit spécifique est le débit traversant la paroi de la poche d'essai divisé par la variation du nivéau d'eau.

Cette variation est la différence de cote entre le niveau piézométrique pendant l'essai d'eau et le niveau piézométrique naturel qui aurait été atteint au meme moment si cet essai n'avait pas été fait.

La transmissivité est le produit de l'épaisseur de l'aquifère par la valeur moyenne de sa perméabilité dans le sens de l'écoulement.

Les essais ponctuels sont souvent perturbés par le colmatage de la poche d'essai.

\section{Définitions et domaines d'application}

\subsection{Principes généraux}

Les essais d'eau dans les sondages se font :

- en isolant, par un moyen approprié, une portion du forage qui constitue alors une «cavités de forme géométrique simple, généralement appelée "poche», - puis en provoquant, par tout moyen approprié, un échange d'eau entre terrain et forage à travers les parois de la "poche», et en mesurant le débit en fonction de la pression de l'eau.

Les essais d'eau ponctuels sont conduits de façon à fournir des évaluations de la perméabilité moyenne au voisinage immédiat de la poche d'essai. Les per. méabilités apparentes se déduisent de débits spécifiques en régime à peu près stabilisé.

Les essais de transmissivité sont conduits de façon à fournir les évaluations de la transmissivité de l'aquifère avec lequel la poche d'essai communique. Les transmissivités apparentes se déduisent des variations du rabattement en fonction du temps. 
Ces essais sont intéressants pour la reconnaissance mais ne sont pas, en général, adaptés à l'évaluation précise des debits de filtration ou d'épuisement.

Pour une telle évaluation, il importe de réaliser des pompages d'essai dont les specifications sortent du cadre du présent document.

La passe d'essai a généralement quelques metres de longueur ; les surpressions vont en général jusqu'à $1 \mathrm{MPa}$.

L'essai Lugeon est un essai géotechnique, destine parfois à évaluer une perméabilité apparente à petite échelle, mais plus souvent à étudier le comportement du terrain sous de forts gradients et les possibilités d'injections de coulis d'étanchement ou de consolidation.

L'essai type Lefranc-Mandel est generalement utilisé pour mesurer, sous faible gradient, en régime permanent, la perméabilité de terrains peu cohérents.

Le rabattement spécifique est le quotient du rabattement par le débit.

Les essais de transmissivite permettent d'apprécier la transmissivité à l'échelle du rayon d'alimentation atteint en fin de pompage dans la mesure où la disposition des couches aquifères correspond aux hypothèses du modele

\subsection{Différentes sortes d'essais d'eau ponctuels}

Le présent DTU traite de trois sortes d'essais d'eau :

- I'essai type Lugeon,

- l'essai type Lefranc-Mandel,

- I'essai de transmissivité.

\subsubsection{Essai type Lugeon}

L'essai type Lugeon consiste à injecter de l'eau dans le forage, sur une hauteur définie, non tubée, successivement sous plusieurs pressions définies pendant des durées définies, et à mesurer les débits injectés dans le terrain.

L'interprétation utilise principalement des graphiques pressions-débits, où sont reportés les débits à la fin de chaque palier de pression en fonction des surpressions dans la poche d'essai par rapport à la pression d'eau naturelle.

\subsubsection{Essai type Lefranc-Mandel}

L'essai type Lefranc-Mandel consiste à modifier te niveau d'eau dans le forage, puis à mesurer :

- soit le débit en maintenant le niveau d'eau constant, - soit la variation naturelle du rabattement d'eau en fonction du temps.

La perméabilité se déduit du rabattement spécifique moyen.

Elle est interprétée en régime permanent.

En terrain peu cohérent, il faut éviter l'éboulement des parois du forage par des dispositifs appropriés.

\subsubsection{Essais de transmissivité}

Les essais de transmissivité consistent en un pornpage, à débit ou rabattement constant, suivi d'une remontée de niveau dont la durée doit être au moins aussi grande que celle du pompage.

On étudie la dérivée du rabattement spécifique en fonction du temps. 
de calcul de Theis. Une verification de ces hypotheses doit être faite on recommençant l'essai une premiere fois pendant une durée de remontée double de la précédente puis, éventuellement, une deuxième fois, on doublant encore cette durée.

Les essais d'eau ne peuvent fournir qu'un ordre de grandeur de la permeabilité ou de la transmissivite. Leur Interprétation est particulierement délicate au-dessus des nappes.

Dans ce cas :

- les conditions d'écoulement ne peuvent étre cernées avec précision,

- l'essai ne peut se faire que par injection d'eau, d'où le plus souvent un colmatage de la paroi de la upochen par des impuretés ou des bulles d'air entraînées par l'eau.

Lorsque le degre de fissuration risque de provoquer le contournement de l'obturateur, la longueur minimale d'obturation de 50 centimetres est insuffisante : il vaut mioux prévoir 1 metre, et meme dépasser cette valeur dans des cas extrémes.
Lorsqu'un teł essai est réalisé en terrain peu cohérent, il faut éviter l'éboulement des parois du forage par des dispositifs approprís.

\subsection{Domaines d'application}

Le maître d'ouvrage ou son représentant, sur proposition du géotechnicien, définit les objectifs des essais d'eau, les programmes à suivre si les terrains reconnus par les sondages sont conformes aux prévisions et la conduite à tenir en cas de faits nouveaux.

\section{Essais d'eau type Lugeon}

\subsection{Appareillage}

L'appareillage comprend :

\subsubsection{Un système d'obturation}

Le système d'obturation, utilisé pour isoler la «poche» d'eau dans le forage, doit être mis en place avec une précision de $\pm 0,1 \mathrm{~m}$. II comprend :

a) un «obturateur» destiné à limiter la «poche» à son extrémité la plus voisine de l'entrée du forage, et éventuellement un deuxième "obturateur" placé a l'autre extrémité de la "poche» si cette seconde extrémité ne correspond pas au fond du forage (voir article 2.2).

Chaque uobturateur» doit constituer un bouchon sur une hauteur de forage d'au moins $\mathbf{5 0}$ centimètres. 
En cas d'obturateur à commande mécanique, l'effort nécessaire est géné. ralement transmis par un train de tiges.

En cas d'obturateur à commande par fluide, le dispositif de manceuvre comprend, outre les tuyauteries, l'appareillage nécessaire pour mettre le fluide en pression (pompe, bouteille d'air comprimé avec détendeur, etc.).

Si l'eau est tant soit peu trouble, on risque de boucher partiellement le compteur d'eau et de colmater le terrain.

II $y$ a lieu de faire les évaluations des pertes de charge et du débit maximal par excès, lorsqu'on choisit la pompe.

En géneral, le «train de tiges» servant a manouvrer le système d'obturation est utilisé aussi comme conduite : il faut donc veiller à ce que tous les raccords soient étanches.

Les flexibles introduisent des erreurs sur les volumes d'eau, parce qu'ils sont dilatables. Ces erreurs peuvent être sensibles lorsque les volumes d'eau injectés sont faibles. b) un dispositif permettant de faire dilater radialement le ou les obturateurs, afin d'assurer leur bon contact avec les parois du forage durant tout I'essai d'eau.

c) le dispositif d'obturation doit être conçu de manière à ne pas se déplacer sous la pression d'eau introduite dans la poche d'essai.

\subsubsection{Un systẻme d'injection d'eau}

Le système d'injection d'eaú comprend :

a) une réserve d'eau très claire, ou éventuellement un branchement sur une canalisation d'alimentation en eau,

b) une pompe ou un dispositif de mise en pression présentant au moins les caractéristiques maximales suivantes fixées par le maître d'ouvrage en accord avec le géotechnicien :

- pression maximale,

- débit correspondant.

La pompe doit être munie d'un dispositif de dégàgement (bypass) comportant une vanne à fermeture progressive, afin que l'on puisse régler facilement la pression d'injection.

Entre la pompe et le dispositif de dégagement (bypass), on doit installer un accumulateur hydraulique de volume suffisant pour régulariser la pression à $\pm 5 \%$ lorsqu'on utilise une pompe à pistons.

c) les flexibles et conduites permettant d'amener l'eau jusqu'à la «poche». Ces flexibles et conduites doivent être parfaitement étanches.

\subsubsection{Un système de mesure}

Qu'il soit équipé de cadrans indicateurs, lus par un opérateur, ou d'un dispositif enregistreur, le système de mesure doit posséder les caractéristiques suivantes: 
Pour les débits atteignant ou dépassant la dizaine de litres par minute, cette précision est facilement obtenue au moyen d'un compteur d'eau volumétrique muni d'une grande aiguille.

Pour les faibles débits, on utilise comme réserve d'eau un récipient de faible section, ce qui permet de déduire le volume d'eau injecté de la baisse du niveau dans le récipient. Dans cé cas; il faut que la pompe et le circuit de dégagement ne présentent aucune fuite.

A cause de la simplicite du montage, la mesure indirecte de la pression est presque universellement adoptée.

En cas de mesure indirecte, on demande un plus grand soin dans la mesure afin de compenser, au moins partiellement, les erreurs que l'on a fait en evaluant les termes correctifs. Dans ce cas, on a intérêt à choisir le manometre de façon que la valeur maximale de sa graduation soit au plus égale a 2 fois la pression maximale prévue.

La hauteur de la poche d'essai est généralement comprise entre 3 et $5 \mathrm{~m}$.

Si on constate une perte d'eau au cours de la perforation, il peut être intéressant de procéder à un essai d'eau, méme si la passe correspondante ne mesure qu'un metre. Dans ce cas, on place l'obturateur au-delà de la zone précédemment essayée.

Les sédiments de perforation qui remontent dans le forage ont tendance à se déposer sur les parois et à les colmater. C'est pourquoi il est recommandé de faire la mesure de perméabilité dès qu'une passe de longueur suffisante est forée, avant que le colmatage soit trop fort. a) la mesure du débit doit être exacte à $\pm 3 \%$.

L'appareillage doit être étalonné à l'arrivée sur le chantier, et après tout incident en cours d'essai.

b) la mesure de la pression peut se faire soit directement dans la "poche» d'essai, soit indirectement en corrigeant de la charge d'eau et des pertes de charge une mesure faite en te̊te du forage.

La précision acceptable est :

- de $\pm 5 \%$ en cas de mesure directe,

- de $\pm 3 \%$ sur la mesure faite en tête de forage, en cas de mesure indirecte.

L'exactitude doit être vérifiée à l'arrivée de l'appareillage sur le chantier et ultérieurement après tout incident en cours d'essai. Ces vérifications se font par comparaison soit avec une "balance de pression», soit avec un "manomètre de contrôle» soumis systématiquement tous les 6 mois à la vérification d'un organisme officiel et conservé à l'abri des chocs soit par l'entrepreneur, soit par le maître d'œuvre.

\subsection{Préparation d'un essai}

\subsubsection{Hauteur d'une upoche» d'essai}

La hauteur de la poche d'essai est fixée par le maître d'ouvrage ou ses représentants sur proposition du géotechnicien.

\subsubsection{Forage}

Le forage est effectué à l'eau claire sur la hauteur de la poche d'essai et l'essai Lugeon est réalisé à l'avancement. 
En terrain de faible perméabilité, il est possible que le niveau d'eau mette plusieurs heures à se stabiliser. Dans ce cas, pour déterminer le niveau stabilisé sans attendre trop longtemps, il faut opérer comme pour un essai Lefranc, c'est-d-dire qu'il faut mesurer la romontée ou la baisse de niveau en fonction du temps et extrapoler la courbe "vitesse de remontée ou de descente en fonction de la profondeury jusqu'au point correspondant à la vitesse nulle.

\subsubsection{Nettoyage}

Le forage est nettoyé avant essai pour éliminer tous les sédiments dus à la perforation.

\subsubsection{Equipement du forage}

a) On met en place simultanément dans le forage le système d'obturation et le conduit d'injection d'eau.

Durant cette opération, il faut veiller :

- à rendre étanches les filetages des tiges utilisées pour injecter l'eau,

- à bien noter les longueurs de tous les éléments introduits dans le forage, avec une précision suffisante pour que la posițion de la "poche» d'essai soit connue à $\pm 0,1 \mathrm{~m}$.

b) Ensuite on provoque la dilatation radiale du ou des obturateurs au moyen des pressions mécaniques ou hydrauliques prévues pour assurer l'étanchéité.

c) On détermine le niveau stabilisé de la nappe au niveau de la poche.

d) Branchement de la pompe et du dispositif de mesure :

On doit avoir successivement à la sortie de la pompe :

- un accumulateur hydraulique (s'il s'agit d'une pompe à pistons),

- un dispositif de dégagement (bypass).

- une vanne précise,

- un compteur ou tout autre détecteur de volume d'eau,

- un flexible, le plus court possible,

- un manomètre.

- le train de tiges avec les obturateurs et éventuellement un détecteur de pression au niveau de la "poche» d'essai.

\subsection{Déroulement d'un essai}

2.3.1 Mesure des absorptions d'eau sous pressions croissantes

Le maître d'ouvrage ou son représentant, sur propo- 
/l s'agit de la pression d'injection lue sans correction de charge d'eau ou de perte de charge.

L'essai Lugeon proprement dit, réalises sur une passe de 3 à $5 \mathrm{~m}$, correspond aux 3 valeurs suivantes :

$P_{M}=1 \mathrm{MPa}$

$n=5$

$n^{\prime}=4$ (voir 2.3.2)

$t=10$ minutes

Si, pendant l'essai, une partie de l'eau injectée court-circuite l'obturateur et remonte dans l'espace annulaire entre train de tiges et forage, il faut essayer d'évaluer ce débit de fuite.

Parfois, un terrain absorbe de l'eau alors qu'il est réputé étanche. Dans ce cas, il existe généralement une ou plusieurs fissures dont les levres s'ouvrent sous la pression de l'aau et l'eau injecté se mot en neserve sous pression dans ces fissures. Lorsqu'on débranche ìe dispositif d'injection d'eau en fin d'essai, le forage devient artésien jusqu'à ce que les lèvres des fissures soient revenues à leur position initiale : on dit qu'il y a "contrepressiony.

Cette feuille est tenue sur le chantier à la disposition du maftre d'ouvrage et de ses representants. sition du géotechnicien, fixe une pression maximale d'essai «P $\mathrm{P}$ », un nombre «n» de paliers de pression et une durée d'injection d'eau "tw sous chacune de ces pressions.

Les valeurs des trois paramètres précédents sont fixées en fonction de la nature des terrains traversés et en fonction de la profondeur.

Pour réaliser le programme ainsi défini, on met d'abord la pompe en route, dégagement (bypass) ouvert, puis on fait monter la pression jusqu'à la valeur du premier palier ; on maintient cette pression constante pendant \&t) minutes et on mesure le volume d'eau qui, dans ces conditions̄, est absorbée par le terrain.

$\mathrm{Si}$, au début du palier, le débit n'est pas constant, on ne compte la durée de at» minutes qu'à partir du moment où il y a stabilisation du débit à $\pm 10 \%$ près.

On passe ensuite aux paliers suivants jusqu'à \&P $\mathbf{P}^{\sharp}$ si le débit maximal prévu pour la pompe choisie le permet.

\subsubsection{Mesure des absorptions d'eau sous pressions décroissantes}

Après la fin du palier à la pression $« P_{M} \rrbracket$, on fait baisser la pression par paliers. Le nombre $« n^{\prime} \|$ de ces paliers est fixé par le maître d'ouvrage out son représentant sur proposition du géotechnicien. La durée de ces paliers reste égale à «t» minutes. On mesure le volume d'eau absorbé par le terrain sous ces pressions.

En cas de "contre-pression» au débranchement du dispositif d'injection d'eau, il faut noter en fonction du temps les volumes d'eau qui sortent, ainsi que la variation éventuelle de la pression jusqu'à disparition de ce phénomène particulier.

\subsubsection{Anomalies observeres en cours d'essai}

Toute anomalie observée en cours d'essai doit être immédiatement signalée au géotechnicien.

\subsection{Feuille d'essai}

Tous les renseignements concernant l'essai sont consignés sur une feuille d'essai. Toutes les cotes y figurant doivent être rapportées à un point de référence fixe. 
Les coordonnées du point de référun. ce fixa doivent étre rattachées au sys. tème géodésique local officiellement en vigueur.
Sur cette feuille d'essai doivent figurer les éléments suivants :

\subsubsection{Le rappel des conditions de l'essai}

Tous les renseignements se rapportant aux conditions dans lesquelles a été effectué l'essai sont consignés sur la feuille d'essai, notamment :

a) la désignation du chantier et le nom et l'adresse de l'entreprise ou du laboratoire qui a procédé à l'essai,

b) la date d'exécution de l'essai,

c) le numéro d'ordre de l'essai dans le forage correspondant,

d) I'identification du forage, son inclinaison par rapport à la verticale descendante et son repérage en plan,

e) les distances entre l'origine du forage et les points suivants :

- le début et la fin de la poche d'essai,

- le fond du forage au moment de l'essai,

f) le diamètre de l'outil de forage au niveau de la poche d'essai,

g) la cote du niveau statique de la nappe,

h) la cote du manomètre,

i) les caractéristiques des conduites utilisées entre le manomètre et les obturateurs : diamètre intérieur, longueur, nombre et dimensions des raccords éventuels,

j) le type d'obturateurs utilisés, leur longueur et leur pression de blocage,

k) la nature du terrain sur la longueur de la poche d'essai,

I) un croquis résumant le montage utilisé pour l'injection d'eau et indiquant avec précision I'emplacement de la prise de pression.

\subsubsection{Le report des mesures faites}

Ces mesures se présentent :

- soit sous la forme d'un tableau donnant les pressions, les durées, les lectures du compteur d'eau, les fuites d'eau repérées dans l'espace annulaire entre conduites et parois de forage,

- soit sous la forme de graphiques enregistrés avec indication des échelles, accompagnés de toutes remarques, notamment sur leș fuites éventuelles des obturateurs.

\subsection{Présentation des résultats}

Pour chaque essai, l'entrepreneur établit un procès- 
Les pertes de charge s'évaluent à partir d'abaques tenant compte du diametre intérieur des conduites ainsi que du nombre et de la forme des raccords éventuels entre, elles. Dans le cas de très forts débits et de forages profonds, les pertes de charge ainsi évaluées peuvent représenter plus de $25 \%$ de la lecture : la précision donnée par les abaques est alors insuffisante et on doit étalonner directement l'ensemble manometre, conduites at obturateur en réalisant un montage de ces éléments, horizontalement, à la surface du sol.

Pour un chantier donné, il vaut mieux choisir une seule échelle pour les abscisses et une seule échelle pour les ordonnées, ou à la rigueur deux échelles si les résultats sont trop différents, de manière à faciliter la comparaison visuefle des résultats.

L'essai type Lugeon est le test d'absorption d'eau spécifique du rocher, c'est-à-dire d'un matériau fissure et par là même hétérogène.

En conséquence, il n'y a généralement pas de relation directe avec la perméabilité moyenne du terrain.

Dans certains cas, on peut admettre la relation très approximative suivante : 1 unité Lugeon (UL) \# $10^{-7} \mathrm{~m} / \mathrm{s}$

Lorsque l'essai est limité à des valeurs inférieures à $1 \mathrm{MPa}$, la détermination des "unités Lugeon》 se fait alors par extrapolation du diagramme de l'essai.

Cette extrapolation est très approximative car le diagramme représentatif est rarement lineaire :c'est pourquoi la représentation chiffrée en "unités Lugeon", commode pour comparer plusieurs essais entre eux, ne peut jamais se substituer entièrement au dia- verbal d'essai qu'il remet au maître de l'ouvrage ou à ses représentants. Ce procès-verbal reproduit entre autres les conditions d'essai définies en 2.4.1.

\subsubsection{Calcul des pressions vraies d'injection}

Dans le cas général où la pression n'est pas mesurée par un capteur installé dans la poche d'essai, it faut corriger les lectures de pression consignées dans la feuille d'essai. Pour cela, on tient compte des pertes de charge entre manomètre et poche d'essai ainsi que du poids de la colonne d'eau correspondante.

\subsubsection{Tracé d'un diagramme}

Après avoir fait ces corrections, on établit un diagramme représentatif en portant :

- en abscisses : les débits,

- en ordonnées : les pressions par rapport au niveau statique de la nappe.

\subsubsection{Représentation chiffrée}

A partir du diagramme précédent, on évalue le nombre d'«unités d'absorption d'eau» appelées "unités Lugeon».

Une «unité Lugeon» représente conventionnelfement l'absorption de 1 litre par minute et par mètre linéaire de longueur de la poche d'essai sous une pression de $1 \mathrm{MPa}$. 
gramme de l'essai qui doit toujours accompagner la représentation chiffrée en "unites Lugeon».

Parmi les formes de diagramme, on peut citer, en plus du diagramme linéaire, les cas suivants:

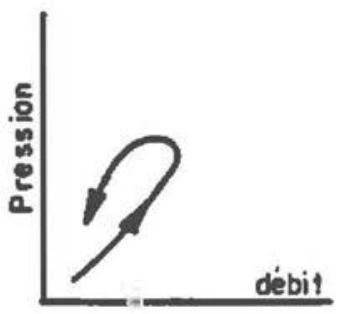

Colmatage is houte pression

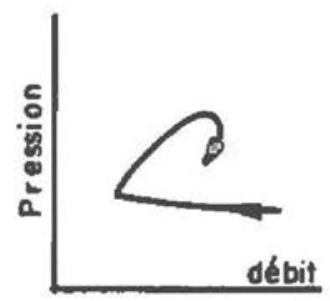

Colmofage à bosse pression Débourroge ò haute pression

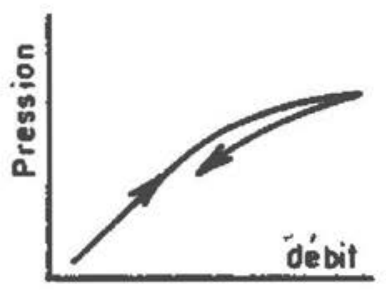

Débourroge ou ouverture des fissures

(Pression = Vobbit selon Londe ef Sabarly. Houilie blonche $N^{\circ} 1$ 1967)

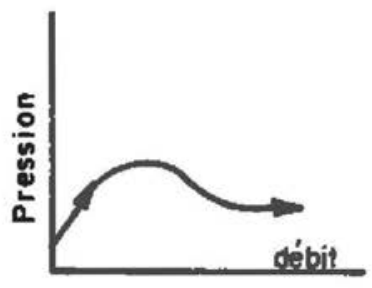

Débourrage progressif

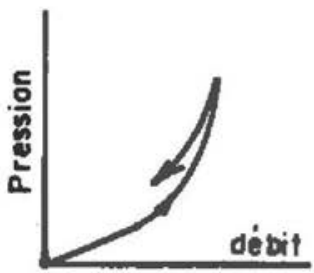

Ecoulsment furbulent 


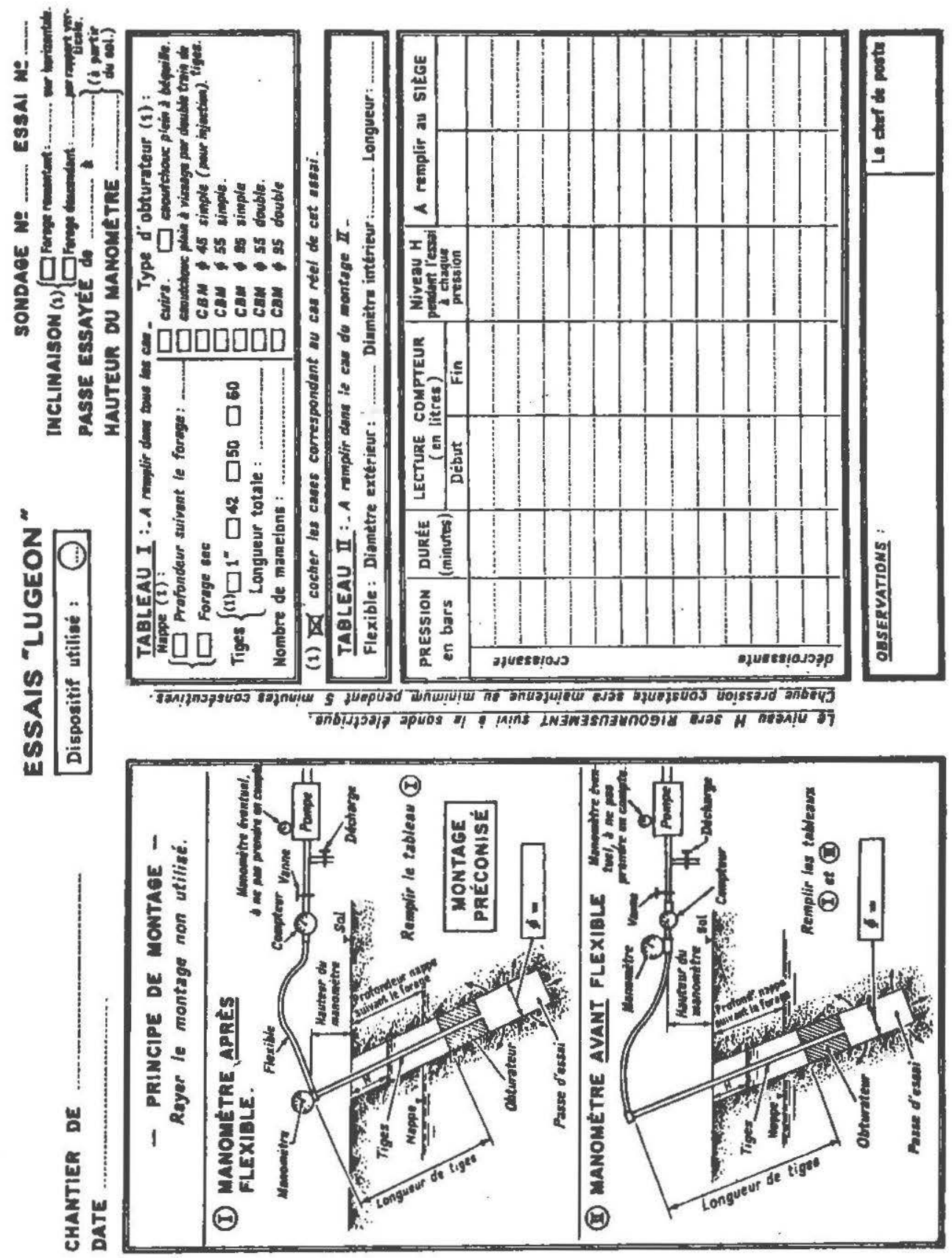


L'étancheitté doit être d'autant meilleure que la perméabilité à mesurer est plus faible. Dans le doute, il vaut mieux rechercher une étancheité parfaite des joints en garnissant les filetages d'un produit approprié.

Cette baisse peut être obtenue, suivant la perméabilité du terrain et la profondeur de la nappe, au mojen de divers procédés, par exemple :

- en utilisant une pompe dont l'aspiration est munie d'un clapet antiretour efficace,

- en utilisant un récipient de dimensions inférieures au diamètre intérieur du tubage, curette notamment,

- en soulevant rapidement un corps qui avait été auparavant immergé dans l'eau du forage.

Lorsque le forage contient une pompe, ou tout autre obstacle, on est conduit généralement à installer un tube de petit diamètre le long de la pompe pour permettre la libre descente de la sonde de détection du niveau.

Un système de mesure enregistreur peut être constitué :

- soit par un ensemble constitué de :

a) un tambour dont la rotation, en-

\section{Essais d'eau type Lefranc}

\subsection{Appareillage}

L'appareillage comprend :

3.1.1 Un système d'isolement de la "poche» d'essai.

Ce système utilise généralement les tubages qui accompagnent le matériel de perforation. II faut toutefois vérifier la bonne étanchéité des filetages lors de chaque utilisation.

3.1.2 Un système pour faire varier rapidement le niveau d'eau.

La variation rapide du niveau d'eau en début d'essai est normalement une baisse de niveau.

\subsubsection{Un système de mesure.}

Le système de mesure peut être ou non enregistreur.

\subsubsection{Système non enregistreur}

Un système non enregistreur est constitué par :

a) une sonde de détection du niveau d'eau au millimètre près, avec câble gradué à intervalies réguliers,

b) une réglette graduée en millimètres permettant d'extrapoler entre deux graduations de la sonde, la précision de la lecture étant donc de l'ordre du millimètre,

c) un chronomètre permettant des mesures à la seconde près.

\subsubsection{Système enregistreur}

En cas d'utilisation d'un système enregistreur, la précision requise pour le repérage du niveau d'eau doit être meilleure que $0,2 \mathrm{~cm}$. La mesure du temps doit pouvoir être lue à la seconde près. 
traînée par un flotteur, suit le mouvement de la surface de l'eau du forage, b) un topeur qui repère la position angulaire du tambour à intervalles de temps connus,

- soit par un ensemble constitué de :

a) une sonde multiple descendue dans le forage, capable de détecter l'arrivée de la surface de l'eau à des profondours prédéterminées (H1, H2, otc.) sous forme d'impulsions electriques transmises à la surface du sol,

b) un appareil enregistrant ces impulsions ellectriques en fonction du temps.

Si du sable fin pénetre dans le tubage, meme sur quelques centimètres seule ment, I'essai peut être entièrement faussé.

Cette étanchéité est quelquefois insuffisante dans des terrains de très faible perméabilité $\left(<10^{-6} \mathrm{~m} / \mathrm{s}\right)$ et lorsque les terrains sont suffisamment cohérents pour ne pas se resserrer élastiquement contre le tubage.

Le gravier est souvent du $5.10 \mathrm{~mm}$.

Le gravier maintient la "poche» ouverte et empéche la remontée du sa-

\subsection{Préparation d'un essai}

La préparation de la «poche» d'essai a deux objectifs distincts :

a) isoler par un tubage étanche la partie du forage qui ne doit pas être testée lors de l'essai,

b) assurer une bonne stabilité des parois du forage sur la hauteur testée, et en particulier empêcher I'entrée de sable dans le tubage.

3.2.1 Cas où un tubage est enfoncé à force au fur et à mesure du forage

La base du forage est équipée d'une trousse coupante de diamètre légèrement supérieur au diamètre extérieur du tubage dans sa partie courante, afin que le terrain se trouve comprimé au niveau de la base du tubage, assurant ainsi la meilleure étanchéité possible entre le tubage et le terrain.

- Pour confectionner une «poche» de quelques décimètres de longueur :

a) la base du tubage est enfoncée jusqu'au niveau prévu pour l'extrémité inférieure de la «poche» d'essai, le forage n'étant pas prolongé au-delà,

b) I'intérieur du tubage est nettoyé avec les outils normaux de perforation, le tubage étant maintenu plein d'eau,

c) on met alors en place, au fond du tubage, un volu. me de gravier très perméable suffisant pour constituer la poche d'essai dáfinie ci-après,

d) on remonte le tubage de quelques décimètres, en prenant soin que le sabot du tubage ne remonte pas au-dessus du gravier.

On constitue ainsi une «poche» de dimensions connues sous le sabot du tubage. 
ble. II ne fausse pas l'essai lorsque sa perméabilité est grande par rapport à celle du terrain.

On peut être obligé d'entourer le tube crépiné d'une toile filtre, ou même de deux toiles filtres superposées lune toile à mailles fines sur une toile à mailles plus grosses), si le sable à filtrer est très fin.

La longueur du tube crépiné est nettement supérieure à la longueur de la "poche» de façon que son extrémité supérieure reste toujours au-dessus d'une infiltration éventuelle de sable fin dans l'espace annulaire tube crépiné-tubage.

Dans ce cas simple où la "pochen n'est pas protégée contre un éboulement éventuel, on évite généralement que le rapport longueur de poche/diamètre du forage dépasse 10.

Ce cas est essentiellement celui des forages exécutés en rotation.

La méthode choisie doit être adaptée à la nature du terrain, à son type de fissuration, à la précision recherchée et aे l'ordre de grandeur des perméabilités.

Souvent, on utilise la méthode suivante :

a) forage arrêté au niveau du haut de la "poche» future,

b) tubage descendu a une certaine distance du fond provisoire du forage, au moins 2 fois le diamètre.
- Pour confectionner une poche de plus grande longueur:

a) la base du tubage est enfoncée jusqu'au niveau prévu pour l'extrémité inférieure de la "poche» d'es. sai, le forage n'étant pas prolongé au-delà,

b) I'intérieur du tubage est nettoyé avec les outils normaux de perforation, le tubage étant maintenu plein d'eau,

c) on met alors en place, au fond du tubage, un tube crépiné fermé à sa base, plus long que la «po. che” d'essai,

d) on remonte le tubage d'une quantité égale à la longueur de la «poche».

Si la nature du terrain est telle que ne puissent se produire sur la hauteur de la poche d'essai ni éboulement des parois du forage, ni remontée de sable, on peut réaliser une poche d'essai en nettoyant le forage immédiatement au-dessous du sabot du tubage avec les outils normaux de perforation. On doit alors vérifier, après essai, que la poche d'essai a gardé sa profondeur initiale.

3.2.2 Cas où le tubage n'est pas enfoncé à force dans le forage

Dans le cas d'un tubage descendu sans forcer dans un forage déjà exécuté en diamètre supérieur, il faut créer une étanchété entre la base de ce tubage et le terrain.

II n'existe pas de méthode imposée pour réaliser cette étanchéité, mais, compte tenu de l'importance essentielle du résultat obtenu, l'entrepreneur doit justifier I'efficacité du procédé qu'il propose, et obtenir l'accord du maître d'ouvrage. 
c) mise en place, à la base, d'un bouchon d'argile assez raide damée,

di) battage en tête du tubage pour l'enfoncer jusqu'à la base du bouchon d'argile,

e) perforation de la upochey d'essai en diametre inferieur au diametre in. térieur du tubage.

Dans beaucoup de cas, l'ajout d'eau provoque un colmatage par suite :

- soit du depot des sédiments de perforation qui se trouvent encore dans lo forage au moment de l'essai, - soit du depót des traces même imperceptibles d'argile qui se trojuvent en suspension dans l'eau utilisce,

- soít d'un dégagement de gaż dissous au contact des sables fins ou moyeins.

La méthode A ést particulièrement bien adaptée aux perméabilités inferieures a $10^{-4} \cdot \mathrm{m} / \mathrm{s}$.

Eventuallement, on installe aussi la colonine de tubes de $1^{\prime \prime}$ ou de 1,5" destinée au libre passage de la sonde de détection du niveau. .

Certains dispositifs permettent de réaliser simultainément les opérations c) et d2).

On considere que la stabilisation du niveau d'eau est atteinte lorsque la vitesse de remontise de l'eau dañ's le forage est inférieure au $1 / 20 \mathrm{dme}$ de celles mesurées au cours du début de la remontée en prenant le soín d'éliminer les premiers points de mesure s'ils sont aberrants.

Lorsque la perméabilite est très faible, la remontie complète de niveau dure tris longtemps.

On peut à la fois réduire la durée des mesures et augmenter la précision de la

\subsection{Choix de la méthode d'essai utilisée}

II existe deux méthodes d'essai, A et B, par extraction d'eau du forage et deux méthodes d'essai, $C$ et $D$, par ajout d'eau dans le forage.

Les méthodes $C$ et $D$ par ajout d'eau dans le forage ne peuvent ếtre utilisées qu'avec l'accord du maître d'ouvrage ou de ses représentants sur proposition du géotechnicien.

\subsection{Déroulement d'un essai}

\subsubsection{Méthode A par extraction d'eau du forage}

La méthode A par extraction d'eau du forage consiste :

a) à installer le système destiné à faịre varier rapidement le niveau d'eau décrit au paragraphe 3.1.2,

b) à noter le niveau statique apparent,

c) à provoquer l'abaissemient rapide du niveau de I'eau dans le forage,

d) à mesurer la remontée du niveau d'eau en fonction du temps :

d1) soit manuellement avec une précision de l'ordre du millimettre pour les profondeurs et de la seconde pour les temps. En-début de remontée, le nombre des mesures est le plus grand possible compatible avec le temps nécessaire à la lecture; les mesures peuvent ensuite être espacées progressivement lorsque la vitesse de remontée diminue, à condition que l'intervalle entre deux mesures ne dépasse pas 5 centimètres et que la fréquence de mesure reste d'au moins une toutes les minutes. Les mesures sont arrêtées lorsque le niveau d'eau est stabilisé,

d2) soit avec. un système de mesure enregistreur (voir paragraphe 3.1.3.2),

e) à recommencer une deuxième fois les opérations c) et d), si la remontée est rapide (inférieure à $15 \mathrm{mn}$ ). 
détermination de la perméabilité, de la façon suivante :

- on ne mesure la remontée que pendant $10 \mathrm{mn}$,

- on verse dans le forage une quantité d'eau propre, calculée pour faire remonter le niveau brutalement de la moitie de ce qui manque pour retrouver le niveau statique,

- on mesure la remontée durant $10 \mathrm{mn}$, - on verse dans le forage une seconde quantite d"eau propre, calculee de façon à atteindre le niveau statique à quelques décimetres près,

- on mesure la remontée durant $10 \mathrm{mn}$.

La méthode $B$ est particulièrement bien adaptée aux perméabilités supérieures aे $10^{-4} \mathrm{~m} / \mathrm{s}$.

// peut s'agir d'une pompe de surface munie d'un tuyau d'aspiration, ou d'une pompe immergée.

Eventuellement, on installe aussi dans le forage la colonne de tubes 1 " ou $1,5^{\prime \prime}$ destinée au libre passage de la sonde de detection de niveau.

La méthode $C$ est adaptée aux perméabilités inférieures à $10^{-4} \mathrm{~m} / \mathrm{s}$ pour autant qu'il ne se produise pas de colmatage.

\subsubsection{Méthode B par pompages à plusieurs débits}

La méthode B par pompages à plusieurs débits consiste :

a) à installer une pompe présentant une caractéristique "hauteur monométrique-débit» suffisante, munie d'un dispositif de dégagement (bypass) et d'une vanne, de façon que l'on puisse régler facilement le débit, avec une précision de $3 \%$,

b) à noter le niveau statique apparent,

c) à pomper un débit faible $\mathrm{Q}_{1}$, de façon que l'abaissement du niveau de l'eau dans le forage ne dépasse pas quelques décimètres, jusqu'à stabilisation du débit et du niveau et à noter débit et niveau, puis à recommencer pour des débits d'environ $2 . Q_{1}$, $3 . Q_{1}$, etc., sans que l'abaissement final du niveau de l'eau dépasse 2 mètres,

d) à noter lè niveau statique apparent après arrêt des pompages.

\subsubsection{Méthode C par ajout d'eau dans le forage}

La méthode C par ajout d'eau dans le forage consiste :

a) à noter le niveau statique apparent,

b) à verser rapidement de l'eau très claire dans le forage,

c) à mesurer la baisse du niveau en fonction du temps : 
Précision de $2 \mathrm{~mm}$ au lieu de $1 \mathrm{~mm}$ à la montée : il est plus difficile de repérer un niveau qui baisse qu'un niveau qui monte. En effet, dans le premier cas, il subsiste un ménisque d'eau au contact du détecteur de niveau méme alors que le niveau réel se trouve déjà en-dessous de ce détecteur.

On considère que la stabilisation du niveau d'eau est atteinte lorsque la vitesse de remonté de l'eau dans le forage est inférieure au 1/20ème de celles mesurées au cours du début de la remontée en prenant le soin d'eliminer les premiers points de mesure s'ils sont aberrants.

L'appréciation du colmatage ou du décolmatage peut se faire de différentes façons :

1) en cas d'enregistrement de la baisse du niveau $H$ en fonction du temps, on superpose les deux graphiques en faisant coïncider les échelles des niveaux $H$, et en décalant les échelles des temps, de façon que les deux courbes se coupent. // suffit alors de tracer de nouveaux axes par le point commun pour pouvoir comparer les deux courbes, dont les ordonnées par rapport aux nouveaux axes ne doivent pas différer de plus de $20 \%$ s'il n'y a pas colmatage,

2) en cas de mesures manuelles, si on a la possibilité de tracer immédiatement le diagramme prévu en 3.6.2, on peut admettre qu'il n'y a pas colmatage si les points représentatifs de chaque essai sont sensiblement alignés (a l'exception éventuellement des tout premiers), et si les pentes des alignements des deux essais diffèrent de moins de $20 \%$,

3) en cas de mesures manuelles, si on n'a pas la possibilité de tracer immédiatement le diagramme prévu en 3.6.2, il faut reporter les points de mesure sur un diagramme uprofondeurs en fonction $d u$ temps». On est alors ramené au cas 1). c1) soit manuellement avec une précision de l'ordre de $2 \mathrm{~mm}$ pour les profondeurs et de la seconde pour les temps. En début de descente, le nombre des mesures est le plus grand possible qui soit compatible avec le temps nécessaire à la lecture ; les mesures peuvent ensuite être espacées progressivement lorsque la vitesse de descente diminue, à condition que l'intervalle entre deux. mesures de profondeurs ne dépasse pas 5 centimètres et que la fréquence des mesures reste d'au moins une toute les minutes. Les mesures sont arrêtées lorsque le niveau d'eau est stabilisé,

c2) soit avec un système de mesure enregistreur,

d) à recommencer une deuxième fois les opérations b) et c) afin de vérifier s'il n'y a pas eu colmatage ou décolmatage, auxquels cas it y a lieu de recommencer les opérations b) et c) une troisième, puis éventuellement une quatrième fois. 
La méthode $D$ est adaptée ạux perméábilités supérieures à $5.10^{-5} \mathrm{~m} / \mathrm{s}$ pour autant qu'il ne se produise pas de colmatage.

Pour obtenir un débit de remplissage constant, on utilise généralement un bidon maintenu toujours plein d'eau par déversement et on en extrait le débit à la base par l'intermédiaire d'une vanne.

Cette feuille est tenue sur lo chantier à 'a disposition du maître d'ouvrage et de ses representants. Les coordonnées du point de référence fixe doivent étre rattachées au système géodésique local officiellemènt en vigueur.

Un exemple de feuille d'essai est donné en annexe.
3.4.4 Méthode D par alimentations à plusieurs débits

La méthode $\mathrm{D}$ par alimentations à plusieurs débits corisiste :

a) à noter le niveau statique apparent,

b) à envoyer dans le forage un débit stabilisé faible $O_{1}$ de façon que la remontée du niveau de l'eau ne dépasse pas quelques décimètres, à attendre la stabilisation du niveau et à noter débit et niveau, puis à recommencer pour des débits stabilisés d'environ 2. $Q_{1}$, $3 . Q_{1}$, etc., sans que la remonțée du niveau de l'eau dépasse 2 mètres. Ces débits doivent être mesurés à $3 \%$ près,

c) à noter le niveau statique apparent après arrêt de l'alimentation.

\subsection{Feuille d'essai}

Tous les renseignements concernant l'essai sont consignés sur une feuille d'essai.

Toutes les cotes y figurant doivent être rapportées à un point de référence fixe.

Sur cette feuille d'essai doivent figurer les éléments suivants :

\subsubsection{Le rappel des conditions de l'essai}

Tous les renseignements se rapportant aux conditions dans lesquelles a été effectué l'essai sont consignés sur la feuille d'essai, notamment :

a) la désignation du chantier et le nom et l'adresse de l'entreprise ou du laboratoire qui a procédé à I'essai,

b) la date d'exécution de l'essai,

c) le numéro d'ordre de l'essai dans le forage correspondant,

d) I'identification du forage, son inclinaison par rapport à la verticale descendante et son repérage en plan,

e) les distances entre l'origine du forage et les points suivants :

- le début et la fin de la poche d'essai,

- le fond du forage au moment de l'essai,

f) le diamètre de l'outil. de forage au niveau de la poche d'essai,

g) le diamètre intérieur du tubage utilisé,

h) la méthode de réalisation de l'étanchéité foragetubage au niveau du sabot,

i) la liste de tous les équipements descendus dans le forage avec leurs cotes principales : diamètres exté- 
La tubulure de la sonde peut constituer un tel obstacle. rieurs de la pompe ou du flexible d'aspiration, avec indication des profondeurs des changements de diamètres extérieurs et intérieurs de la colonne de tubes servant à descendre le détecteur de niveau, etcc.; - $\therefore$.

j) la cote du repère par rapport auquẹl les profondeurs d'eau sont mesurées,

k) la cote du niveau statique apparent de la nappe au début de l'essai,

I) la cote du niveau d'eau dit stabilisé en fin d'essai,

m) la nature du terrain au niveau moyen de la poche d'essai,

n) un croquis résumant le montage utilisé pour I'essai.

\subsubsection{Le report des mesures faites}

Ces mesures peuvent se présenter :

- soit sous la forme d'un tableau de chiffres, donnant les profondeurs d'eau en fonction :

. du temps (méthodes d'essai $\mathrm{A}$ et $\mathrm{C}$ ).

. du débit stabilisé (méthodes d'essai B et D),

- soit sous la forme de graphiques enregistrés avec indication des échelles, accompagnés de toutes remarques utiles,

- soit sous forme de «tops» sur une bande de papier.

\subsection{Présentation des résultats}

Pour chaque essai, I'entrepreneur établit un procèsverbal d'essai qu'il remet au maître d'ouvrage ou à ses représentants. Ce procès-verbal reproduit, entre autres, les conditions d'essai définies au paragraphe 3.5.1.

\subsubsection{Calcul des débits dans les méthodes A et C}

Dans les méthodes $A$ et $C$, les échanges d'eau entre le forage et le terrain entrainent une variation du niveau de l'eau dans le tubage.

Le débit de l'échange d'eau au temps "t» est égal au produit :

$\left(\begin{array}{c}\text { vitessse de variation } \\ \text { du niveau } \frac{\mathrm{dh}}{\mathrm{dt}}\end{array}\right) \times\left(\begin{array}{c}\begin{array}{c}\text { section intérieure } \\ \text { du tubage au niveau } \mathrm{h} \\ \text { moins la section } \\ \text { dęs obstacles }\end{array}\end{array}\right)$

\subsubsection{Tracé d'un diagramme}

On établit un diagramme représentatif en portant :

- en abscisses : les débits,

- en ordonnées vers le bass : les profondeurs d'eau. 
Principales valeurs de $\mathrm{C}$ :

Surface plane circulaire du niveau du sabot de la colonne :

$C=5,7, r$

(Donald W. Taylor)

Cavité sphérique de rayon $r$ :

$C=4 \pi \cdot r$

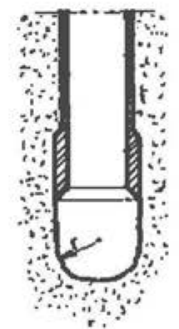

Poche cylindrique allongée :

$$
C=\frac{2 \pi \cdot d \cdot \sqrt{\frac{l^{2}}{d^{2}}-1}}{\log n \operatorname{nep}\left(\frac{1}{d}+\sqrt{\left.\frac{l^{2}}{d^{2}}-1\right)}\right.}
$$

ou plus simplement, si / est grand devant $d$ :

$C=\frac{2 \cdot \pi \cdot 1}{\text { Log.nep }\left(\frac{1}{0,67957 . d}\right)}$
(Brillant)

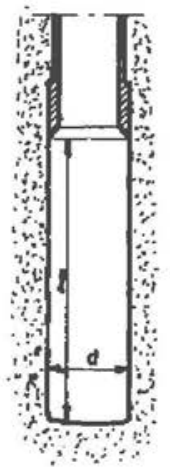

\subsubsection{Représentation chiffrée}

En partant de la loi de Darcy :

vitesse $=\mathrm{k} \times$ gradient

on démontre que l'échange d'eau qui se fait par une "poche» d'un forage est régi par une loi de la forme :

$\mathrm{k}=\frac{\mathrm{Q}}{\mathrm{C} \times \mathrm{H}}$

$k$ étant la perméabilité en $\mathrm{m} / \mathrm{s}$

$\mathrm{Q}$, le débit en $\mathrm{m}^{3} / \mathrm{s}$

$\mathrm{H}$, la charge en $\mathrm{m}$

C, un facteur, homogène à une longueur et exprimé en $\mathrm{m}$, que l'on peut caículer à partir de la loi de Darcy lorsqu'on connaît les équipotentielles et les lignes de courant de l'écoulement.

A tout diagramme du paragraphe 3.6 .2 , on peut donc faire correspondre un nombre $k$ exprimé en $\mathrm{m} / \mathrm{s}$. 


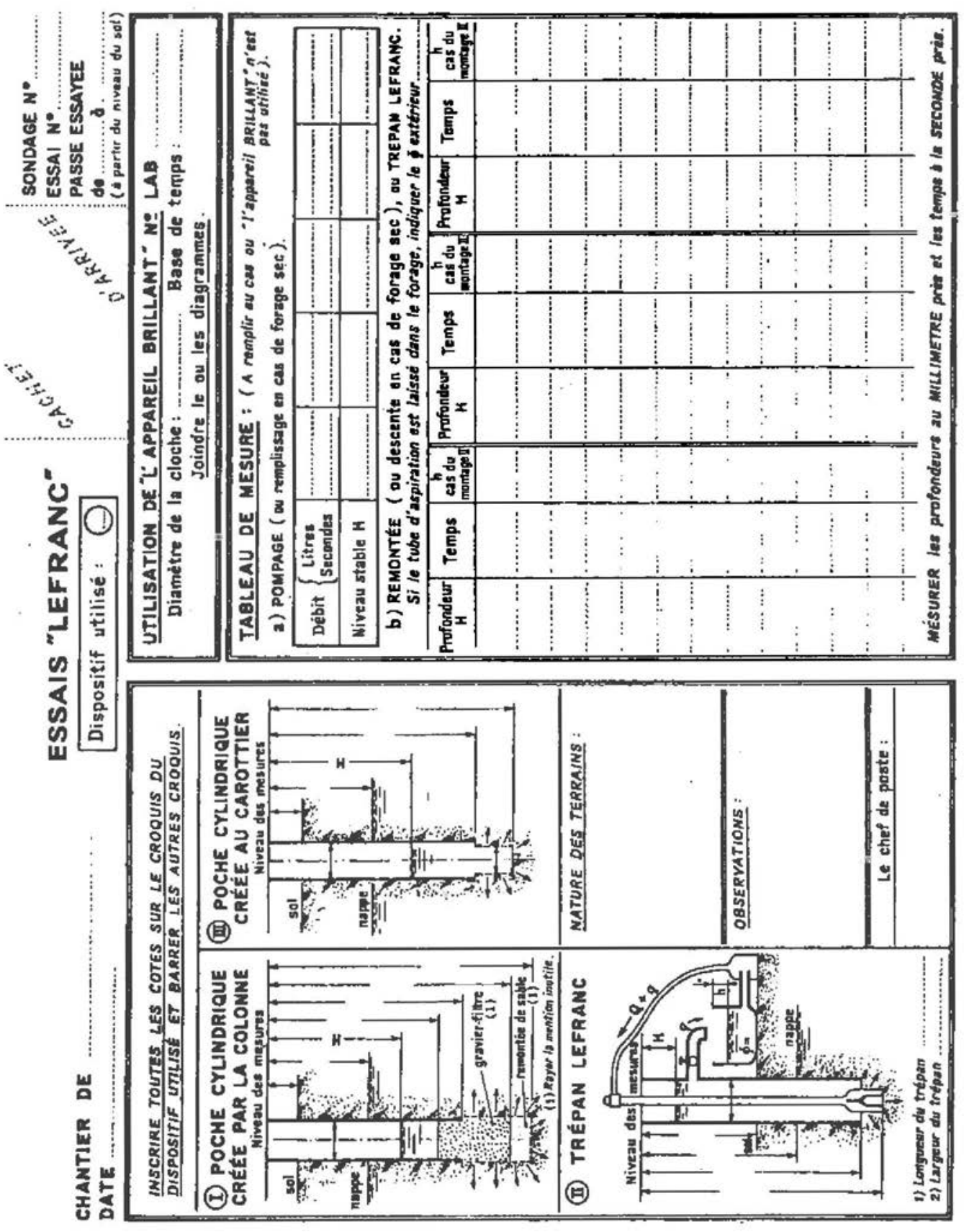


Voir 3.1.1. Des poches tres longues sont ici acceptables sauf si elles exploitent simultanément plusieurs aquifères relativement distincts et surtout un aquifère libre et un ou plusieurs aquifères captifs. Des essais LefrancMandel peuvent être nécessaires pour distinguer les aquifères, notamment par leurs niveaux piézométriques nature/s.

Les variations de densité de l'eau peuvent influencer l'interprétation des mesures de rabattement.

\section{Esşais de transmissivité}

\subsection{Appareillage}

\subsubsection{Poche d'essai}

Elle peut être réalisée comme pour un essai LefrancMandel, ou être constituée.par la prise d'alimentation d'un piézomètre ou d'un puits filtrant.

\subsubsection{Pompage ou remplissage}

L'essai peut être fait par pompage ou remplissage. Sauf accord du géotechnicien, le matériel instạllé doit permettre de pomper ou d'injecter un débit constant à $10 \%$ près.

\subsubsection{Appareils de mesure}

\subsubsection{Niṿeaux d'eau}

Les niveaux d'eau doivent être mesurés à $\pm \mathbf{2} \mathrm{mm}$ près.

\subsubsection{Dóbits}

La mesure des débits doit être exacte à $5 \%$ près. La fréquence des mesures est telle que le volume total pompé ou injecté soit connu à $10 \%$ près.

\subsubsection{Temps}

Les temps sont mesurés à la seconde près.

\subsection{Próparation d'un essai}

Le nettoyage de la epoche» ou du puits d'essai est fait par pompage jusqu'à clarification complète de l'eau extraite.

Le volume pompé doit être au moins égal au volume d'eau contenu dans le forage.

La densité des eaux pompées doit être mesurée avec une précision de $0,1 \%$.

La remontée consécutive du niveau d'eau est observée pendant une durée au moins double de celle du pompage de nettoyage.

\subsection{Premier essai}

4.3.1 La durée du pompage ou du remplissage doit satisfaire aux conditions suivantes : 
" $t_{p}$ est le retard do au volume propre du puits et s'exprime par la relation :

$t_{p}=\frac{v_{p}}{a_{F}}$

où :

$V_{p}$ est le volume d'eau contenu dans le forage entre le niveau initial et le niveau final (sans oublier l'eau contenue dans (e filtre)

et

$Q_{F}$ est le débit moyen pendant le dernier tiers de la durée do pompage ou de remplissage.

Les mesures ne sont pas significatives tant que les niveaux d'eau sont perturbés par exemple :

- par la remontée de bulles d'air après un pompage par émulsion,

- par la chute des sédiments si l'eau pompée est trouble,

- par des variations de température ou de salinité,

- par l'eau ruisselant le long du tubage après l'arrêt d'un remplissage.

Si les transmissivités déduites du 1er et du 2ème essais diffèrent de plus de $20 \%$, le maitre d'ouvrage peut demander un 3ème essai de durée encore doublée par rapport au précédent.

Cette feuille est tenue sur le chantier a la disposition du maitre d'ouvrage et de ses représentants.

Les coordonnées du point de référence fixe doivent être rattachées au systeme géodésique local officiellement en vigueur.
- être au moins égale à 5 . "t $t_{p}$, $t_{p}$ étant le retard dû au volume propre du puits,

- être au moins égale à 5-fois la durée écoulée entre la fin du pompage ou du remplissage et la première mesure significative.

4.3.2 Les niveaux d'eau après l'arrêt du pompage ou du remplissage sont suivis pendant une durée au moins double de celle du pompage ou du remplissage.

\subsection{Second essai}

4.4.1 La durée du pompage ou du remplissage est au moins le double de la durée correspondante choisie pour le premier essai.

4.4.2 Les niveaux d'eau après l'arrêt du pompage ou du remplissage sont sulvis pendant une durée au moins égale à celle du pompage ou du remplissage correspondant à cet essai.

\subsection{Feuille d'essais}

Tous les renseignements concernant les essais sont consignés sur une feuille d'essais.

Toutes les cotes y figurant doivent être rapportées à un point de référence fixe.

Sur cette feuille d'essais doivent figurer les éléments suivants : 


\subsubsection{Le rappel des conditions des essais}

Tous les renseignements se rapportant aux conditions dans lesquelles ont été effectués les essais sont consignés sur la feuille d'essais, notamment :

a) la désignation du chantier et le nom et l'adresse de l'entreprise ou du laboratoire qui a procédé aux essais,

b) la date d'exécution des essais,

c) le numéro d'ordre de chaque essai dans le forage correspondant,

d) I'identification du forage, son inclinaison par rapport à la verticale descendante et son repérage en plan,

e) les distances entre l'origine du forage et les points suivants :

- le début et la fin de la poche d'essai,

- le fond du forage au moment de l'essai,

f) le diamètre de l'outil de forage au niveau de la poche d'essai,

g) le diamètre intérieur du tubage utilisé,

h) la méthode de réalisation de l'étanchéité éventuelle forage-tubage au niveau du sabot,

i) la liste de tous les équipements descendus dans le forage avec leurs cotes principales : diamètre extérieur de la pompe ou du flexible d'aspiration, avec indication des profondeurs des changements de diamètres extérieur et intérieur de la colonne de tubes servant à descendre le détecteur de niveau, etc.,

j) la cote du repère par rapport auquel les profíndeurs d'eau sont mesurées,

k) Ia cote du niveau statique apparent de la nappe au début des essais,

1) la cote du niveau d'eau en fin d'essais,

m) la nature du terrain au niveau moyen de la poche d'essai,

n) un croquis résumant le montage utilisé pour les essais,

o) la densité des eaux de pompage ou de remplissage,

p) la turbidité des eaux en fin de pompage,

q) la présence de bulles d'air après pompage par émulsion,

r) les retombées d'eau le long des tubages après remplissage,

s) les variations naturelies de la nappe. 
Ces conventions sont destinées à rapprocher les points expérimentaux sur le graphique :

$\log \left(1+\frac{t_{F}}{t^{\prime}}\right), \| s \rrbracket$

des positions qu'ils occuperaient si le pompage ou le remplissage était fait dans les conditions correspondant au modèle de Theis : débit constant pendant la durée $t_{F}$ et volume du puits négligeable $\left(t_{p}=0\right)$.

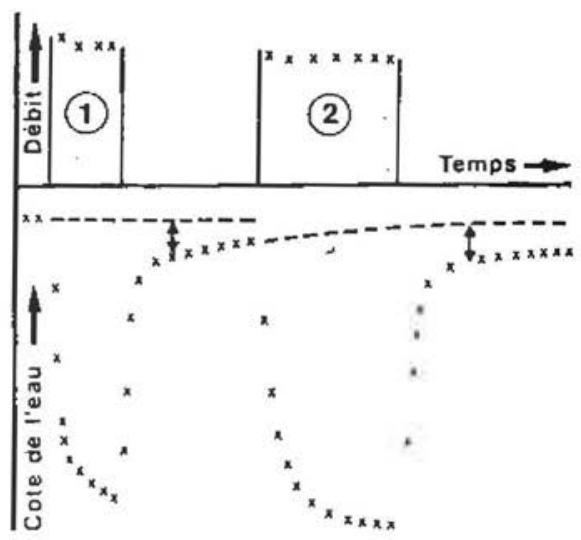

Essai de transmissivité

$s$ pendant et après le pompage 2 est calcule par rapport aux niveaux qui auraient été atteints si le pompage 2 n'avait pas été fait, extrapolés à l'aide du graphique présenté en 4.6.3.

\subsubsection{Le report des mesures faites}

Ces mesures se présentent sous la forme d'un tableau donnant les profondeurs d'eau en fonction du temps ainsi que les valeurs des débits de pompage ou de remplissage en cours d'essais.

\subsection{Présentation des résultats}

Pour chaque essai, I'entrepreneur établit un procèsverbal d'essai qu'il remet au maître d'ouvrage ou à ses représentants. Ce procès-verbal reproduit, entre autres, les conditions définies au 4.5.1.

\subsubsection{Calcul des durées équivalentes}

La durée équivalente " $\mathrm{t}_{\mathrm{F}}$ " du pompage ou du rem. plissage est le quotient du volume pompé ou injecté par le débit moyen $Q_{F}$ défini en commentaire à I'article 4.3.1.

La durée équivalente «t $t^{\prime}$ de la remontée ou de la descente du niveau d'eau après la fin du pompage ou du remplissage est comptée à partir de la fin effective du pompage ou du remplissage, puis diminuée du retard $« t_{p} »$ défini à l'article 4.3.1.

4.6.2 Calcul des remontées ou rabattements résiduels

Les rabattements résiduels (après un pompage) ou les remontées résiduelles (après un remplissage), notés «s», sont calculés par rapport au niveau qu'aurait atteint la nappe au bout du même temps à la suite de l'essai précédent.

Le croquis figurant en commentaire illustre la manière dont on doit justifier l'évaluation de ces remontées ou rabattements «résiduels». 


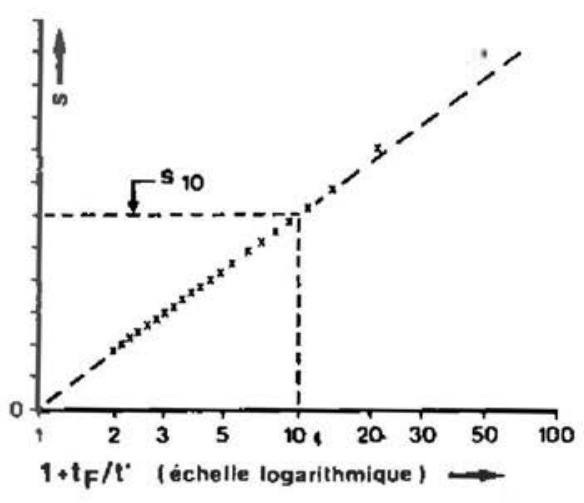

II n'est pas rare que les points expérimentaux s'ecartent nettement des courbes-type correspondant au modele de Theis.

L'entrepreneur portera sur le graphique synthetique les hypothèses que lui inspire son expérience :

- terrain hétérogène,

- aquifere multicouche,

- variations de densite, etc.

en prêtant la plus grande attention aux divergences entre les courbes relatives aux essais successifs.

\subsubsection{Tract d'un diagramme}

Les résultats finalement obtenus sont reportés sur un graphique :

$\log \left(1+\frac{t^{t}}{t^{\prime}}\right)$ en fonction de «s»

(voir ci-contre)

et résumés par une ou plusieurs transmissivités ap. parentes :

$T=0,183 \cdot \frac{Q_{F}}{s_{10}}$

$s_{10}$ étant le rabattement lu (ou la remontée lue) pour $1+\frac{t_{F}}{t^{\prime}}=10$ sur la tangente à l'origine du diagramme $(\log 1,0)$. 


\section{chapitre IV}

les méthodes géophysiques

et les essais dynamiques "in situ" 


\section{A. les méthodes géophysiques}

Ces parametres ne sont pas nécessairement liés aux caractéristiques mécaniques des sols que l'on doit utiliser pour l'étude d'un projet et leur mesure ne peut donc se substituer à la détermination de ces caractéristiques mécaniques.

\section{Définitions et domaines d'application}

Les méthodes géophysiques sont des méthodes de reconnaissance non destructives mises en cuvre à partir de la surface du sol dans le but de mesurer les valeurs et les variations de certains paramètres physiques tels que :

- résistivité électrique du sous-sol,

- intensité de la pesanteur,

- célérité des ondes de compression.

Le présent DTU examine les trois méthodes suivantes:

- méthode de reconnaissance par résistivité,

- méthode de reconnaissance par microgravimétrie,

- méthode de reconnaissance par sismique réfraction.

\section{Méthode de reconnaissance par résistivité}

Les méthodes de reconnaissance par mesures de résistivité consistent à mesurer la variation de la résistivité électrique à l'intérieur du sous-sol.

La mesure se fait au moyen d'un quadripo̊le noté $A B, M N$ constitué par quatre électrodes alignées et plantées dans le sol.

Par les électrodes $A$ et $B$, on injecte un courant continu d'intensité $I$ et on mesure la différence de potentiel $V_{M}-V_{N}$ qui apparaît entre les électrodes $M$ et $N$.

La résistivité apparente au point $O$, milieu de MN, est donnée par la formule : 
Lorsque le -sous-sol est heterogene, la résistivité apparente $\rho$ n'est pas une caractéristique du terrain.

Plusieurs phenomenes peuvent perturber les mesures :

- la polarisation spontanée,

- les courants telluriques.
Le choix du dispositif de sondage dépend du problème posé. Les dispositifs les plus couramment utilisés sont ceux dits «Schlumberger» et "Wenner".
Le choix du dispositif, de taille et de la maille du réseau de mesures nécessite l'appréhension initiale de la structure góologique du site : sondages carottés, trancheses, affleurements, etc.

Ce choix dépend de l'épaisseur de terrain que l'on veut étudier. Cette épais-
$\rho=K \cdot \frac{V_{M}-V_{N}}{1}$
où :

$\mathrm{K}$ a la dimension d'une longueur et dépend de la géométrie du dispositif,

$\rho$ est la résistivité du terrain homogène qui engendre la même différence de potentiel entre $M$ et $N$ quand on injecte la meme intensité entre $A$ et $B$.

II existe deux principales méthodes de mesure : le sondage électrique et le traîné de résistivité.

\subsection{Lo sondage électrique}

\subsubsection{Principe de la mesure}

Faire un sondage électrique en un point $O$. corisiste à mesurer la résistivité apparente du sous-sol en dón: nant au dispositif $A B M N$ centré en $O$ une taille croissante dans la même direction en conservant les proportions du dispositif.

\subsubsection{Présentation des mesures}

Les résultats d'un sondage électrique sont représentés sur un graphique à échelles bi-logarithmiques donñant les variations de la résistivité apparente en foriction dê la taille du dispositif. Sur ce graphique, sont notamment précisés :

- le point où le sondage a été effectué,

- le type de dispositif utilisé,

- l'orientation du dispositif,

- la référence aux abaques ou aux programmes de calcul utilisés pour l'interprétation.

\subsection{Le traîné de résistivité}

\subsubsection{Principe de la mesure}

Faire un traîné électrique consiste à mesurer la résistivité apparente du sous-sol en divers points de la surface du sol en conservant en taille le dispositif de mesure $A B M N$ at en l'orientant selon des directions déterminées. 
seur est déterminée en tenant compte en particulier des résultats de quelques sondages électriques préalables. On est souvent conduit à realiser plusieurs séries de trainés avec des dispositifs de dimensions différentes.
Par exemple, on peut rechercher la presence d'intercalations argileuses (conductrices) au milieu d'alluvions graveleuses (résistantes); ;́tudier l'épaisseur d'un recouvrement altéré (conducteur) sur un rocher sain (résistant).

L'efficacité de ces méthodes dépend directement du niveau' de contraste de résistivité entre les différentes natures de terrain que l'on cherche à distinguer.

La présence et la composition chimique des eaux de la nappe ont une influence importante sur les valeurs de la résistivité.

\subsubsection{Présentation des rósultats}

La présentation des résultats comprend :

- un tableau indiquant les coordonnées de chaque point de mesure et la valeur des résistivités apparentes en ce point pour chaque taille du dispositif,

- les cartes de résistivité obtenues pour les différentes tailles du dispositif.

Les hypothèses utilisées pour l'interprétation des mesures doivent étre précisées : en particulier, on doit donner les éléments ayant servi à l'étalonnage (sondages carottés, tranchées, affleurements, etc.).

\subsection{Domaine d'utilisation}

Dans. le cas où il est possible d'interpréter quantitativement un sondage électrique, on obtient une description de l'évolution de la résistivité apparente du sous-sol avec la profondeur au droit du point de mesure. Sur la base d'étalonnages, le sondage électrique peut permettre d'accéder à une connaissance schématique de la structure du sous-sol.

Par ailleurs, il permet aussi d'étalonner et de choisir les paramètres d'un dispositif de traîné de résistivité.

Le traîné de résistivité permet de déceler les variations latérales dans la structure du sous-sol dans un volume dépendant essentiellement de la taille choisie pour le dispositif de traîné.

3 Méthode de reconnaissance par microgravimétrie

La méthode de reconnaissance dite microgravimétrie consiste à mesurer, à une précision de l'ordre de 
Le résultat de la mesure :

$\Delta g=g-g_{0}$

s'exprime algébriquement en $\mu \mathrm{gal}$.

$D$ 'sutres parametres peuvent affecter les mesurẹs :

- lo relief,

- l'altitudo du point de mesure,

- la marée gravimátrique,

- la dérive de l'apparell.

L'anomalie de Bouguer apparait souvent comme la superposition de deux anomalies distinctes:

- I'anomalie négionale qui présente une variation lente et monotone et caractérise un phénomène régional,

- I'anomalie résiduelle qui caractórise I'héterogenéité locale du sous-sol.

Cette interpretation est généralement presentée sous forme cartographique.
$10^{-8} \mathrm{~m} \cdot \mathrm{s}^{-2}$, la différence " $\Delta \mathrm{g}$ » entre les valeurs «g» de la composante verticale du champ de pesanteur au droit de différents points d'un réseau à la surface du sol et la valeur "go de cette même composante au droit d'un point de référence appelé ubasen.

Le champ de pesanteur en un point est directement lié à la répartition des mạsses à l'intérieur du sol et en surface dans l'environnement de ce point.

\subsection{Principe de la reconnaissance}

La reconnaissance comporte trois phases indissociables :

- la préparation : appréciation des ordres de grandeur des anomalies présumées, estimation de l'extension en surface des zones d'anomalies, chöix de la maille de mesure, choix de la base,

- la mise en place du réseau de mesure sur le terrain : cette opération implique un relevé topographique précis en plan et en altitude,

- les mesures : chaque point du réseau doit être l'objet d'au moins trois mesures indépendantes et les retours à la base doivent être fréquents pour que les corrections soient précises.

\subsection{Présentation des résultats}

Les résultats sont consignés sur un tableau indiquant les coordonnées de chaque point de mesure et les valeurs de l'anomalie de Bouguer correspondante.

A ce tableau, doit être annexée l'interprétation des anomalies en mettant nettement en évidence les hypothéses ayant servi de base à cette interprétation.

\subsection{Domaine d'utilisation}

La microgravimétrie est souvent utilisée pour rechercher des variations latérales dans la structure du sous. sol lorsque celles-ci sont caractérisées par des changements forts et localisés de densité (cavités souterraines, tranchées, galeries, trous de bombe comblés par un remblai peu compact).

Les anomalies de pesanteur en résultant doivent être beaucoup plus importantes que les erreurs de mesure et que celles causées par les autres hétérogénéités du sous-sol et du voisinage. 
/l existe d'autres dispositifs (en croix, en $H$, en éventail, etc.). Un seul dispositif est genéralement insuffisant pour permettre I'interprétation sans ambiguitté d'une installation. Les résultats obtenus sur une méme installation à partir de plusieurs dispositifs permettent de déterminer la profondeur des differents interfaces l'aplomb de chacun des récepteurs.

Lorsque la structure du sous-sol est constituee de couches assez homogdnes avec des interfaces grossièrement plans faisant un angle faible avec la surface du sol, le diagramme obtenu pour un dispositif donné se compose de segments de droite. Les pentes des segments de droite permettent de determiner les cellérités et, a partir des ordonnées à l'origine, on peut connaître les épaisseurs des différentes couches.
Une reconnaissance microgravimétrique doit toujours être accompagnée par l'exécution d'une reconnaissance mécanique.

\section{Méthode de reconnaissance par sismique réfraction}

La méthode de reconnaissance par sismique réfraction consiste à mesurer le temps de propagation dans le sol des ondes longitudinales entre une source et des récepteurs.

La source de l'ébranlement est appelée source sismique.

On appelle installation l'implantation des récepteurs.

On appelie dispositif l'implantation de la source par rapport à l'installation.

La longueur du dispositif est la distance entre la source et le récepteur le plus éloigné.

La source constitue l'origine du dispositif.

\subsection{Principe de la reconnaissance}

Le dispositif le plus souvent utilisé est le dispositif linéaire comprenant une source et «n» récepteurs alignés.

Le premier étant installé en $A$ et le dernier en $B$, les dispositifs classiques sont :

- le tir direct : la source est placée sur la ligne de tir $A B$ au voisinage de $A$,

- le tir inverse : la source est placée sur la ligne de tir $A B$ au voisinage de $B$,

- le tir au centre : la source est placée au milieu de la ligne de tir $\mathrm{AB}$.

\subsection{Présentation des résultats}

La présentation des résultats comprend :

- une description sommaire du matériel mis en œuvre,

- un plan des dispositifs,

- pour chaque installation repérée sur le plan, le diagramme temps-distance (dromochronique) pour chacun des dispositifs avec les temps de propagation correspondant à chaque récepteur,

- les hypothèses utilisées pour l'interprétation des mesures doivent être précisées. 
Elle peut aussi contribuer à la prévision des conditions d'extraction des déblais rocheux (dóblais meubles, déblais defonçables, deblais a extraire d' l'explosif...).

\subsection{Domaine d'utilisation}

La vitesse de propagation des ondes dans un terrain dépend de sa compacité, de son état d'altération et de fracturation.

La sismique réfraction permet de déterminer les interfaces entre des formations dont les vitesses de propagation des ondes sont contrastées et croissantes avec la profondeur. Elle donne également, pour ces formations, la vitesse de propagation des ondes longitudinales.

Cette méthode est souvent mise en couvre pour déterminer l'épaisseur d'un recouvrement meuble.

Une reconnaissance par sismique réfraction doit toujours être étalonnée par l'exécution d'une reconnaissance mécanique. 


\section{B. les essais dynamiques "in situ»}

Ces essais sont mis on æuvre pour étudier le comportement dynamique des sols sous des sollicitations du type de celles engendrées par des séismes ou des machines vibrantes.

Compte tenu des faibles énergies mises en ceuvre, les déformations induites au cours de ces essais restent petites (de l'ordre de $10^{-6}$ ) et seule la valeur maximale du module de cisaillement $G$ est appréhendable. Ces méthodes ne permettent pas de determiner la valeur de l'amortissement structural des sols.

Par ailleurs, la mise en ceuvre de la méthode "crosshole" implique une tres grande précision dans le parallélisme des forages et dans la détermination du temps d'arrivée de l'onde de cisaillement.

On peut se reporter sur ce point aux publications spécialisées telles que :

- "Dynamic Geotechnical Testing», ASTM - STP 654,

- "Journal of Soil Mechanics and Foundation Division" de l'ASCE.

\section{Définitions et domaines d'application}

Les "essais dynamiques in situ» sont des essais effectués dans des forages spécialement disposés et équipés pour permettre la mesure de la vitesse de propagation des ondes de compression et de cisaillement dans chacune des formations reconnues de la masse de sol étudiée.

\section{Méthodes d'essais utilisées}

Plusieurs méthodes peuvent être utilisées selon le but recherché :

- la méthode «downhole»,

- la méthode «uphole»,

- la méthode «crosshole».

\section{Processus d'essais}

Les processus de ces essais dont l'usage est loin d'être généralisé ne sont pas encore normalisés.

II appartient donc au géotechnicien de définir le choix du dispositif à utiliser, la nature des parametres à mesurer et la précision de ces mesures. 
chapitre V

les analyses

et les essais en laboratoire 


\section{A. essais d'identification des sols}

Le mode d'exécution de certains de ces essais a fait l'objet de modes opéra. toires établis par les Laboratoires des Ponts et Chaussées. Les modes opératoires concernant les autres essais sont donnés en annexe.

On peut aussi être amené à réaliser des analyses chimiques notamment dans le cas de sols comportant des éléments agressifs ou solubles dans l'eau (teneurs en sulfates, chlorures, etc.).

L'obtention des éléments secs se fait par dessication du sol pendant 24 heures à l'étuve à $105^{\circ} \mathrm{C}\left(60^{\circ} \mathrm{C}\right.$ dans le cas des sols contenant des éléments organiques ou gypseux jusqu'à poids résidue ( constant).

La teneur en eau "d̀ saturation》 d'un sol est reliée aux poids volumiques des grains du sol et de l'eau par la relation: $w_{s a t}=\frac{\gamma_{w}}{\gamma_{d}}-\frac{\gamma_{w}}{\gamma_{s}}$

\section{Définitions}

Ces essais, généralement simples, sont destinés à décrire et à identifier les sols, à les classer et à apprécier leur état.

Le présent document décrit les essais suivants :

- mesure de la teneur en eau naturelle «W»,

- détermination des limites d'Atterberg $" W_{L} \|$

et "wp»,

- analyse granulométrique,

- détermination des poids volumiques humide " $\gamma$ ", $\sec " \boldsymbol{\gamma}_{d}$ " et du poids volumique des grains " $\boldsymbol{\gamma}_{\mathrm{s}}$,

- dosage des matières organiques «MO»,

- détermination de la teneur en carbonate de chaux " $\mathrm{CaCO}{ }_{3}$ ",

- mesure de l'équivalent de sable "ES»,

et traite du cas particulier des matériaux tourbeux.

\section{La teneur en eau naturelle «w}

Elle est définie comme le rapport en \% du poids d'eau $W_{w}$ que le sol contient. au poids $W_{d}$ de ses éléments secs.

$w \%=\frac{w_{w}}{w_{d}} \times 100$ 
où $\gamma_{w}$ est le poids volumique de l'eau.

Le degre de saturation $S_{r}$ est le rapport du volume de l'eau contenue dans un certain volume de sol au volume des vides correspondant :

$s_{r}=\frac{v_{w}}{v_{v}}$

Cette. courbe est notamment utilisée pour la classification des sols grenus. Un exemple de courbe granulometrique est donné en annexe.

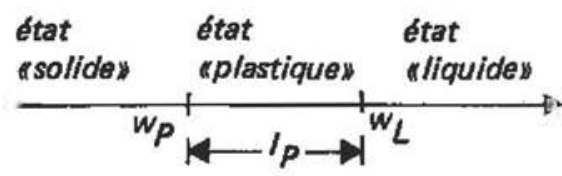

Les indices de plasticite et de consistance permettent respectivement de caractériser la plasticité et l'état d'un sol selon les tableaux ci-dessous :

\begin{tabular}{r|l}
\multicolumn{1}{l|}{$I_{P}$} & Degré de plasticite \\
\hline $0-5$ & non plastique \\
$5-15$ & peu plastique \\
$15-40$ & plastique \\
40 & très plastique
\end{tabular}

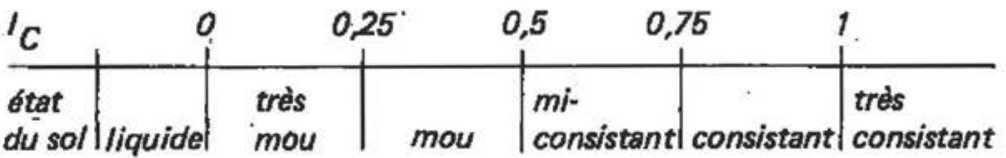

\section{L'analyse granulométrique}

Celle-ci permet de déterminer la distribution dimensionnelle en poids des éléments d'un matériau. Elle comprend deux opérations :

- tamisage pour les éléments de dimensions supérieures ou égales à $80 \mu \mathrm{m}$,

- sédimentométrie pour les éléments de dimensions inférieures à $80 \mu \mathrm{m}$.

Cette analyse est représentée par une «courbe granulométrique».

\section{Les limites d'Atterberg}

Ces limites sont les teneurs en eau caractérisant conventionnellement, sur un sol fin remanié et selon un mode opératoire bien déterminé :

a) le passage de l'état «liquide» à l'état «plastique». La teneur en eau correspondante est appelée $w_{L}$. limite de liquidité.

b) le passage de l'état «plastique» à l'état «solide». La teneur en eau correspondante est appelée $w_{p}$, limite de plasticité.

L'indice de plasticité Ip est donné par la formule suivante :

$I_{P}=w_{L}-w_{P}$

L'indice de consistance ${ }^{\mathrm{C}} \mathrm{C}$ est donné par la formule : ${ }^{\prime} C=\frac{w_{L}-w}{w_{L}-w_{P}}$

où w est la teneur en eau à l'état naturel. 


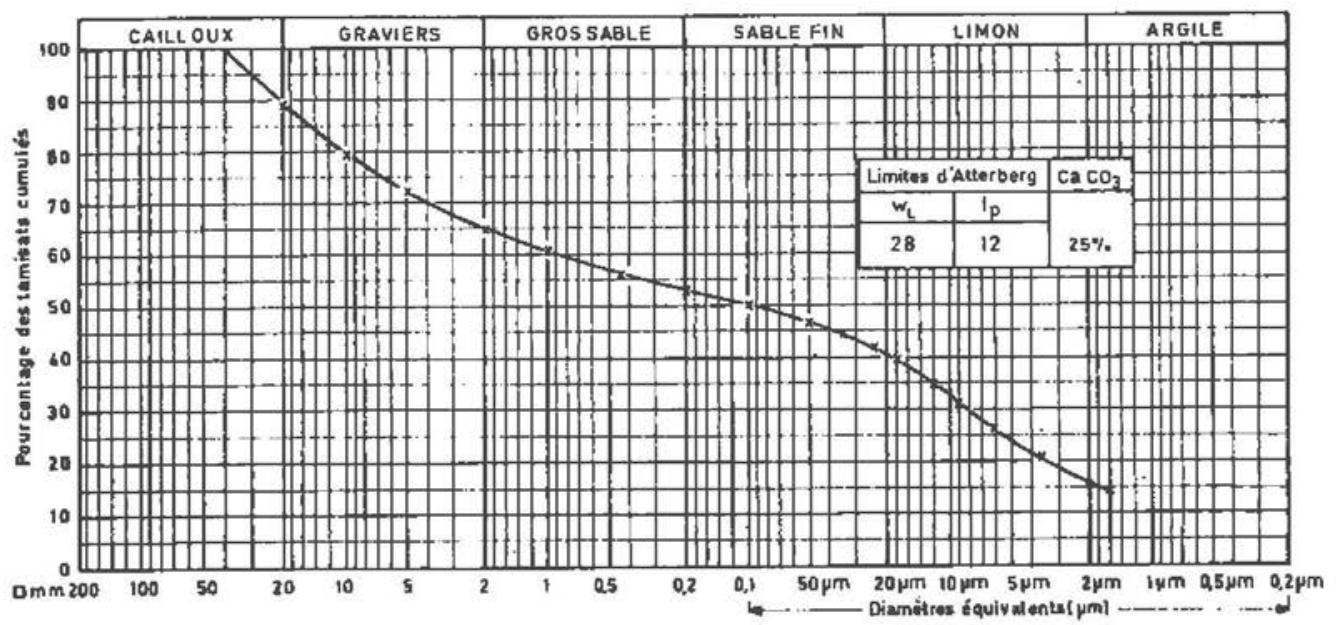

Courbe granulométrique

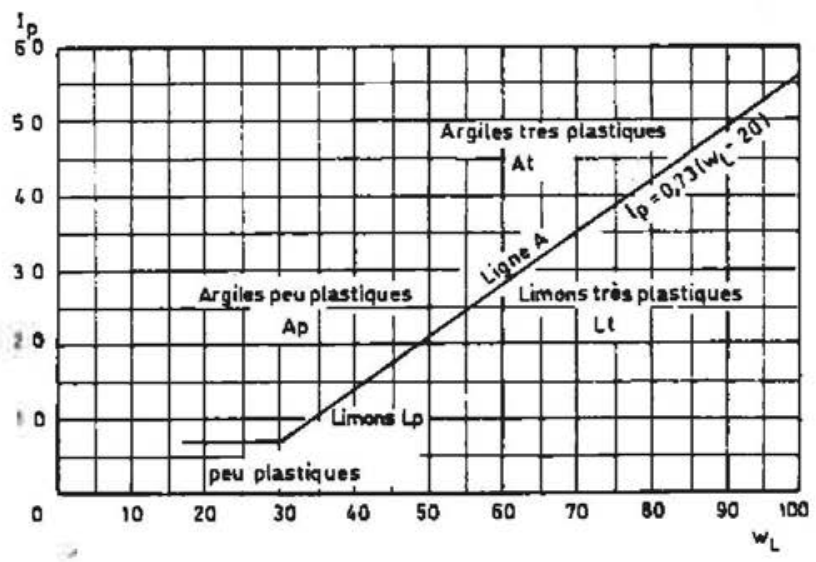

Diagramme de plasticité 
La connaissance de $w_{L}$ et de Ip permet de situer les sols fins dans un diagramme conventionnel dit «diagramme de plasticité donné en annexe.

$\gamma_{d}$ donne une indication sur la compacité de certains sols.

$\gamma, \gamma_{d}$ et $w$ sont liés par la relation

$\gamma_{d}=\frac{\gamma}{1+\frac{w \%}{100}}$

Pour les sols courants, $\gamma_{s}$ est compris entre 24 et $28 \mathrm{KN} / \mathrm{m}^{3}$.

Gypse

Montmorillonite

Orthoclase

Kaolinite

IIIite

Chlorite

Quartz

Talc

Galcite

Muscovite

Dolomite

Aragonite

Biotite

Augite

Hornblende

Limonite ou Gathite

Hématite hydratée

Magnétite

Hématite
23,2

25,6

26-30

26,6

27

27,2

27,6-31

28-29

29,4

$27,6-31$

$32 \cdot 34$

32-34

43,7

$\pm 43$

51,7

52,5

\section{Les poids volumiques}

\subsection{Le poids volumique du sol humide $\%$.}

est le rapport du poids total $W$ d'une certaine quantité de sol à son volume $\mathrm{V}$, le sol étant à une teneur en eau «w» :

$\gamma=\frac{W}{V}$

\subsection{Le poids volumique du sol sec $* \gamma_{d}$ "}

est le rapport du poids de matières sèches $W_{d}$ contenues dans le volume $V$ d'une certaine quantité de sol à une teneur en eau $w$ à ce volume $V$ :

$\gamma_{d}=\frac{W_{d}}{V}$

\subsection{Le poids volumique des grains " $\gamma_{s}$ *}

est le rapport du poids des grains $W_{d}$ contenus dans une certaine quantité de sol au volume $" V_{s} \|$ de ces grains :

$\gamma_{\mathrm{s}}=\frac{W_{\mathrm{d}}}{\mathrm{V}_{\mathrm{s}}}$

Un mode opératoire concernant la mesure de ce poids volumique est donné dans le "Manuel du laboratoire routiery de $\mathrm{M}$. Peltier, publié chez Dunod en 1955.

Cet indice de compacité est quelquefois appelé compacité relative $C_{r}$ par certains auteurs.

5.4 L'indice de densité "I D* d'un matériau pulvérulent est donné par la formule :

$I_{D}=\frac{e_{\text {max }}-e}{e_{\text {max }}-e_{\text {min }}}$ 
L'indice des vides est le rapport du volume des vides au volume total des particules solides composant le sol : $e=\frac{\gamma_{s}}{\gamma_{d}}-1$

Les appareillages et les processus d'essai sont normalisés.

\begin{tabular}{llllll}
$I_{D}$ & 0 & 0,3 & & 0,7 & 1 \\
\hline $\begin{array}{l}\text { sol } \\
\text { pulverulent láche }\end{array}$ & & & & & \\
moyen & dense
\end{tabular}

Un mode opératoire concernant cette méthode est donné dans lo "Manue/ du laboratoire routiery de $M$. Peltier publié chez Dunod en 1955.

On peut estimer le degré de décomposition "H" des matières organiques par l'essai de Von Post. Les sols organiques peuvent, selon les résultats de cet essai, être classés de la manière du schéma de la page 126.

D'après leur teneur en $\mathrm{CaCo}_{3}$, les sols fins sont classes de la manière suivante :

\begin{tabular}{c|l}
\hline$\% \mathrm{CaCo}_{3}$ & denomination \\
\hline 0 a $10 \%$ & argile ou limon \\
10 à $30 \%$ & $\begin{array}{l}\text { argile calcaire } \\
\text { ou limon calcaire } \\
30 \text { a } 70 \%\end{array}$ \\
70 à $90 \%$ & $\begin{array}{l}\text { marne } \\
\text { calcaire argileux } \\
90 \text { a } 100 \%\end{array}$ \\
\hline
\end{tabular}

Cette mesure peut être faite à l'aide du calcimetre Dietrich Frülhing. où :

$e_{\max }$ est l'indice des vides correspondant à l'état láche seion le mode opératoire utilisé,

$\mathbf{e}_{\min }$ est l'indice des vides correspondant à l'état le plus serré selon le mode opératoire utilisé,

e est l'indice des vides du sol étudié.

\section{Le dosage des matières organiques «MOk}

II n'existe pas d'essai simple et unique pour déterminer chacune des variétés de matières organiques que I'on peut rencontrer dans les sols.

Dans la pratique, seul le dosage pondéral global est réalisé par l'une des deux méthodes suivantes :

\subsection{Méthode classique}

Les matières organiques sont oxydées par un mélange de bichromate de potassium et d'acide sulfurique concentré.

\subsection{Méthode thermique}

Celle-ci fait appel à I'analyse thermique différentielle (ATD).

\section{La teneur en carbonate de calcium $" \mathrm{CaCO}_{3} \|$}

L'essai consiste ordinairement à mesurer le poids « $P$ \# de gaz carbonique $" \mathrm{CO}_{2}$ " dégagé après attaque par l'acide chlorhydrique en solution décinormale, d'une certaine quantité de sol sec.

Conventionnellement :

$\mathrm{CaCo}_{3} \%=\frac{100 \mathrm{P}}{44} \times 100$ 


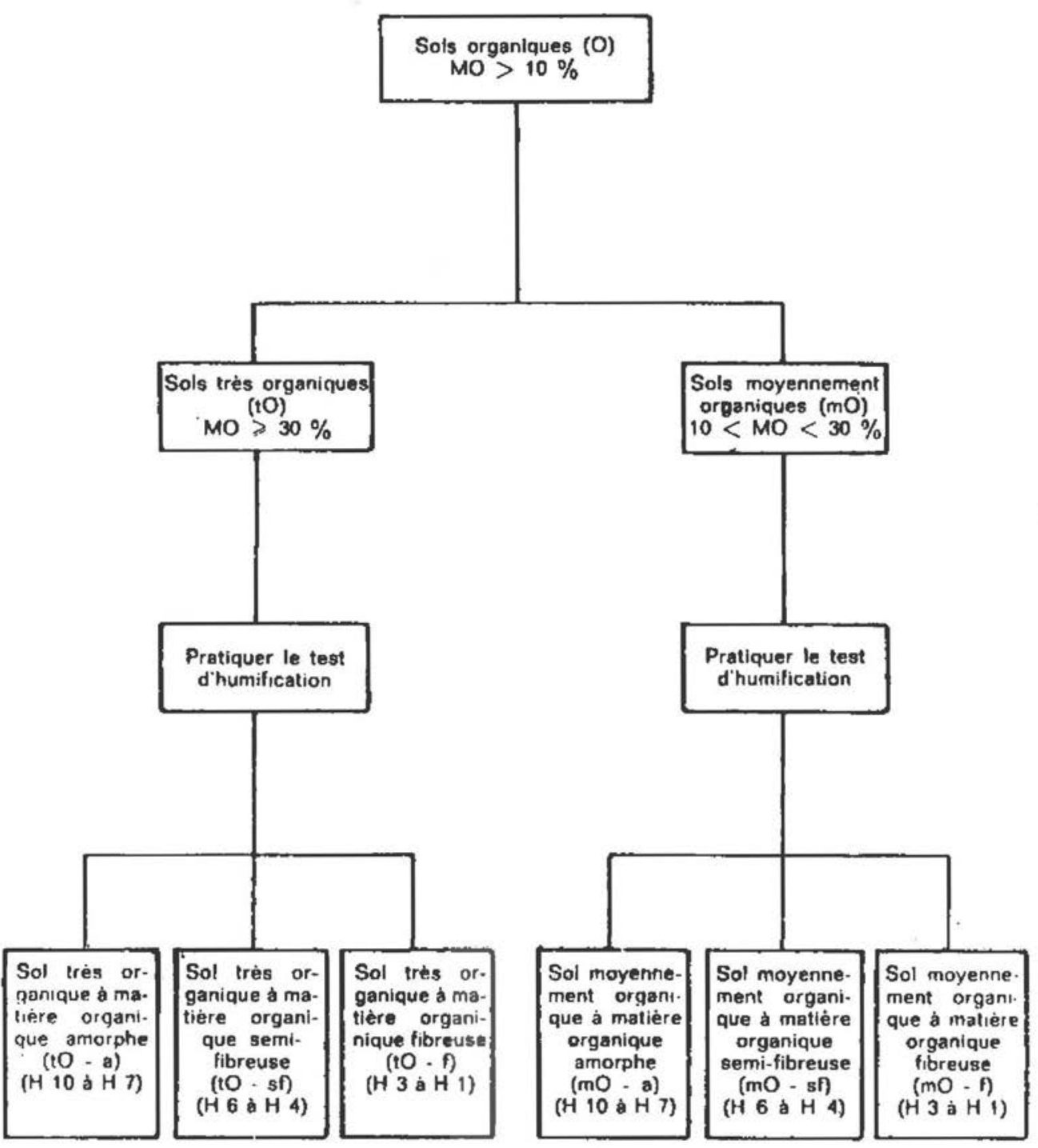


Cet essai, extrêmement sensible à la présence d'éléments fins, traduit le fait que le comportement et la perméabilite d'un sol gramulaire sont tres influences par un pourcentage, même faible, de fines.

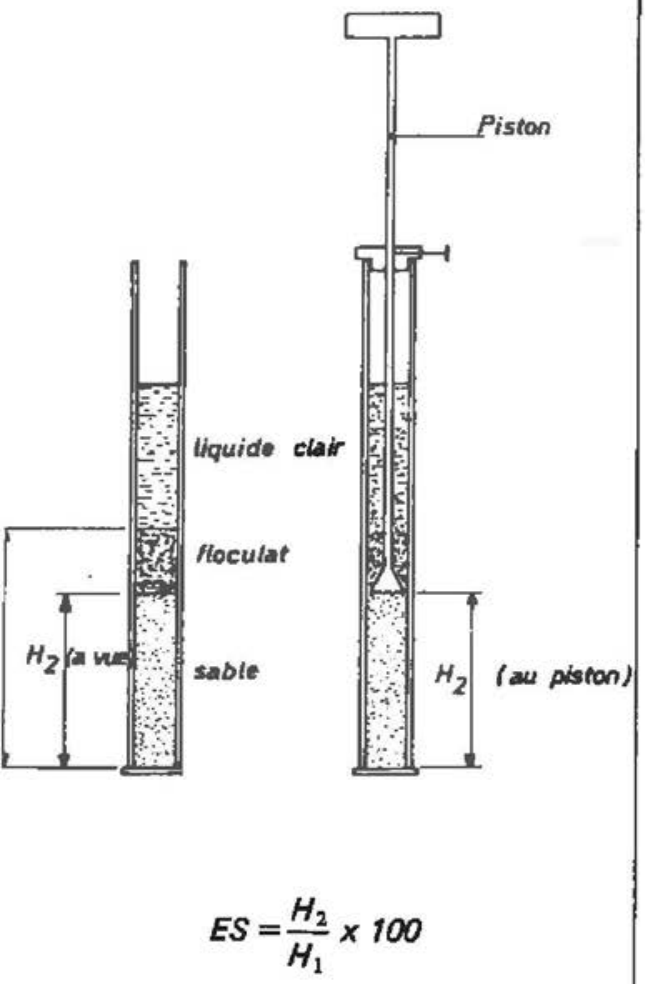

On appelle valeur de bleu,de la fraction o/d "VB o/d» la valeur de bleu correspondant à 100 grammes de la fraction granulométrique old du sol étudié.

La valeur de bleu traduit d'une façon globale la quantite et l'activité des ellements argileux contenus dans la fraction granulométrique du sol étudié. Elle est en effet liée à la surface spécifique et à l'état de charge de ces éléments.

Il existe un mode opératoire établi par le Laboratoire Central des Ponts et Chaussés pour la détermination de VB.

\section{L'équivalent de sable "ES*}

Cet essai, simple, est destiné à déterminer selon un mode opératoire précis la proportion relative d'éléments fins floculés dans la fraction d'éléments de dimensions inférieures à $\mathbf{5} \mathbf{~ m m}$ d'un sol granulaire fin. L'équivalent de sable «ES* est le rapport en \% de la hauteur de sable sédimenté à la hauteur totale du sable et du floculat.

On doit indiquer la méthode de lecture de la hauteur du sable (visuelle ou au piston).

\section{La valeur de bleu "VB»}

La valeur de bleu "VB» est la masse de bleu de méthylène nécessaire pour recouvrir d'une couche monomoléculaire de ce colorant la surface de toutes les particules de sol incluses dans une masse de 100 grammes du sol étudié. 
Les modes opératoires correspondants ne sont pas encore normalisés en France.

\section{Cas particulier des matériaux tourbeux}

Les matériaux tourbeux ne sont pas susceptibles des mêmes essais d'identification que les sols habituels.

On caractérise ces sols par :

10.1 Une teneur en aau très f́levée mesurée selon un mode opératoire bien déterminé.

10.2 Une teneur en cendres mesurée après calcination à $550^{\circ} \mathrm{C}$.

10.3 Une teneur en matiàres organiques obtenue par différence entre les résultats des deux essais précédents.

10.4 Une teneur en sable đéterminée après séparation physique au chloroforme.

10.5 Une valeur de $\mathrm{pH}$.

10.6 Un elassement granulométrique des parties non tourbeuses, fibreuses et fines.

10.7 Le volume des parties laches ou enchevetrées.

10.8 Une teneur en air.

10.9 Les capacités de rétention en eau et en air. 


\section{B. essais de résistance mécanique}

II existe pour chacun de ces deux essais un mode opératoire du Laboratoi. re Central des Ponts et Chaussées.
Les dimensions usuelles des sections horizontales de cette boîte sont $6 \times 6 \mathrm{~cm}$ ou $10 \times 10 \mathrm{~cm}$ ou bien en. core $\emptyset 6 \mathrm{~cm}$ ou $\emptyset 10 \mathrm{~cm}$ dans le cas d'une boîte circulaire.

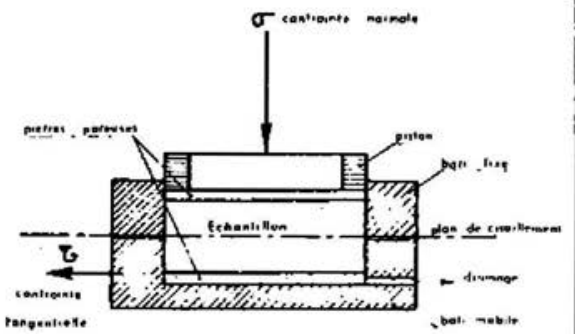

Schéma de la boite de cisaillement

\section{Définitions}

Les essais de résistance mécanique ont pour objet de déterminer la résistance des sols au cisaillement.

Les trois essais les plus utilisés sont :

- l'essai de cisaillement rectiligne à la boîte de Casagrande, - l'essai de compression triaxiale, - I'essai de compression simple.

\section{L'essai de cisaillement rectiligne}

\subsection{Principe de I'essai}

L'échantillon de sol est placé dans une boîte compo. sée de deux parties mobiles qui peuvent se déplacer horizontalement l'une par rapport à l'autre selon une direction déterminée.

Un piston permet d'exercer sur l'échantillon une contrainte verticale donnée $\sigma$.

L'échantillon est cisaillé à vitesse constante suivant un plan imposé.

\subsection{Différents types d'essai}

Dans le cas général, l'essai comporte deux phases :

- une phase préliminaire dite de consolidation.

Au cours de celle-ci, la contrainte verticale est appliquée, en présence d'eau, jusqu'à stabilisation.

L'essai est alors dit "consolidé». Si cette phase n'est pas réalisée, l'essai est alors dit «non consolidé». 
Dans cet essai, on ne maîtrise pas les conditions de drainage dans le plan de cisaillement. II y a donc lieu, surtout pour les sols fins, d'être très prudent dans son interprétation.

Dans les argiles pratiquement imperméables, l'essai rapide peut étre considén comme "non drainé».

Dans les sables propres, l'essai peut étre considén comme "drainê dans tous les cas.

Dans les sols intermédiaires, il $y$ a généralement un drainage partiel dans le plan de cisaillement même au cours d'un essai rapide.

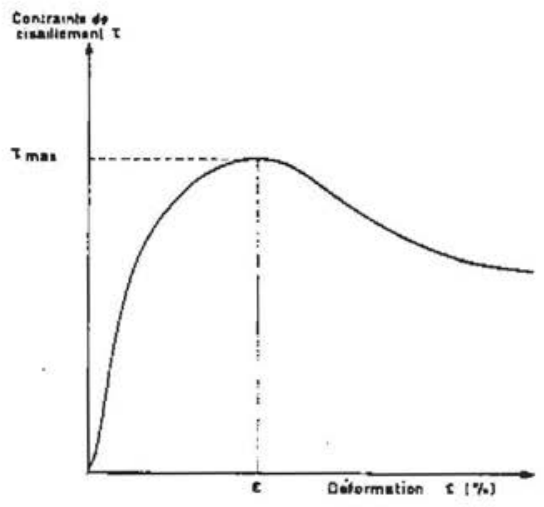

Courbe de résistance au cisaillement

Lorsque la résistance au cisaillement à la rupture correspond au maximum de la courbe contrainte-déplacement, on peut également distinguer une résistance dite "de palier» généralement atteinte pour une deformation relative de l'ordre de $20 \%$.
- une phase dite de cisaillement qui peut se faire : . soit à grande vitesse de déplacement et sans dis: positif de drainage : I'essai est alors dit rapide,

. soit à vitesse de déplacement lente, I'appareil étant muni de son dispositif de drainage : I'essai est alors dit lent.

On distingue donc habituellement trois types d'essais :

- I'essai non consolidé rapide,

- I'essai consolidé rapide.

- I'essai consolidé lent.

\subsection{Vitesses d'essai}

L'ordre de grandeur des vitesses d'essai est le suivant :

- essai rapide : 1,5 à $2 \mathrm{~mm} / \mathrm{minute,}$

- essai lent : $<5 \mu \mathrm{m} /$ minute.

\subsection{Résistance au cisaiłlement}

Pendant I'essai, on note l'évolution de la contrainte de cisaillement $\tau$ en fonction du déplacement.

La résistance au cisaillement "à la rupture» est, conventionnellement, la résistance au cisaillement maximale obtenue pendant l'essai.

Lorsque la courbe contrainte-déplacement ne présente pas de maximum bien caractérisé, la résistance au cisaillement «à la rupture» est conventionnellement la résistance au cisailiement correspondant à un déplacement égal au $\uparrow / 10$ ème du côté ou du diamètre de la boîte. 
Cette resistance est distincte de la résistance dite "de palier". Ces essais permettent de simuler de grands déplacements le long d'une surface de rupture.

Les parametres "cohésion» et "angle de frottement interney dépendent $d u$ type d'essai et des conditions de drainage.

Pour les sols fins, le diametre usuel de l'éprouvette est de 35 à $40 \mathrm{~mm}$ ét l'élancement usuel est de 2,5.

L'éprouvette est isolée du fluide par une fine membrane souple et etanche.

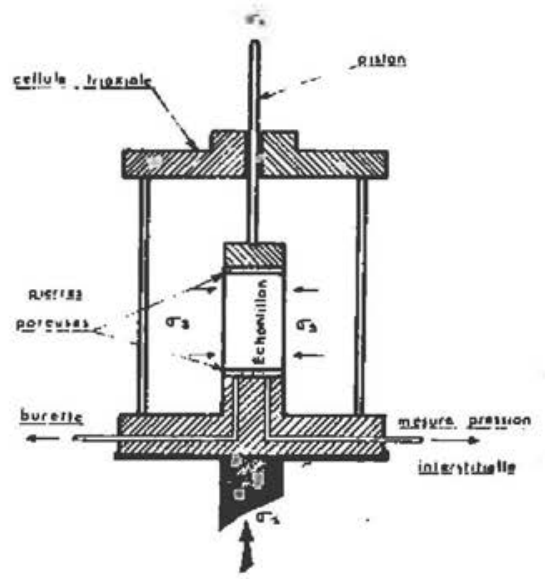

Schéma de la cellule triaxiale

On appelle "déviateur de contrainte» la différence $\sigma_{1}-\sigma_{3}$.

\subsection{Résistance résiduelle}

On appelle résistance résiduelle d'un' sol fin la résistance au cisaillement plan mesurée à l'aide d'essais de cisaillement spéciaux.

\subsection{Critère de rupture}

En répétant au moins trois fois l'essai avec des valeurs différentes de la contrainte verticale normale $\sigma$, on peut déterminer un critère de rupture en termes de cohésion et d'angle de frottement interne.

\section{L'essai triaxial}

\subsection{Principe de l'essai}

L'essai consiste à soumettre une éprouvette cylindrique de sol, d'élancement compris entre 2 et 2,6, à :

- une pression hydrostatique $\sigma_{3}$ appelée «étreinte latérale», appliquée par l'intermédiaire d'un fluide remplissant la cellule de I'appareil,

- une contrainte axiale $\sigma_{1}$ appliquée par l'intermédiaire d'un piston.

L'essai courant consiste à faire croître $\sigma_{1}$ à vitesse de déformation constante jusqu'à la rupture de l'éprouvette, $\sigma_{3}$ restant constante.

Pour les sols grenus, le diamètre de l'éprouvette est au moins égal à 5 fois la dimension du plus gros élément. 
Le drainage peut être facilité en disposant des bandes de papier filtre le long de l'éprouvette.

Lorsque le drainage est ouvert, une éprouvette saturée a la possibilite d'expulser ou d'absorber de l'eau interstitielle et donc de subir des modifications de volume.

Lorsque le sol est saturé, on peut considérer que l'essai se fait à volume constant.

La variation de volume due à la deformation du dispositif piézométrique est généralement inférieure à $0,1 \mathrm{~mm}^{3}$ pour $1 \mathrm{MPa}$.

A drainage fermé, pour une éprouvette saturée, toute variation $\Delta \sigma_{3}$ de la pression hydrostatique doit se traduire instantanément par une variation $\Delta_{u}=\Delta \sigma_{3}$ de la pression interstitielle. Les mesures sont très rapidement faussées dès que l'éprouvette ou le dispositif d'essai contiennent de l'air occlus. Afin d'éviter cet inconvénient, il est fortement conseillé d'opérer sous "contre-pression». On peut, pour cela, se reporter au mode opératoire MSL4 du LCPC.

\subsection{Conditions d'essai}

\subsubsection{Définition des essais đrainé et non drainé}

\subsubsection{Essai drainé}

Les faces inférieure et supérieure de l'éprouvette sont mises en communication avec l'extérieur par l'intermédiaire de bases poreuses saturées et de tubulures remplies d'eau.

Un robinet permet de fermer ou d'ouvrir ces tubulures.

Le système de drainage étant mis en communication avec l'atmosphère et la vitesse d'application du déviateur de contrainte étant suffisamment faible pour qu'aucune pression interstitielle ne se développe au sein de l'éprouvette, l'essai est alors dit drainé.

\subsubsection{Essai non đrainé}

Le drainage étant fermé, aucun échange avec l'extérieur n'est possible. L'essai est alors dit non drainé.

\subsubsection{Définition des essais consolidé et non consolidé}

Si, préalablement à l'application de la contrainte verticale $\sigma_{1}$, l'éprouvette est soumise à la pression hydrostatique $\sigma_{3}$, drainage ouvert jusqu'à dissipation complète des pressions interstitielles, on dit que l'essai est consolidé.

$\mathrm{Si}$, en revanche, en appliquant la pression hydrostatique $\sigma_{3}$ drainage fermé, on commence tout de suite à faire croître la contrainte verticale $\sigma_{1}$, l'essai est dit non consolidé.

\subsubsection{Mesure de la pression interstitielle "u»}

Un dispositif piézométrique à très faible variation de volume, relié à l'eau sortant de la base poreuse inférieure, permet de mesurer la pression interstitielle à drainage fermé.

Lorsque l'éprouvette est saturée, la mesure de la pression interstitielle "u» permet de déterminer les contraintes effectives définies à partir des contraintes totales :

$\sigma^{\prime}=\sigma-u$

$\tau^{\prime}=\tau$

où :

$\sigma$ : contrainte normale totale

$\sigma^{\prime}$ : contrainte normale effective

$\tau$ : contrainte tangentielle totale

$\tau^{\prime}$ : contrainte tangentielle effective 


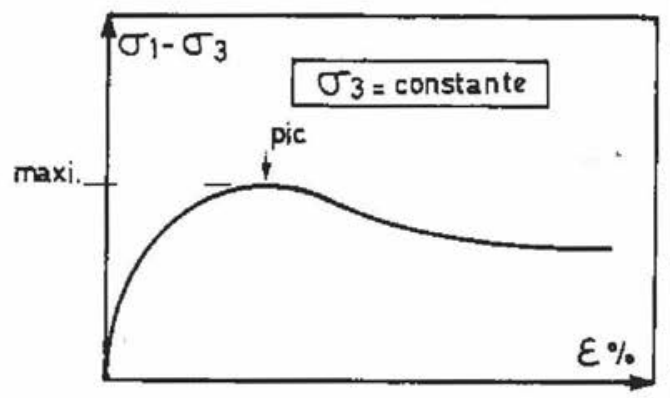

On distingue généralement :

- la rupture plane fun ou plusieurs plans de glissement),

- la rupture en tonneau.

Contrairement à la boîte de cisaillement, le plan de rupture n'est pas imposé.

D'autres représentations sont parfois utilisées, notamment dạns les pays anglo-saxons.

L'interprétation rigoureuse des essais triaxiaux est délicate, notamment dans le cas des sols non saturés et des sols surconsolidés.

Les caractéristiques $\operatorname{Cet} \varphi$ en contraintes totales ne permettent pas toujours de déterminer un critere de rupture d'une manière évidente.

\subsubsection{Différents types d'essai}

Selon les modalités de réalisation, on distingue différents types d'essai dont les plus courants sont les suivants :

UU : essai non consolidé non drainé,

$\mathrm{CU}$ : essai consolidé non drainé avec ou sans mesure de la pression interstitielle,

$\mathrm{CD}$ : essai consolidé drainé.

\subsection{Déviateur à la rupture}

Pendant I'essai, on note l'évolution du déviateur de contrainte $\sigma_{1}-\sigma_{3}$ en fonction de la déformation relative axiale $\AA$.

La valeur du déviateur à la rupture correspond conventionnellement à la valeur maximale obtenue pendant I'essai.

Lorsque la courbe déviateur-déformation relative axiale ne présente pas de maximum bien caractérisé, la valeur du déviateur «à la rupture» est, conventionnellement, celle correspondant à une déformation relative égale à $15 \%$.

On doit noter la forme de l'éprouvette au moment de la rupture et faire les corrections de section.

\subsection{Critère de rupture - Expression des résultats}

Les valeurs du déviateur à la rupture sont déterminées sur au moins trois éprouvettes du même échantillon de sol pour des étreintes latérales différentes.

Les états de contrainte à la rupture sont définis par les contraintes principales correspondantes $\sigma_{1}$ et $\sigma_{3}$.

On peut alors tracer pour chaque éprouvette un cercle de Mohr de diametre $\sigma_{1}-\sigma_{3}$ et de centre $\frac{\sigma_{1}+\sigma_{3}}{2}$ et déterminer un critère de rupture en termes de cohésion et d'angle de frottement interne.

Ce critère de rupture peut s'entendre soit en contraintes totales (court terme), soit en contraintes effectives (long terme).

Seion les modalités d'essai, on obtient les résultats suivants : 
La vérification à la rupture des fondations superficielles et des fondations profondes est généralement conduite à partir des caractéristiques apparentes déduites d'essais UU.

Le cas des sols gonflants est à examiner avec précaution.

Cet essai est une forme particulière de l'essai triaxial non consolidé, non drainé UU sans étreinte latérale.

Pour les sols fins, cette vitesse est suffisamment grande pour que l'on puisse considerer qu'il ne se produit aucun drainage pendant l'essai.

Bien que les résu/tats de cet essai soient relativement disperses, il est souhaitable de faire une correction de section droite.

\begin{tabular}{|l|c|r}
\hline $\begin{array}{l}\text { Type } \\
\text { d'essai }\end{array}$ & $\begin{array}{c}\text { Détermination } \\
\text { des caractéristiques }\end{array}$ & $\begin{array}{r}\text { Symboles } \\
\text { UU }\end{array}$ apparentes \\
\hline CU & $\begin{array}{r}\varphi_{\text {uu }} \\
\text { Cuu }\end{array}$ \\
$\begin{array}{l}\text { sans mesure } \\
\text { de u }\end{array}$ & $\begin{array}{c}\text { totales } \\
\text { cu }\end{array}$ \\
\hline CU & effectives \\
avec mesure & calculées & $\varphi^{\prime}$ \\
de u & effectives & $C^{\prime}$ \\
\hline CD & & $\varphi^{\prime}$ \\
\hline
\end{tabular}

\section{L'essai de compression simple}

\subsection{Principe de l'essai}

L'éprouvette cylindrique, généralement de section circulaire, d'élancement égal à 2, est placée entre les deux plateaux d'une presse.

Elle est soumise, jusqu'à rupture, à une charge axiale croissante appliquée à vitesse de déformation constante.

\subsection{Vitesse d'essai}

La vitesse d'application de la charge axiale est de $\uparrow, 75 \pm 0,25 \mathrm{~mm} /$ minute.

\subsection{Résistance à la compression simple $q_{u}$}

La résistance à la compression simple $q_{u}$ est, conventionnellement, le rapport de la charge axiale maximale à la section droite de l'éprouvette.

Si la charge axiale appliquée ne présente pas la valeur maximale avant un raccourcissement relatif de l'éprouvette de $15 \%$, c'est la charge correspondant à ce raccourcissement qui est prïse en compte pour la détermination de $q_{u}$.

Si une correction de section droite est faite, elle doit clairement être notée dans l'énoncé des résultats de l'essai. 


\section{C. essais de compressibilité}

II existe d'autres types d'essal de compressibilité pouvant être appliqués a des échantillons non satures, drainés axialement ou autrement, sans possibilité de déformation latérale.

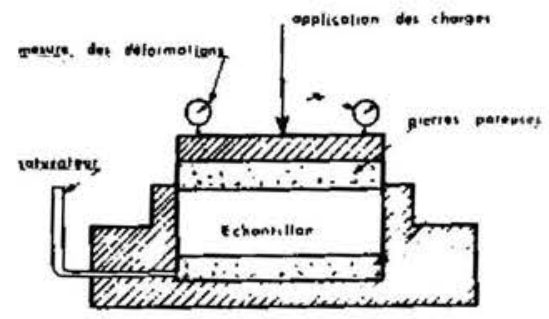

Schéma du moule adométrique

Le diametre de l'eprouvette est com. pris entre 50 et $80 \mathrm{~mm}$ et son épaisseur entre 12 et $25 \mathrm{~mm}$ suivant l'appareil utilisé.

\section{Définitions}

Les essais de compressibilité ont pour objet de mesurer les variations de volume d'un échantillon de sol placé dans un champ de contraintes uniforme avec possibilité de drainage.

Lorsque l'échantillon de sol intact est saturé, que les déformations latérales sont empéchées et que le drainage n'est possible que par les faces inférieure et supérieure de l'échantillon, l'essai de compressibilité est dit «œdométrique».

\section{L'essai œdométrique}

\subsection{Principe de l'essai}

L'échantillon de sol intact est placé dans une boîte cylindrique rigide de section circulaire entre deux pierres poreuses assurant son drainage. 
I/ existe pour cet essai un mode opératoire du Laboratoire Central des Ponts et Chaussées.

Ce processus n'est généralement pas adapté à l'étude du gonflement des sols.

La durée de chaque palier est telle que ia consolidation primaire soit terminée. Cette consolidation est généralement atteinte avant 24 heures pour des échantillons dont l'épaisseur est inférieure ou égale à $24 \mathrm{~mm}$.

On peut inclure dans la phase de chargement un cycle déchargementchargement au voisinage de la contrainte verticale initiale subie par l'échantillon dans le sol avant de poursuivre l'essai jusqu'à la contrainte finale.

Pour des essais de longue durée destinés à apprécier les caractéristiques de la consolidation secondaire, la durée de chaque palier peut atteindre 10 jours.

La charge nulle correspond en fait au poids du piston seul.
Un piston permet d'appliquer sur l'échantillon une contrainte verticale uniforme $\sigma$ constante pendant un temps déterminé.

\subsection{Processus de l'essai œedométrique}

Le processus de l'essai comprend trois phases :

- une phase de "saturation》 au cours de laquelle tout gonflement de l'échantillon est empéché,

- une phase de "chargement» au cours de laquelle est appliquée, par paliers, une contrainte $\sigma$ dont la valeur croît d'un paliẹr à l'autre en progression géométrique de raison 2 .

- une phase de "déchargement» au cours de laquelle la contrainte $\sigma$ est ramenée à une valeur nulle en passant par deux paliers intermédiaires de 24 heures ; tous les paliers de chargement et déchargement, y compris sous charge nulie, doivent être maintenus pendant une durée au moins égale à 24 heures.

\subsection{Mesure de la déformation}

La mesure de la déformation est, suivant le type de l'appareil, effectuée :

- soit avec un comparateur centré sur le piston,

- soit avec deux comparateurs diamétralement opposés sur les bords du piston. Ces comparateurs, mécaniques ou électriques, doivent être sensibles au 1/100ème de millimètre.

\subsection{Mesure de la charge d'essai}

La valeur de la charge d'essai doit être connue à $\pm 1 \%$ près. 
Un exemple de coumb de compressibilité est donné en annexe.

L'indice des vides "en de l'éprouvette de sol à l'issue d'un palier de chargement ayant engendré une déformation $\Delta h$ de cette éprouvette par rapport à sa hauteur initiale avant tout chargement peut être calculé par la formule :

$e=\frac{h-h p}{h p}$

où :

$h=h o-\Delta h$

ho étant la hauteur de l'éprouvette à'l'issue de la phase de "saturation", et :

$h p=\frac{W d}{\gamma_{S} \cdot S}$

où : $W d, \gamma_{s}$ et $S$ sont respectivement : - le poids de l'échantillon desséché après passage à l'etuve,

- le poids volumique des grains constitutifs du sol,

- la section droite de l'éprouvette.

On peut figurer ces mesures sur un graphique dit acourbe de consolidationn.

Deux exemples de "courbes de consolisationy sont donnés en annexe : l'une construite en fonction du logarithme du temps, l'autre en fonction de la racine carrée du temps.

Le module cedométrique Eœd $\left(\sigma_{n}\right.$, $\left.\sigma_{n+1}\right)$ entre deux paliers successifs de chargement est donné par la formule :

$\left.11+e_{n}\right) \frac{\sigma_{n}+1-\sigma_{n}}{e_{n}-e_{n}+1}$

\section{Expression et présentation des résultats}

\subsection{Courbe de compressibilité cedométrique}

Les résultats des mesures sont représentés sous forme d'un graphique dit "courbe de compressibilité œdométrique» en portant :

- les contraintes en abscisse selon une échelle logarithmique,

- les indices des vides correspondants en ordonnée selon une échelle arithmétique.

\subsection{Courbes de consolidation}

Les mesures de déformation effectuées au cours d'un palier de chargement déterminé sont consignées sur un tableau donnant à chaque instant de mesure la lecture correspondante des comparateurs.

\subsection{Paramètres de déformation}

Les documents précédents permettent de déterminer les valeurs des paramètres de déformation suivants :

3.3.1 Modules œdométriques Eœd $\left(\sigma_{n}, \sigma_{n+1}\right)$ entre deux paliers de chargement successifs sous les contraintes $\sigma_{\mathrm{n}}$ et $\sigma_{\mathrm{n}+1}$ auxquelles correspondent les indices de vides $e_{n}$ et $e_{n+1}$. 
La courbe e - log $\sigma$ présente souvent dans le domaine des contraintes les plus fortes une partie linéaire. $C_{c}$ est la pente de cette partie linéaire :

$$
C_{c}=\frac{\Delta e}{\Delta(\log \sigma)}
$$

3.3.2 Indice de compression $\mathrm{C}_{\mathrm{c}}$ lorsque l'allure de la coube cedométrique permet de le calculer.

3.3.3 Coefficient de consolidation verticale $C_{v}$ au cours d'un palier déterminé.

Ce coefficient se déduit de la courbe de consolidation correspondante. 


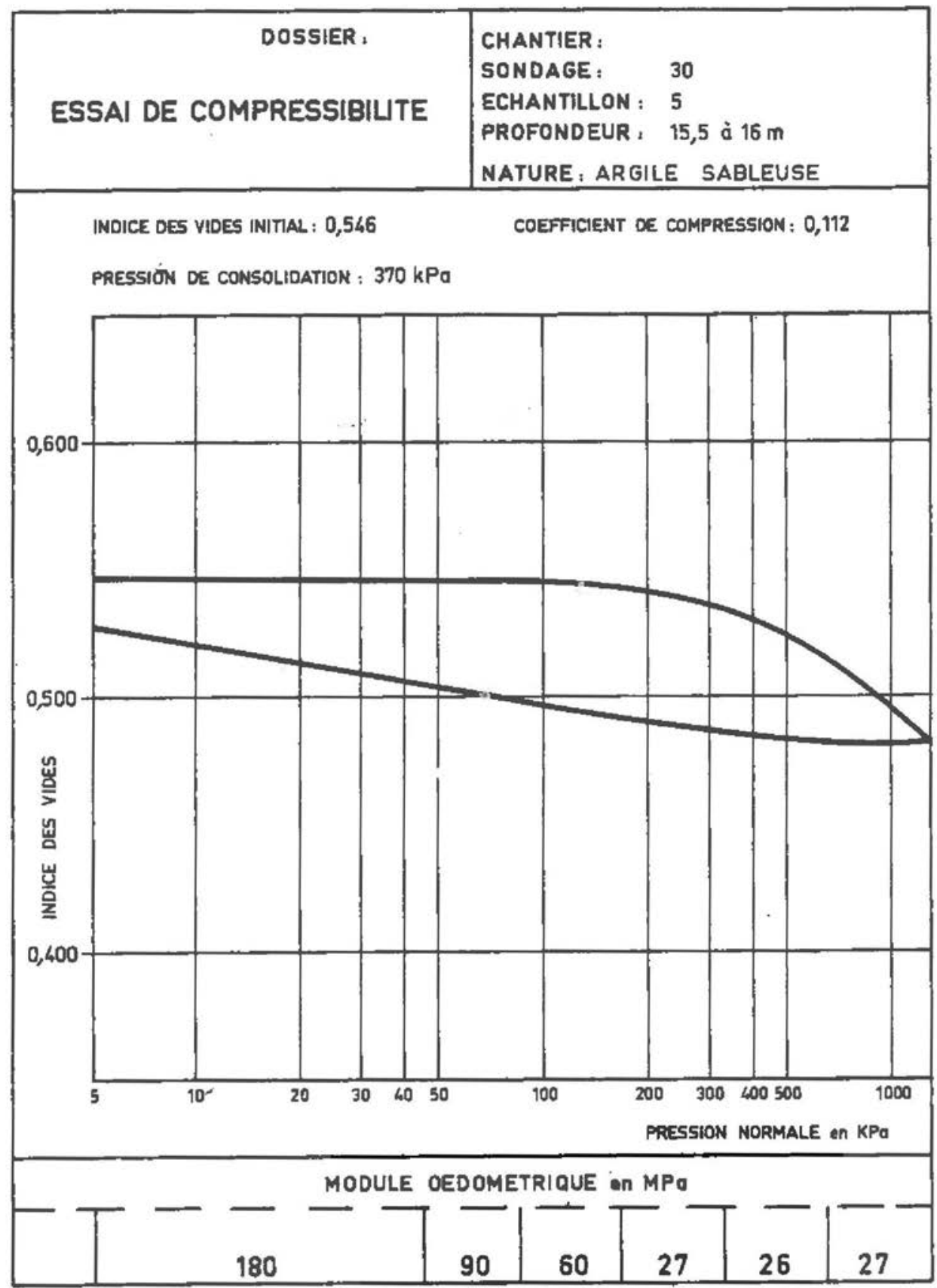



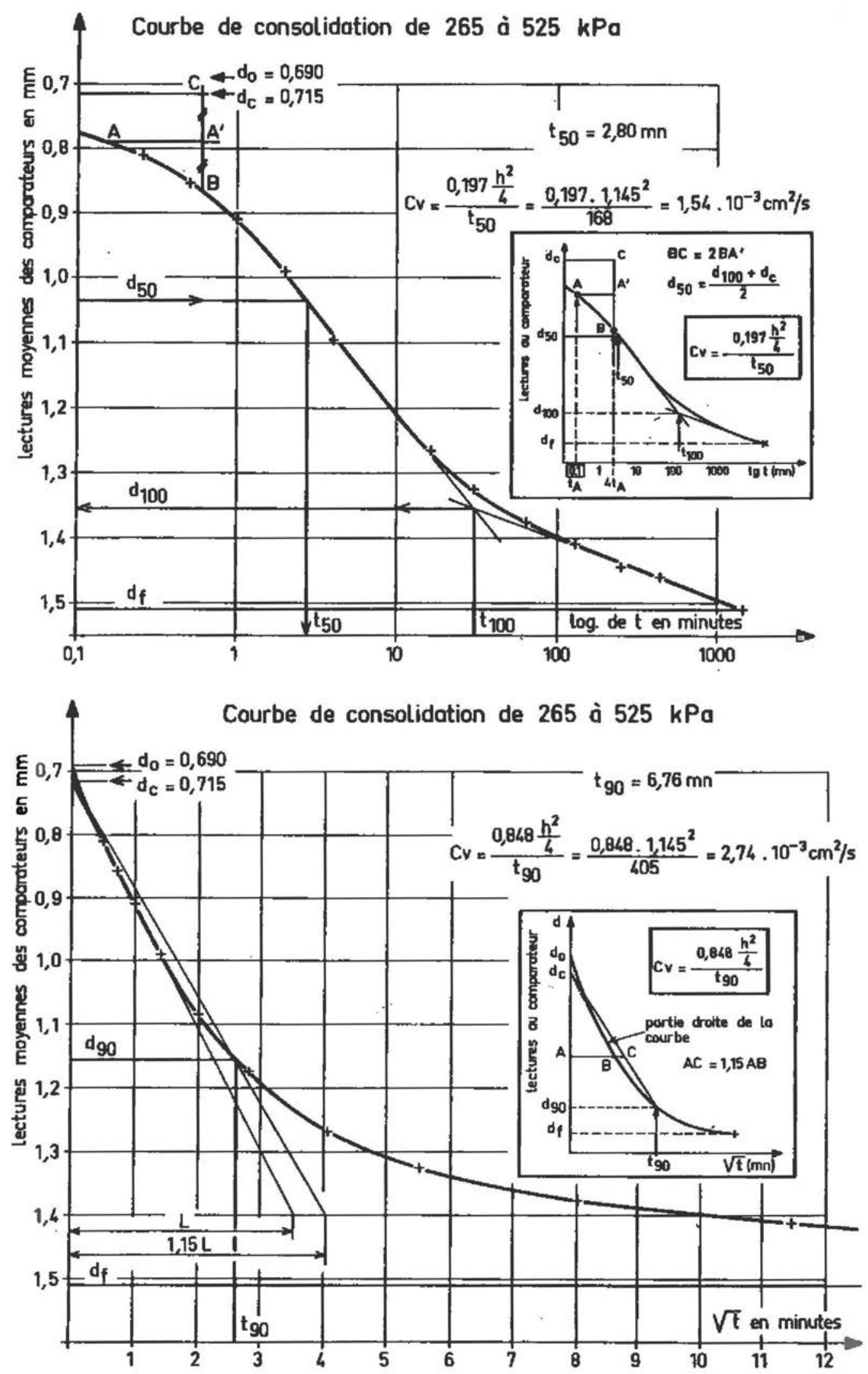


\section{D. essais de compactage}

Catte appellation fait reférence à l'ingenieur Proctor qui, le premier, a defini les modalités d'essai de compactage.

Les essais "Proctor" sont inadaptés aux sols contenant plus de $25 \%$ en poids d'eléments retenus aux tamis de $20 \mathrm{~mm}$.

\section{Définition}

Les essais de compactage sont des essais conventionnels qui ont pour but d'étudier l'influence de la teneur en eau d'un échantillon de sol sur le poids volumique sec de cet échantillon soumis à une énergie de compactage déterminée.

\section{Principe}

Un essai de compactage consiste à compacter avec une énergie définie un échantillon de sol remanié dans un mouie normalisé et à mesurer le poids volumique sec obtenu. L'essai est recommencé pour différentes teneurs en eau.

Il existe deux types d'essai d'usage courant : I'essai dit «Proctor Normal» (PN) et I'essai dit «Proctor Modifién (PM).

Ces deux essais diffèrent par l'énergie de compactage mise en cuvre.

Les prescriptions suivantes ne concernent que ces essais normalisés.

\section{Processus}

Le compactage de l'échantillon qui ne doit pas contenir d'éléments supérieurs à $20 \mathrm{~mm}$ se fait par couches d'épaisseur déterminée sous un nombre donné de coups d'une dame de caractéristiques normalisées. 
Le processus d'exécution de ces essais fait l'objet d'un mode opératoire établi par le Laboratoire Central des Ponts et Chaussées.

L'utilisation de systèmes automatiques de damage est recommandée.

Lorsque l'échantillon de sol ne comporte pas d'éléments de dimensions supérieures à $5 \mathrm{~mm}$, ces essais peuvent etre effectués dans un moule normalise Proctor de $10,15 \mathrm{~cm}$ de diametre intérieur et de $11,7 \mathrm{~cm}$ de hauteur.

Chaque couche est alors compactee par 25 coups de dame.

Un exemple de feuille d'essai est donné en annexe.
Le moule utilisé est le moule normalisé CBR de $15,2 \mathrm{~cm}$ de diamètre intérieur et de $15,2 \mathrm{~cm}$ de hauteur. Chaque couche est compactée par 55 coups de dame.

Pour I'essai Proctor «Normal», I'échantillon de sol est compacté en 3 couches d'égales épaisseurs à l'aide d'une dame de 2,44 daN tombant librement d'une hauteur de $30,4 \mathrm{~cm}$.

Pour l'essai Proctor "Modifié», l'échantillon de sol est compactế en 5 couches d'égales épaisseurs à l'aide d'une dame de 4,45 daN tombant librement d'une hauteur de $45,7 \mathrm{~cm}$.

\section{Présentation des résultats}

Pour un même sol, les essais Proctor donnent des couples de valeur teneur en eau-poids volumique sec.

On porte sur un graphique en échelle arithmétique les points représentatifs de chacun des couples :

- en ordonnée : le poids volumique sec,

- en abscisse : la teneur en eau.

La courbe passant par les points représentatifs présente un maximum dit "Optimum Proctor» normal ou modifié selon la nature de l'essai. Ce maximum définit la teneur en eau $w_{\text {opt }}$ et le poids volumique $\gamma_{d}$ max.

Cette courbe doit comporter au moins 5 points. La feuille d'essai doit clairement porter l'indication de la nature de l'essai exécuté, ainsi que celle du moule utilisé. 


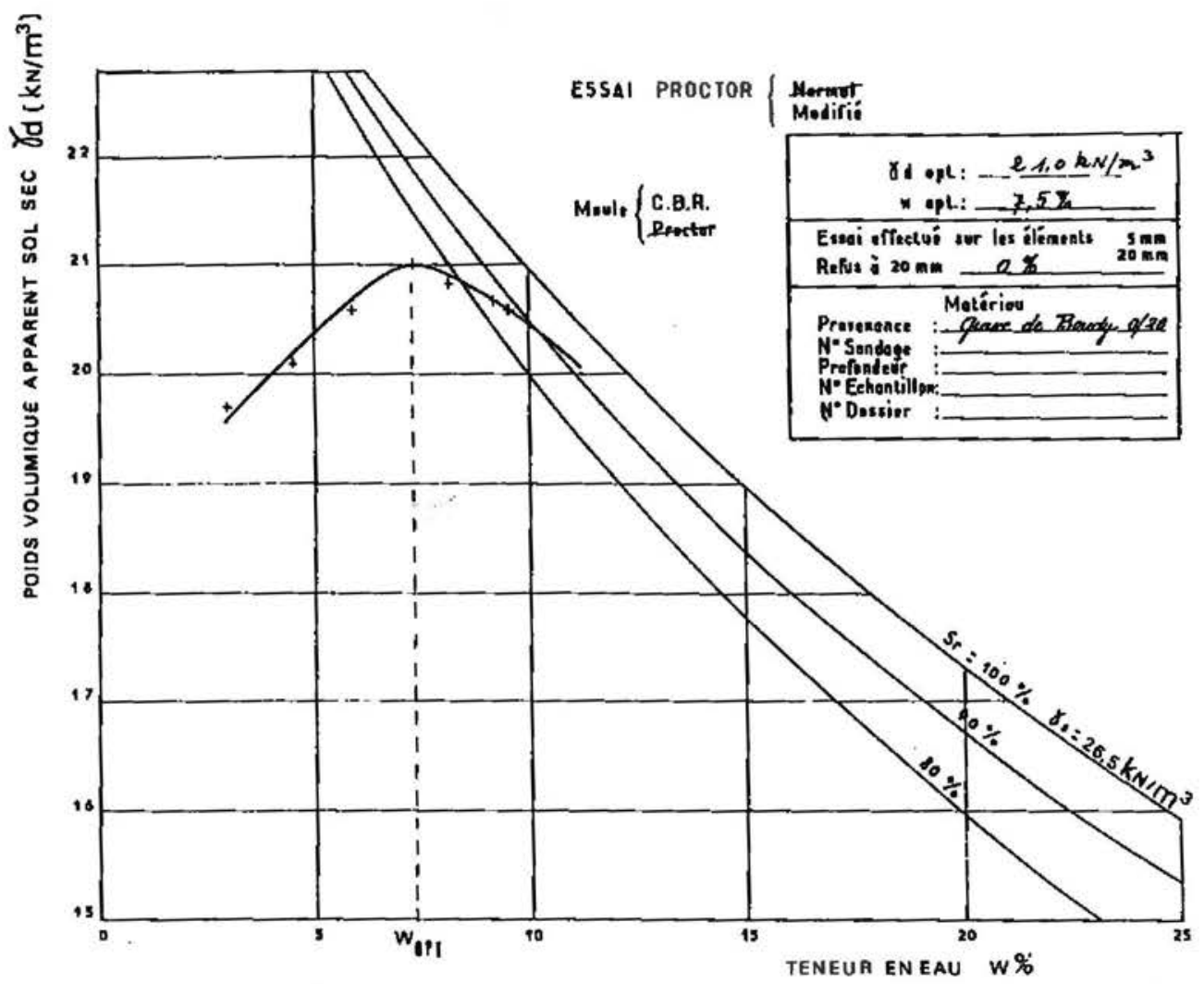




\section{E. essai de poinçonnement CBR}

Cet essai conventionnel, développé par I'Administration des Ponts et Chaussées de l'Etat de Californie, est uniquement utilisé pour la détermination des structures de chaussées souples. Les résultats donnés par cet essai ne sont pas représentatifs des propriêtés mécaniques de l'échantillon de sol étudié.

L.e mode opératoire original est celui décrit par I'US Corps of Engineers dans le document suivant : aEngineering Manual of Military Construction", Part XII, Chap. 2, "Airfield Pavement Design, Flexible Pavement", July 1951.

Cet essai n'est pas adapté aux sols contenant des éléments de dimensions superieures aे $20 \mathrm{~mm}$.

On peut également réaliser des essais de poinçonnement sur des échantillons de sol compactés avec des énergies différentes ou à des teneurs en eau quelconques avec ou sans imbibition prealable : ces essais ne permettent pas de déterminer l'indice

\section{Définition}

L'essai CBR est un essai de poinçonnement réalisé sur un échantillon de sol préalablement mis en place dans des conditions de compactage et d'hydratation déterminées dans un moule normalisé à l'aide d'un mandrin de forme cylindrique et de section circulaire enfoncé verticalement dans l'échantillon.

\section{Processus}

L'échantillon de sol, hydraté à une teneur en eau correspondant à $\pm 0,5 \%$ près à la teneur en eau optimale déduite d'un essai «Proctor Modifié» préalablement réalisé sur le même sol, est compacté dans le moule "CBR» déjà décrit selon le mode opératoire correspondant à l'essai "Proctor Modifié».

On vérifie alors que son poids volumique sec correspond bien au poids volumique'sec optimal obtenu à I'issue de r'essai Proctor Modifié préalable. Puis, l'ensemble moule-échantillon convenablement équipé d'un dispositif permettant la mesure de la déformation axiale de l'échantillon et d'une surcharge annulaire appropriée correspondant au poids qu'exerceront 
portant californien (CBR). Leur usage est donc réservé à certaines études très particulières.
Un exemple de graphique représentatif est donné en annexe. les couches supérieures de terrain sur le sol étudié au moins égale à $4,45^{\circ}$ daN, est noyé verticalement dans un bac à imbibition rempli d'eau.

Après 4 jours d'imbibition (à \pm 1 heure près), la déformation axiale de l'échantillon est mesurée. L'ensemble moule-échantillon est mis à égoutter pendant 15 minutes puis pesé pour déterminer la teneur en eau de l'échantillon. Puis, on recharge l'échantilІол à l'aide d'une première surcharge annulaire de 2,22 daN et on réalise le contact franc de l'échantillon avec le mandrin cylindrique de $4,96 \mathrm{~cm}$ de diamètre.

On complète le chargement annulaire de l'échantillon a l'aide d'une autre surcharge dont le poids doit compléter celui de la première de manière à atteindre la valeur de la surcharge utilisée pendant l'imbibition. Puis on procède au poinçonnement de l'échantillon en maintenant la vitesse d'enfoncement du mandrin constante et égale à $0,127 \mathrm{~cm} /$ minute.

Le déplacement du mandrin est mesuré à l'aide d'un comparateur au 1/100éme de millimètre et la contrainte appliquée sur ce mandrin mesurée à 0,05 $\mathrm{MPa}$ près.

Les mesures correspondantes sont notées pour les enfoncements suivants du mandrin : $0,5,1,2,3 . .10$ millimètres. L'essai est arrêté lorsque l'enfoncement de 10 millimètres est obtenu.

On détermine alors la teneur en eau de l'échantillon au voisinage de l'empreinte laissée par le mandrin.

\section{Représentation des résultats obtenus}

Les mesures faites en cours d'essai sont reportées sur un graphique représentant en échelles arithmétiques la variation de l'enfoncement du mandrin en fonction de la charge appliquée à la base du mandrin sur l'échantillon. Cette courbe présente généralement une concavité régulière dirigée vers l'axe des enfoncements.

Si la courbe obtenue présente une inflexion au voisinage de l'origine, la partie correspondante de la coube doit être remplacée par la tangente au point d'inflexion. Le point de concours de cette tangente avec l'axe des enfoncements sera pris comme origine des abscisses pour le calcul de l'Indice Portant.

\section{Expression des résultats}

Sur la courbe représentative, on lit la contrainte $\sigma 2,54$ correspondant à un enfoncement de $2,54 \mathrm{~mm}$ 
L'indice portant californien (CBR) correspond dans la définition d'origine au rapport de la resistance au poinçonnement obtenue sur le sol étudié a celle d'un sol étalon constitué par une grave concassée. et celle correspondant à un enfoncement de $5,08 \mathrm{~mm}$ $(05,08)$.

L'Indice Portant Californien (CBR) est par définition la valeur du rapport :

\section{$\sigma 2,54 \mathrm{MPa}$}

$6,86 \mathrm{MPa}$

et ce à condition que le rapport :

$\underline{\sigma 5,08 \mathrm{MPa}}$

$10,29 \mathrm{MPa}$

ne lui soit pas supérieur.

Si ce deuxième rapport est plus grand que le premier, I'essai doit être recommencé.

A I'issue de ce nouvel essai, on prend pour Indice Portant Californien la valeur du rapport $\frac{\sigma 5,08}{10,29}$ si ce rapport a une valeur très voisine de celle obtenue au cours du premier essai. 


\section{ESSAI GBR}

\section{ECHANTILLON N}

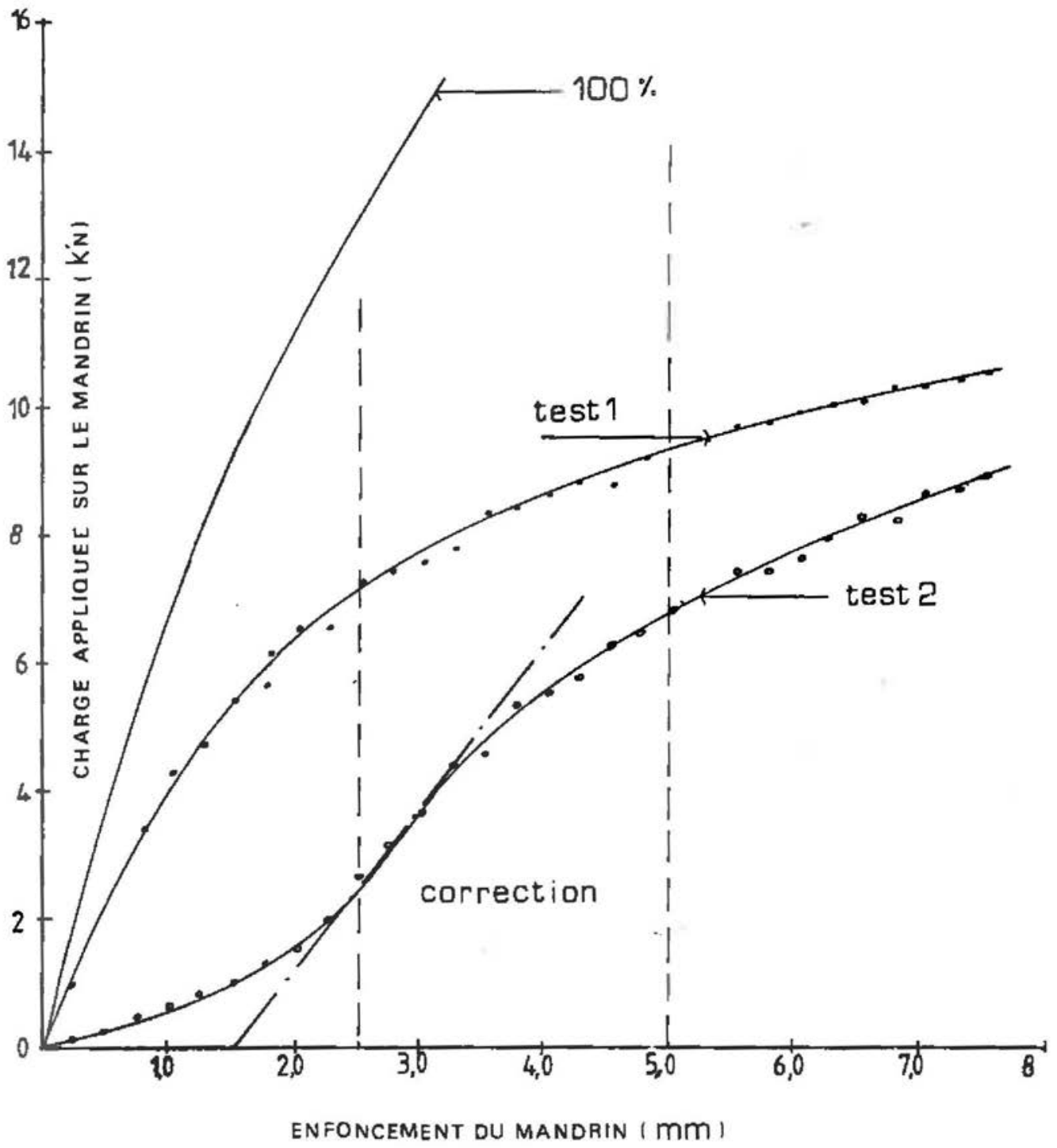




\section{F. essais cycliques et dynamiques}

Ces essais ont été développés pour étudier le comportement des sols soumis a des sollicitations cycliques et dynamiques du type de celles provoquées par les séismes et les machines vibrantes.

Ces caracteristiques sont mesurées dans une plage de distorsion déterminée allant généralement de $10^{-5}$ aे $10^{-2}$ environ.
On peut se reporter sur ce point aux publications spécialisées telles que : - "Dynamic Geotechnical Testing", ASTM - STP 654,

ou :

- "Joumal of Soil Mechanics and Foundation Division» de l'ASCE.

\section{Définition}

Les essais cycliques et dynamiques ont essentiellement pour objet la mesure des caractéristiques cycliques et dynamiques des sols et l'étude de la variation de leur résistance et de leur. déformabilité en fonction du mode d'application des sollicitations.

\section{Caractéristiques mesurées}

Les principales caractéristiques cycliques et dynamiques dont on recherche la détermination au cours de ces essais sont les suivantes :

- le module d'élasticité cyclique,

- le module de cisaillement cyclique,

- le taux d'amortissement critique,

- l'évolution des pressions interstitielles.

3 Principaux types d'essais cycliques et dynamiques

Les principaux types d'essais cycliques et dynamiques sur éprouvettes sont les suivants :

- I'essai cyclique à I'appareil triaxial,

- I'essai cyclique à la boîte de cisaillement à parois latérales mobiles,

- I'essai cyclique de cisailiement par torsion,

- I'essai à la table vibrante,

- r'essai à la colonne résonnante.

\section{Processus d'essais}

Les processus de ces essais dont l'usage est loin d'être généralisé ne sont pas encore normalisés.

Le laboratoire d'essai doit préciser dans son compte rendu le mode de préparation des éprouvettes, le processus d'essai appliqué depuis le début jusqu'à la fin de l'essai et la précision des mesures faites. 


\section{G. essais spécifiques}

\section{sur les matériaux rocheux}

Le comportement des matériaux rocheux dépend pour une tres large part de la fissuration et de l'anisotropie de ces matériaux dues à l'existence de discontinuités qui constituent des surfaces de rupture privilégiées.

La faible déformabilite de la matrice de ces matériaux et sa forte cohésion impliquent l'application de champs de contraintes beaucoup plus éleveses que pour les sols courants.

\section{Définition}

On entend ici par "essais spécifiques» sur les matériaux rocheux des essais mécaniques qui nécessitent. I'utilisation d'appareillages très différents de ceux décrits précédemment pour les sols, tant par l'amplitude des contraintes appliquées sur les échantillons que par la nature même des paramètres mesurés.

\section{Principaux paramètres mesurés}

Outre les identifications pétrographiques et les paramètres habituels tels que :

- teneur en eau,

- porosité,

- poids volumique,

les essais spécifiques dont il est question ici ont pour but de mesurer :

- la résistance à la compression et à la traction uniaxiales dans des directions données,

- la résistance à la compression dans des conditions triaxiales,

- la résistance au cisaillemenț selon un plan de discontinuité déterminé,

- la déformabilité selon le champ de contraintes appliqué, 
"Rock characterization, testing and monitoring", ISRM suggested methods, dernière édition parue en février 1981 chez Pergamon Press à Oxford (Grande-Bretagne).

Une description de ces essais et certains modes opératoires figurent également en français dans louvrage - La mécanique des roches appliquée aux ouvrages du génie civily publié en 1976 par I'AAIAE et I'ENPC.
- la continuité (degré de fissuration) à l'aide de la cétérité des ondes,

- l'effet d'échelle,

- l'abrasivité,

- etc.

\section{Processus d'essais}

Les processus des essais spécifiques utilisés en mécanique des roches font l'objet d'une publication régulièrement mise à jour par la Société Internationale de Mécanique des Roches. 


\section{ETUDE GEOTECHNIQUE}

\section{ET -}

\section{RECONNAISSANCE DES SOLS}

Ce document dorine aux Mairres d' ouvrage, bux Maitres d'auvre et aux Concepteurs lopportunite de mizux apmecier la diversite des movens actuellement mis a leur disposition pour pavenir a une meillaure definition des projess de fon detion.

Il decrit de facon precise les dibfereates methodes de sondages at de prelevenients de sols effectues lars de la recotinaissanea geotechnique ains que des essals geo: physigues menes pendant l etude préalable.

Cet stivrage rassemble pour la premiere fois la deseription des "esscis de sols" donit les modes operatoires existent de tacon éparse i essaits de laboratoirit essai de plaques, essais pressientietrique, penetrometrique, scissometrique, essais d'eau. 